\title{
Precisely Encoding Geometric Features into Discrete Linear Polymer Chains for Robust Structure Engineering
}

\author{
Dongdong Zhou, ${ }^{1,2}$ Miao Xu, ${ }^{1}$ Zhuang Ma, ${ }^{1}$ Zhanhui Gan, ${ }^{1}$ Rui Tan, ${ }^{1,2}$ Shuai Wang, ${ }^{1}$ Zhengbiao Zhang, ${ }^{3}$ \\ and Xue-Hui Dong ${ }^{1,2 *}$
}

${ }^{1}$ South China Advanced Institute for Soft Matter Science and Technology, School of Molecular Science and Engineering, South China University of Technology, Guangzhou 510640, China

${ }^{2}$ Guangdong Provincial Key Laboratory of Functional and Intelligent Hybrid Materials and Devices, South China University of Technology, Guangzhou 510640, China

${ }^{3}$ College of Chemistry, Chemical Engineering and Materials Science, Soochow University, Suzhou 215123, China

*Corresponding author: xdong@scut.edu.cn

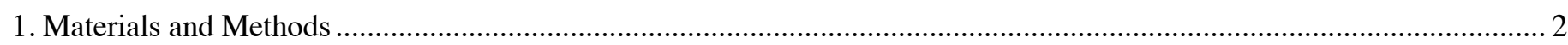

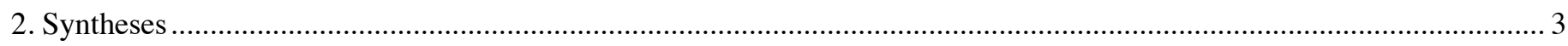

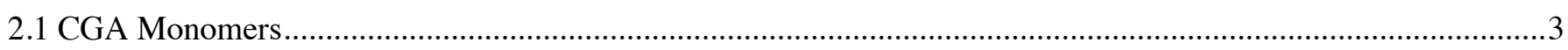

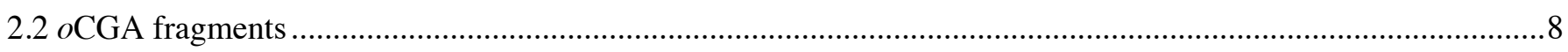

2.3 Discrete macromolecules with diverse geometries......................................................................... 13

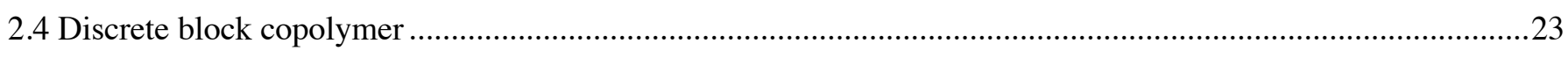

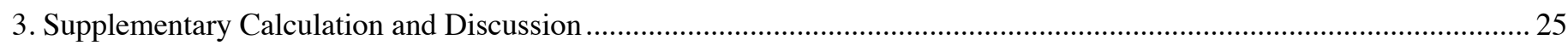

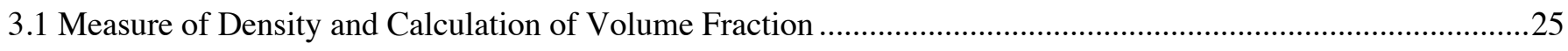

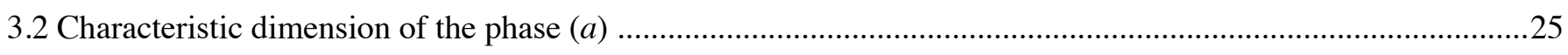

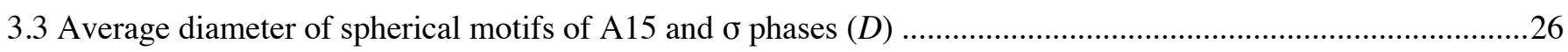

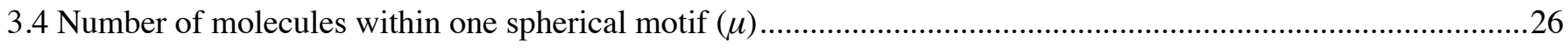

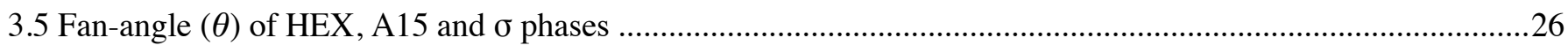

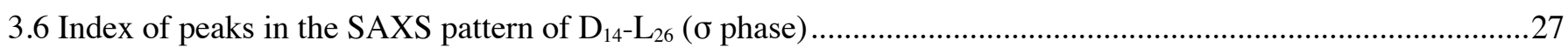

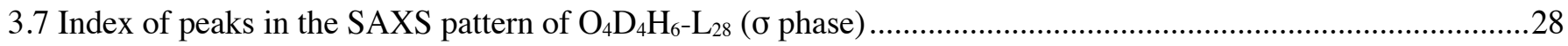

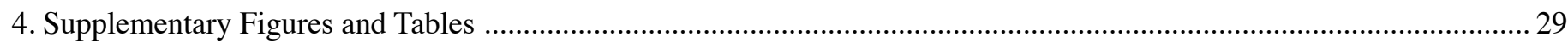




\section{Materials and Methods}

The following chemicals are used as received: D,L-glutamic acid (Adamas, > 98\%), 1-hexadecanol (Greagent, > 98\%), 1-dodecanol (Adamas, > 98\%), 1-octanol (Adamas, > 98\%), 1-butanol (Adamas, > 98\%), 1,4-dioxane (Greagent, > 99.7\%), acetic acid (Greagent, > 99.5\%), benzyl bromide (BnBr, Adamas, 99\%),

potassium carbonate $\left(\mathrm{K}_{2} \mathrm{CO}_{3}\right.$, Greagent, $\left.99.5 \%\right)$, cesium carbonate $\left(\mathrm{Cs}_{2} \mathrm{CO}_{3}\right.$, Adamas, $\left.99.9 \%\right)$, tertbutyldimethylsilyl chloride (TBDMSCl, TCI, 98\%), imidazole (TCI, 99\%), Pd/C (10 wt\%, Aldrich), boron trifluoride etherate $\left(\mathrm{BF}_{3}-\mathrm{Et}_{2} \mathrm{O}, \mathrm{TCI}, 98 \%\right), \mathrm{N}, \mathrm{N}$-diisopropylcarbodiimide (DIC, Adamas, 98\%), Dcamphorsulfonic acid (TCI, 99\%), benzyl alcohol (Bn, TCI, 99\%). Anhydrous solvents, including toluene, dimethylformamide (DMF), and dichloromethane $\left(\mathrm{CH}_{2} \mathrm{Cl}_{2}\right)$, were obtained with an INERT Pure Solv System (Inert Corporation, USA). 4-(Dimethylamino) pyridinium-4-toluenesulfonate (DPTS) and discrete $o$ LA ( $\mathrm{L}_{m}$, $m=24,26,28,30$, and 32 ) were synthesized according to literature. ${ }^{1}$

Automated column chromatography was conducted on a SepaBean ${ }^{\mathrm{TM}}$ machine $\mathrm{T}$ (SanTai Technologies, China) with an automated variable wavelength UV-VIS detector $(200-400 \mathrm{~nm})$.

${ }^{1} \mathrm{H}$ and ${ }^{13} \mathrm{C}$ NMR spectra were recorded on Bruker $500 \mathrm{MHz}$ spectrometers using $\mathrm{CDCl}_{3}(\mathrm{Cambridge})$ as deuterated solvent. The spectra were referenced to the residual proton impurities in the $\mathrm{CDCl}_{3}$ at $\mathrm{d} 7.26 \mathrm{ppm}$.

Size exclusion chromatography (SEC) analyses were conducted on a Tosoh HLC-8320 instrument equipped with three TSKgel columns (SuperH2000, SuperH3000, and SuperH4000) in series, a double flow type RI detector, and a UV-8320 UV detector, under an eluent flow rate of $0.6 \mathrm{~mL} / \mathrm{min}$ (THF). Regular SEC calibrations were conducted with polystyrene standards (Polymer Laboratories).

Matrix-assisted laser desorption ionization time-of-flight (MALDI-ToF) mass spectra (MS) were acquired on an UltrafleXtreme MALDI-ToF mass spectrometer (Bruker Daltonics, Germany) equipped with an Nd:YAG smart beam-II laser with 355-nm wavelength and $200 \mathrm{~Hz}$ firing rate using trans-2-[3-(4-tert-butylphenyl)-2methyl-2-propenylidene]-malononitrile (DCBT, Aldrich, > 98\%) as matrix. The cationizing agent sodium trifluoroacetate was prepared in ethanol at a concentration of $10 \mathrm{mg} / \mathrm{mL}$. The matrix and cationizing salt solutions were mixed in a ratio of $10 / 1(\mathrm{v} / \mathrm{v})$. The instrument was calibrated prior to each measurement with external PMMA at the molecular weight under consideration. All samples were dissolved in THF at a concentration of $10 \mathrm{mg} / \mathrm{mL}$.

Small angle X-ray scattering (SAXS) was performed on Shanghai Synchrotron Radiation (SSRF), beamline BL16B1. The incident X-ray wavelength $(\lambda)$ is $0.124 \mathrm{~nm}$ (photon energy: $10 \mathrm{keV}$; photo flux: $1 \times 10^{11} \mathrm{phs} / \mathrm{s}$ ). The beam size is less than $0.4 \times 0.5 \mathrm{~mm}^{2}$. Scattered X-rays were captured on a 2-dimensional Pilatus detector. 
The instrument was calibrated with diffraction patterns from silver behenate. Samples were heated to $80{ }^{\circ} \mathrm{C}$ for 30 min on a Linkam hot stage to erase thermal history, and then annealed between 35 and $40{ }^{\circ} \mathrm{C}$ for $2 \mathrm{~h}$ (35 ${ }^{\circ} \mathrm{C}$ for $\mathrm{O}_{4} \mathrm{D}_{4} \mathrm{H}_{4}-\mathrm{L}_{32}$ and $\mathrm{H}_{4} \mathrm{D}_{6} \mathrm{O}_{4}-\mathrm{L}_{m}, m=26,28,30,32 ; 38{ }^{\circ} \mathrm{C}$ for $\mathrm{O}_{4} \mathrm{D}_{6} \mathrm{H}_{4}-\mathrm{L}_{28}$; the rest at $40{ }^{\circ} \mathrm{C}$ ). The resultant DQC phase was further annealed at elevated temperatures, which prompts transition to stable FrankKasper phases. In-situ experiments were carried out with a Linkam hot stage mounted onto the SAXS apparatus in BL16B1. Samples were sealed with aluminum foil for good thermal conductivity. The heating rate was $10{ }^{\circ} \mathrm{C} / \mathrm{min}$. Samples were equilibrated for 3 minutes at each temperature before collecting data.

\section{Syntheses}

\subsection{CGA Monomers}

\subsubsection{BGA}

Synthesis of $\mathrm{H}_{2} \underline{\mathrm{N}-\mathrm{BGA}-\mathrm{COOH}}$. L-glutamic acid (50.0 g, $\left.339.83 \mathrm{mmol}\right)$ was suspended in 1-butanol (150 mL). Concentrated $\mathrm{H}_{2} \mathrm{SO}_{4}(95 \%, 20.0 \mathrm{~mL})$ is added dropwise under vigorously stirring at room temperature. After reacting overnight, triethylamine $(20.0 \mathrm{~mL})$ was added dropwise to the mixture. The reaction was then diluted with water $(70 \mathrm{~mL})$ and methanol $(130 \mathrm{~mL})$. Additional triethylamine $(80.0 \mathrm{~mL})$ was added, and the mixture was further stirred for 30 minutes. The resultant precipitate was filtered out and recrystallized from ethanol/deionized water $(500 \mathrm{~mL}$, volume ratio 3/1). The obtained product was washed by water and methanol, and dried at $25{ }^{\circ} \mathrm{C}$ in vacuo $(38.0 \mathrm{~g}, 55 \%$ yield $)$.

Synthesis of HO-BGA-COOH. $\mathrm{H}_{2} \mathrm{~N}-\mathrm{BGA}-\mathrm{COOH}(30.00 \mathrm{~g}, 147.61 \mathrm{mmol})$ was suspended in a mixture of acetic acid and deionized water $(300 \mathrm{~mL}$, volume ratio $1 / 3)$. The mixture was heated to $60{ }^{\circ} \mathrm{C}$, and tert-butyl nitrite $(30.0 \mathrm{~mL}, 250.19 \mathrm{mmol})$ was added dropwise under stirring for $120 \mathrm{~min}$. The reaction solution was diluted by deionized water $(300 \mathrm{~mL})$ and extracted by ethyl acetate $(200 \mathrm{~mL})$ three times. The organic layers were collected, combined, and extracted by deionized water. The solvent was removed by rotary evaporation, and the obtained product was used directly without further purification.

Synthesis of HO-BGA. HO-BGA-COOH (30.00 g, $146.90 \mathrm{mmol})$ was dissolved in DMF (300 mL) and treated with $\mathrm{K}_{2} \mathrm{CO}_{3}(10.14 \mathrm{~g}, 73.45 \mathrm{mmol})$ for $1 \mathrm{~h}$ at room temperature. Benzyl bromide $(30.14 \mathrm{~g}, 176.28 \mathrm{mmol})$ was added and stirred overnight at room temperature. The reaction was then diluted by deionized water $(300 \mathrm{~mL})$ and extracted with ethyl acetate $(\mathrm{EtOAc}, 3 \times 200 \mathrm{~mL})$. The combined organic layers were further washed with deionized water $(3 \times 100 \mathrm{~mL})$ and dried with $\mathrm{Na}_{2} \mathrm{SO}_{4}$. After removal of the solvent in vacuo, the crude material was purified by automated column chromatography using hexane/EtOAc (gradient 100/0 to 90/10) as eluent. The pure product was obtained as a colorless oil (26.20 g, 61\% yield). ${ }^{1} \mathrm{H}-\mathrm{NMR}\left(500 \mathrm{MHz}, \mathrm{CDCl}_{3}, \delta\right): 7.40-$ 
$7.30(5 \mathrm{H}, \mathrm{Ar}-\underline{H}), 5.21\left(2 \mathrm{H}, \mathrm{Ar}-\underline{\mathrm{C}}_{2} \mathrm{O}\right), 4.23\left(1 \mathrm{H}, \mathrm{HOC} \underline{H}\left(\mathrm{CH}_{2}\right) \mathrm{CO}\right), 4.05\left(2 \mathrm{H}, \mathrm{OC}_{2} \mathrm{CH}_{2} \mathrm{CH}_{2}\right), 2.45(2 \mathrm{H}$, $\left.\mathrm{CHCH}_{2} \underline{\mathrm{H}}_{2} \mathrm{CO}\right), 2.18\left(1 \mathrm{H}, \mathrm{CHC} \underline{H}_{2} \mathrm{CH}_{2} \mathrm{CO}\right), 1.95\left(1 \mathrm{H}, \mathrm{CHC}_{2} \mathrm{CH}_{2} \mathrm{CO}\right), 1.60\left(2 \mathrm{H}, \mathrm{OCH}_{2} \underline{\mathrm{H}}_{2} \mathrm{CH}_{2} \mathrm{CH}_{2}\right), 1.35$ $\left(2 \mathrm{H}, \mathrm{OCH}_{2} \mathrm{CH}_{2} \mathrm{CH}_{2} \mathrm{CH}_{3}\right), 0.88\left(\mathrm{CH}_{2} \mathrm{CH}_{2} \mathrm{CH}_{3}\right)$.

Synthesis of BGA. HO-BGA-Bn (15.00 g, $50.96 \mathrm{mmol})$ and imidazole $(8.66 \mathrm{~g}, 127.40 \mathrm{mmol})$ were dissolved in dried DMF $(150 \mathrm{~mL})$ in a $250 \mathrm{~mL}$ round bottom flask under argon atmosphere. Tertbutyldimethylsilyl chloride (TBDMSCl, $15.36 \mathrm{~g}, 101.92 \mathrm{mmol}$ ) were then added and stirred at room temperature overnight. The mixture was quenched by saturated $\mathrm{NaHCO}_{3}(200 \mathrm{~mL})$ and extracted with EtOAc $(3 \times 200 \mathrm{~mL})$. The combined organic layers were dried over $\mathrm{Na}_{2} \mathrm{SO}_{4}$ and the solvent was removed, giving crude product as colorless oil. The crude material was purified by automated column chromatography using hexane/EtOAc (gradient 100/0 to $95 / 5)$ as eluent. Pure product was obtained as a colorless oil (19.78 g, 95\% yield). ${ }^{1} \mathrm{H}-\mathrm{NMR}(500 \mathrm{MHz}$, $\left.\mathrm{CDCl}_{3}, \delta\right)$ : 7.40-7.30 (5H, Ar- $\left.\underline{H}\right), 5.21\left(2 \mathrm{H}, \mathrm{Ar}-\mathrm{C} \underline{H_{2} \mathrm{O}}\right), 4.30\left(1 \mathrm{H}, \mathrm{HOC} \underline{H}\left(\mathrm{CH}_{2}\right) \mathrm{CO}\right), 4.03\left(2 \mathrm{H}, \mathrm{OC}_{2} \mathrm{CH}_{2} \mathrm{CH}_{2}\right)$, $2.45\left(2 \mathrm{H}, \mathrm{CHCH}_{2} \underline{\mathrm{H}}_{2} \mathrm{CO}\right), 2.18 \quad\left(1 \mathrm{H}, \quad \mathrm{CHC}_{2} \mathrm{CH}_{2} \mathrm{CO}\right), \quad 1.95 \quad\left(1 \mathrm{H}, \quad \mathrm{CHC}_{2} \mathrm{CH}_{2} \mathrm{CO}\right), \quad 1.60 \quad(2 \mathrm{H}$, $\left.\mathrm{OCH}_{2} \mathrm{CH}_{2} \mathrm{CH}_{2} \mathrm{CH}_{2}\right), 1.35\left(18 \mathrm{H}, \mathrm{OCH}_{2} \mathrm{CH}_{2} \mathrm{CH}_{2} \mathrm{CH}_{3}\right), 0.92-0.81\left(12 \mathrm{H}, \mathrm{CH}_{2} \mathrm{CH}_{2} \mathrm{CH}_{3}\right.$ and $\left.\mathrm{Si}\left(\mathrm{CH}_{3}\right)_{2}\left(\mathrm{CH}_{3}\right)_{3}\right), 0.02$ $\left(3 \mathrm{H}, \mathrm{Si}\left(\mathrm{C}_{3}\right)_{2}\left(\mathrm{CH}_{3}\right)_{3}\right), 0.02\left(3 \mathrm{H}, \mathrm{Si}\left(\mathrm{C}_{3}\right)_{2}\left(\mathrm{CH}_{3}\right)_{3}\right) .{ }^{13} \mathrm{C} \mathrm{NMR}\left(500 \mathrm{MHz}, \mathrm{CDCl}_{3}, \delta\right): 173.20,135.66,128.62$, $71.05,66.70,64.44,30.72,30.16,29.58,25.75,19.20,18.30,13.76,-4.91,-5.37$.

\subsubsection{OGA}

Synthesis of $\mathrm{H}_{2} \underline{\mathrm{N}-\mathrm{OGA}-\mathrm{COOH}}$. L-glutamic acid $(25.0 \mathrm{~g}, 169.92 \mathrm{mmol})$ was suspended in a mixture of tertbutanol $(130 \mathrm{~mL})$ and 1-octanol $(100 \mathrm{~mL})$. After heated to $40{ }^{\circ} \mathrm{C}$, concentrated $\mathrm{H}_{2} \mathrm{SO}_{4}(95 \%, 10.0 \mathrm{~mL})$ was added dropwise. The temperature was raised to $65^{\circ} \mathrm{C}$ for $120 \mathrm{~min}$. Triethylamine $(10.0 \mathrm{~mL})$ was then added dropwise to the reaction mixture rapidly. The reaction was diluted with water $(35 \mathrm{~mL})$ and methanol $(65 \mathrm{ml})$. Additional triethylamine $(40 \mathrm{~mL})$ was added, and the mixture was stirred for 30 minutes. The resultant precipitate was filtered out and resuspended in distilled water $\left(300 \mathrm{~mL}, 45^{\circ} \mathrm{C}\right)$. Finally, the obtained product was filtered out, washed by water and methanol, and dried at $25^{\circ} \mathrm{C}$ in vacuo $(30.8 \mathrm{~g}, 70 \%$ yield).

Synthesis of HO-OGA-COOH. $\mathrm{H}_{2} \mathrm{~N}-\mathrm{OGA}-\mathrm{COOH}(30.00 \mathrm{~g}, 115.67 \mathrm{mmol})$ was suspended in a mixture of 1,4dioxane, acetic acid, and deionized water $(300 \mathrm{~mL}$, volume ratio $3: 1: 1)$. The mixture was heated to $60{ }^{\circ} \mathrm{C}$, and tert-butyl nitrite (30.0 mL, $250.19 \mathrm{mmol})$ was added dropwise under stirring for $120 \mathrm{~min}$. The reaction solution was diluted by deionized water $(300 \mathrm{~mL})$ and extracted by ethyl acetate $(200 \mathrm{~mL})$ three times. The organic layers were collected, combined, and extracted with deionized water. The solvent was removed, and the obtained product was used directly without further purification.

Synthesis of HO-OGA. HO-OGA-COOH (30.00 g, $115.23 \mathrm{mmol})$ was dissolved in DMF (300 mL) and treated 
with $\mathrm{K}_{2} \mathrm{CO}_{3}(7.95 \mathrm{~g}, 57.62 \mathrm{mmol})$ for $1 \mathrm{~h}$ at room temperature. Benzyl bromide $(23.64 \mathrm{~g}, 138.28 \mathrm{mmol})$ was added and stirred at room temperature overnight. The reaction was then diluted by deionized water $(300 \mathrm{~mL})$ and extracted with ethyl acetate $(\mathrm{EtOAc}, 3 \times 200 \mathrm{~mL})$. The combined organic layers were further washed with deionized water $(3 \times 100 \mathrm{~mL})$ and dried with $\mathrm{Na}_{2} \mathrm{SO}_{4}$. After removal of the solvent, the crude material was purified by automated column chromatography using hexane/EtOAc (gradient 100/0 to 90/10) as eluent. The pure product was obtained as a colorless oil (26.25 g, 65\% yield). ${ }^{1} \mathrm{H}-\mathrm{NMR}\left(500 \mathrm{MHz}, \mathrm{CDCl}_{3}, \delta\right): 7.40-7.30$ $(5 \mathrm{H}, \operatorname{Ar}-\underline{H}), 5.21\left(2 \mathrm{H}, \operatorname{Ar}-\underline{C H}_{2} \mathrm{O}\right), 4.23\left(1 \mathrm{H}, \mathrm{HOC} \underline{H}\left(\mathrm{CH}_{2}\right) \mathrm{CO}\right), 4.05\left(2 \mathrm{H}, \mathrm{OC}_{2} \mathrm{CH}_{2} \mathrm{CH}_{2}\right), 2.45(2 \mathrm{H}$, $\left.\mathrm{CHCH}_{2} \underline{\mathrm{H}}_{2} \mathrm{CO}\right), 2.18\left(1 \mathrm{H}, \mathrm{CHC}_{2} \mathrm{CH}_{2} \mathrm{CO}\right), 1.95\left(1 \mathrm{H}, \mathrm{CHC}_{2} \mathrm{CH}_{2} \mathrm{CO}\right), 1.60\left(2 \mathrm{H}, \mathrm{OCH}_{2} \mathrm{CH}_{2} \mathrm{CH}_{2} \mathrm{CH}_{2}\right), 1.39-$ $1.15\left(10 \mathrm{H}, \mathrm{OCH}_{2} \mathrm{CH}_{2}\left(\mathrm{C}_{2}\right)_{5} \mathrm{CH}_{3}\right), 0.88\left(\mathrm{CH}_{2} \mathrm{CH}_{2} \underline{\mathrm{C}}_{3}\right)$.

Synthesis of OGA. HO-OGA-Bn (12.00 g, $34.24 \mathrm{mmol})$ and imidazole (5.82 g, $85.59 \mathrm{mmol})$ were dissolved in dried DMF $(100 \mathrm{~mL})$ in a $250 \mathrm{~mL}$ round bottom flask under argon atmosphere. Tertbutyldimethylsilyl chloride (TBDMSCl, $10.32 \mathrm{~g}, 68.48 \mathrm{mmol}$ ) were then added and stirred at room temperature overnight. The mixture was quenched by saturated $\mathrm{NaHCO}_{3}(200 \mathrm{~mL})$ and extracted with EtOAc $(3 \times 200 \mathrm{~mL})$. The combined organic layers were dried over $\mathrm{Na}_{2} \mathrm{SO}_{4}$ and the solvent was removed. The crude material was further purified by automated column chromatography using hexane/EtOAc (gradient 100/0 to 95/5) as eluent. Pure product was obtained as a colorless oil (15.28 g, 96\% yield). ${ }^{1} \mathrm{H}-\mathrm{NMR}\left(500 \mathrm{MHz}, \mathrm{CDCl}_{3}, \delta\right): 7.40-7.30(5 \mathrm{H}, \mathrm{Ar}-\underline{H})$, $5.21\left(2 \mathrm{H}, \mathrm{Ar}-\mathrm{C} \underline{H}_{2} \mathrm{O}\right), 4.30\left(1 \mathrm{H}, \mathrm{HOC} \underline{H}\left(\mathrm{CH}_{2}\right) \mathrm{CO}\right), 4.03\left(2 \mathrm{H}, \mathrm{OC}_{2} \mathrm{CH}_{2} \mathrm{CH}_{2}\right), 2.45\left(2 \mathrm{H}, \mathrm{CHCH}_{2} \mathrm{C} \underline{H}_{2} \mathrm{CO}\right), 2.18$ $\left(1 \mathrm{H}, \quad \mathrm{CHC} \underline{H}_{2} \mathrm{CH}_{2} \mathrm{CO}\right), 1.95\left(1 \mathrm{H}, \quad \mathrm{CHCH}_{2} \mathrm{CH}_{2} \mathrm{CO}\right), \quad 1.60 \quad\left(2 \mathrm{H}, \mathrm{OCH}_{2} \underline{\mathrm{C}}_{2} \mathrm{CH}_{2} \mathrm{CH}_{2}\right), \quad 1.39-1.15 \quad(10 \mathrm{H}$, $\left.\mathrm{OCH}_{2} \mathrm{CH}_{2}\left(\mathrm{C}_{2}\right)_{5} \mathrm{CH}_{3}\right), 0.92-0.81\left(12 \mathrm{H}, \mathrm{CH}_{2} \mathrm{CH}_{2} \mathrm{C}_{3}\right.$ and $\left.\mathrm{Si}\left(\mathrm{CH}_{3}\right)_{2}\left(\mathrm{C}_{3}\right)_{3}\right), 0.02\left(3 \mathrm{H}, \mathrm{Si}\left(\mathrm{C}_{3}\right)_{2}\left(\mathrm{CH}_{3}\right)_{3}\right), 0.02$ $\left(3 \mathrm{H}, \mathrm{Si}\left(\mathrm{CH}_{3}\right)_{2}\left(\mathrm{CH}_{3}\right)_{3}\right) .{ }^{13} \mathrm{C} \mathrm{NMR}\left(500 \mathrm{MHz}, \mathrm{CDCl}_{3}, \delta\right):$ 173.20, 135.66, 128.62, 71.05, 66.70, 64.76, 31.85, $30.15,29.58,29.28,29.24,28.67,25.98,25.75,22.70,18.30,14.15,-4.91,-5.37$.

\subsubsection{DGA}

Synthesis of $\mathrm{H}_{2} \mathrm{~N}$-DGA-COOH. L-glutamic acid $(25.0 \mathrm{~g}, 169.92 \mathrm{mmol})$ was suspended in a mixture of tertbutanol $(130 \mathrm{~mL})$ and 1-dodecanol $(130 \mathrm{~mL})$. After heated to $40{ }^{\circ} \mathrm{C}$, concentrated $\mathrm{H}_{2} \mathrm{SO}_{4}(95 \%, 10.0 \mathrm{~mL})$ is added dropwise. The temperature was raised to $65^{\circ} \mathrm{C}$ for $120 \mathrm{~min}$. Triethylamine $(10.0 \mathrm{~mL})$ was then added dropwise to the reaction mixture rapidly. The reaction was diluted with water $(35 \mathrm{~mL})$ and methanol $(65 \mathrm{ml})$. Additional triethylamine $(40 \mathrm{~mL})$ was added, and the mixture was stirred for 30 minutes. The resultant precipitate was filtered out and resuspended in distilled water $\left(300 \mathrm{~mL}, 45^{\circ} \mathrm{C}\right)$. Finally, the obtained product was filtered out, washed by water and methanol, and dried at $25^{\circ} \mathrm{C}$ in vacuo $(38.8 \mathrm{~g}, 71 \%$ yield $)$.

Synthesis of HO-DGA-COOH. $\mathrm{H}_{2} \mathrm{~N}-\mathrm{DGA}-\mathrm{COOH}(30.00 \mathrm{~g}, 95.10 \mathrm{mmol})$ was suspended in a mixture of 1,4- 
dioxane, acetic acid, and deionized water $(300 \mathrm{~mL}$, volume ratio $3: 1: 1)$. The mixture was heated to $60{ }^{\circ} \mathrm{C}$, and tert-butyl nitrite $(30.0 \mathrm{~mL}, 250.19 \mathrm{mmol})$ was added dropwise under stirring for $120 \mathrm{~min}$. The reaction solution was diluted by deionized water $(300 \mathrm{~mL})$ and extracted with ethyl acetate $(200 \mathrm{~mL})$ three times. The organic layers were collected, combined, and extracted by deionized water. The solvent was removed, and the obtained product was used directly without further purification.

Synthesis of HO-DGA. HO-DGA-COOH (30.00 g, $94.80 \mathrm{mmol})$ was dissolved in DMF (300 mL) and treated with $\mathrm{K}_{2} \mathrm{CO}_{3}(6.54 \mathrm{~g}, 47.40 \mathrm{mmol})$ for $1 \mathrm{~h}$ at room temperature. Benzyl bromide (19.45 g, $\left.113.76 \mathrm{mmol}\right)$ was added and stirred at room temperature overnight. The reaction was then diluted by deionized water $(300 \mathrm{~mL})$ and extracted with ethyl acetate $($ EtOAc, $3 \times 200 \mathrm{~mL})$. The combined organic layers were further washed with deionized water $(3 \times 100 \mathrm{~mL})$ and dried with $\mathrm{Na}_{2} \mathrm{SO}_{4}$. After removal of the solvent, the crude material was purified by automated column chromatography using hexane/EtOAc (gradient 100/0 to 90/10) as eluent. The pure product was obtained as a colorless oil $\left(23.20 \mathrm{~g}, 60 \%\right.$ yield). ${ }^{1} \mathrm{H}-\mathrm{NMR}\left(500 \mathrm{MHz}, \mathrm{CDCl}_{3}, \delta\right): 7.40-7.30$ $(5 \mathrm{H}, \operatorname{Ar}-\underline{H}), 5.21\left(2 \mathrm{H}, \operatorname{Ar}-\underline{C H}_{2} \mathrm{O}\right), 4.23\left(1 \mathrm{H}, \mathrm{HOC} \underline{H}\left(\mathrm{CH}_{2}\right) \mathrm{CO}\right), 4.05\left(2 \mathrm{H}, \mathrm{OC}_{2} \mathrm{CH}_{2} \mathrm{CH}_{2}\right), 2.45(2 \mathrm{H}$, $\left.\mathrm{CHCH}_{2} \underline{\mathrm{H}}_{2} \mathrm{CO}\right), 2.18\left(1 \mathrm{H}, \mathrm{CHC} \underline{\mathrm{H}}_{2} \mathrm{CH}_{2} \mathrm{CO}\right), 1.95\left(1 \mathrm{H}, \mathrm{CHC}_{2} \mathrm{CH}_{2} \mathrm{CO}\right), 1.60\left(2 \mathrm{H}, \mathrm{OCH}_{2} \underline{\mathrm{CH}}_{2} \mathrm{CH}_{2} \mathrm{CH}_{2}\right), 1.39-$ $1.15\left(18 \mathrm{H}, \mathrm{OCH}_{2} \mathrm{CH}_{2}\left(\mathrm{C}_{2}\right)_{9} \mathrm{CH}_{3}\right), 0.88\left(\mathrm{CH}_{2} \mathrm{CH}_{2} \underline{\mathrm{C}}_{3}\right)$.

Synthesis of DGA. HO-DGA-Bn (15.00 g, $36.89 \mathrm{mmol})$ and imidazole $(6.27 \mathrm{~g}, 92.24 \mathrm{mmol})$ were dissolved in dried DMF $(100 \mathrm{~mL})$ in a $250 \mathrm{~mL}$ round bottom flask under argon atmosphere. Tertbutyldimethylsilyl chloride (TBDMSCl, $11.18 \mathrm{~g}, 73.78 \mathrm{mmol}$ ) were then added and stirred at room temperature overnight. The mixture was quenched by saturated $\mathrm{NaHCO}_{3}(200 \mathrm{~mL})$ and extracted with EtOAc $(3 \times 200 \mathrm{~mL})$. The combined organic layers were dried over $\mathrm{Na}_{2} \mathrm{SO}_{4}$ and the solvent was removed. The crude material was further purified by automated column chromatography using hexane/EtOAc (gradient 100/0 to 95/5) as eluent. Pure product was obtained as a colorless oil (17.30 g, 90\% yield). ${ }^{1} \mathrm{H}-\mathrm{NMR}\left(500 \mathrm{MHz}, \mathrm{CDCl}_{3}, \delta\right): 7.40-7.30(5 \mathrm{H}, \mathrm{Ar}-\underline{H})$, $5.21\left(2 \mathrm{H}, \mathrm{Ar}-\mathrm{C} \underline{H_{2}} \mathrm{O}\right), 4.30\left(1 \mathrm{H}, \mathrm{HOC} \underline{H}\left(\mathrm{CH}_{2}\right) \mathrm{CO}\right), 4.03\left(2 \mathrm{H}, \mathrm{OC}_{2} \mathrm{CH}_{2} \mathrm{CH}_{2}\right), 2.45\left(2 \mathrm{H}, \mathrm{CHCH}_{2} \underline{\mathrm{H}}_{2} \mathrm{CO}\right), 2.18$ $\left(1 \mathrm{H}, \mathrm{CHC} \mathrm{H}_{2} \mathrm{CH}_{2} \mathrm{CO}\right), 1.95\left(1 \mathrm{H}, \mathrm{CHCH}_{2} \mathrm{CH}_{2} \mathrm{CO}\right), 1.60 \quad\left(2 \mathrm{H}, \mathrm{OCH}_{2} \mathrm{CH}_{2} \mathrm{CH}_{2} \mathrm{CH}_{2}\right), \quad 1.39-1.15$ (18H, $\left.\mathrm{OCH}_{2} \mathrm{CH}_{2}\left(\mathrm{C}_{2}\right)_{9} \mathrm{CH}_{3}\right), 0.92-0.81\left(12 \mathrm{H}, \mathrm{CH}_{2} \mathrm{CH}_{2} \mathrm{C}_{3}\right.$ and $\left.\mathrm{Si}\left(\mathrm{CH}_{3}\right)_{2}\left(\mathrm{C}_{3}\right)_{3}\right), 0.02\left(3 \mathrm{H}, \mathrm{Si}\left(\mathrm{C}_{3}\right)_{2}\left(\mathrm{CH}_{3}\right)_{3}\right), 0.02$ $\left(3 \mathrm{H}, \mathrm{Si}\left(\mathrm{CH}_{3}\right)_{2}\left(\mathrm{CH}_{3}\right)_{3}\right) .{ }^{13} \mathrm{C} \mathrm{NMR}\left(500 \mathrm{MHz}, \mathrm{CDCl}_{3}, \delta\right):$ 173.20, 135.66, 128.62, 71.05, 66.70, 64.76, 31.90, $30.15,29.72$, 29.70, 29.65, 29.59, 29.56, 29.42, 29.34, 28.68, 25.98, 25.75, 22.70, 18.30, 14.15, -4.91, -5.37.

\subsubsection{HGA}

Synthesis of $\mathrm{H}_{2} \mathrm{~N}-\mathrm{HGA}-\mathrm{COOH}$. L-glutamic acid $(25.0 \mathrm{~g}, 169.92 \mathrm{mmol})$ was suspended in a mixture of tertbutanol $(130 \mathrm{~mL})$ and 1-hexadecanol $(150 \mathrm{~g})$. After heated to $40{ }^{\circ} \mathrm{C}$, concentrated $\mathrm{H}_{2} \mathrm{SO}_{4}(95 \%, 10.0 \mathrm{~mL})$ is 
added dropwise. The temperature was raised to $65^{\circ} \mathrm{C}$ for $120 \mathrm{~min}$. Triethylamine $(10 \mathrm{~mL})$ was added dropwise to the reaction mixture rapidly. The reaction was diluted with water $(35 \mathrm{~mL})$ and methanol $(65 \mathrm{ml})$. Additional triethylamine $(40 \mathrm{~mL})$ was added, and the mixture was stirred for 30 minutes. The resultant precipitate was filtered out and resuspended in distilled water $\left(300 \mathrm{~mL}, 45^{\circ} \mathrm{C}\right)$. Finally, the obtained product was filtered out, washed by water, methanol, and ethyl ether, and dried at $25^{\circ} \mathrm{C}$ in vacuo ( $44.19 \mathrm{~g}, 70 \%$ yield).

Synthesis of HO-HGA-COOH. $\mathrm{H}_{2} \mathrm{~N}-\mathrm{HGA}-\mathrm{COOH}(40.00 \mathrm{~g}, 107.36 \mathrm{mmol})$ was suspended in a mixture of 1,4dioxane, acetic acid, and deionized water $(300 \mathrm{~mL}$, volume ratio $3: 1: 1)$. The mixture was heated to $60{ }^{\circ} \mathrm{C}$, and tert-butyl nitrite $(30 \mathrm{~mL}, 250.19 \mathrm{mmol})$ was added dropwise under stirring for $120 \mathrm{~min}$. The reaction solution was diluted by deionized water $(300 \mathrm{~mL})$, and extracted by ethyl acetate $(200 \mathrm{~mL})$ three times. The organic layers were collected, combined, and extracted with deionized water. The solvent was removed, and the obtained product was used directly without further purification.

Synthesis of HO-HGA. HO-HGA-COOH (30.00 g, $80.52 \mathrm{mmol})$ was dissolved in DMF (300 mL) and treated with $\mathrm{Cs}_{2} \mathrm{CO}_{3}(13.12 \mathrm{~g}, 40.26 \mathrm{mmol})$ for $1 \mathrm{~h}$ at $40^{\circ} \mathrm{C}$. Benzyl bromide (16.52 g, $\left.96.63 \mathrm{mmol}\right)$ was added under stirring, and maintained overnight. The reaction was then diluted by deionized water $(300 \mathrm{~mL})$ and extracted with ethyl acetate $($ EtOAc, $3 \times 200 \mathrm{~mL})$. The combined organic layers were further washed with deionized water $(3 \times 100 \mathrm{~mL})$ and dried with $\mathrm{Na}_{2} \mathrm{SO}_{4}$. After removal of the solvent, the crude material was purified by automated column chromatography using hexane/EtOAc (gradient 100/0 to 90/10) as eluent. The pure product was obtained as a colorless oil (18.63 g, $50 \%$ yield). ${ }^{1} \mathrm{H}-\mathrm{NMR}\left(500 \mathrm{MHz}, \mathrm{CDCl}_{3}, \delta\right): 7.40-7.30(5 \mathrm{H}, \mathrm{Ar}-\underline{H})$, $5.21\left(2 \mathrm{H}, \mathrm{Ar}-\underline{\mathrm{CH}}_{2} \mathrm{O}\right), 4.23\left(1 \mathrm{H}, \mathrm{HOC} \underline{H}\left(\mathrm{CH}_{2}\right) \mathrm{CO}\right), 4.05\left(2 \mathrm{H}, \mathrm{OC}_{2} \mathrm{CH}_{2} \mathrm{CH}_{2}\right), 2.45\left(2 \mathrm{H}, \mathrm{CHCH}_{2} \mathrm{CH} \underline{H}_{2} \mathrm{CO}\right), 2.18$ $\left(1 \mathrm{H}, \mathrm{CHC} \mathrm{H}_{2} \mathrm{CH}_{2} \mathrm{CO}\right), 1.95\left(1 \mathrm{H}, \mathrm{CHCH}_{2} \mathrm{CH}_{2} \mathrm{CO}\right), 1.60 \quad\left(2 \mathrm{H}, \mathrm{OCH}_{2} \mathrm{CH}_{2} \mathrm{CH}_{2} \mathrm{CH}_{2}\right), \quad 1.39-1.15$ (26H, $\left.\mathrm{OCH}_{2} \mathrm{CH}_{2}\left(\mathrm{C}_{2}\right)_{13} \mathrm{CH}_{3}\right), 0.88\left(\mathrm{CH}_{2} \mathrm{CH}_{2} \mathrm{C}_{3}\right)$.

Synthesis of HGA. HO-HGA-Bn (10.00 g, $21.61 \mathrm{mmol})$ and imidazole $(3.67 \mathrm{~g}, 54.03 \mathrm{mmol})$ were dissolved in dried DMF $(100 \mathrm{~mL})$ in a $250 \mathrm{~mL}$ round bottom flask under argon atmosphere. Tertbutyldimethylsilyl chloride (TBDMSCl, $6.51 \mathrm{~g}, 43.22 \mathrm{mmol}$ ) were then added and stirred at room temperature overnight. The mixture was quenched by saturated $\mathrm{NaHCO}_{3}(200 \mathrm{~mL})$ and extracted with EtOAc $(3 \times 200 \mathrm{~mL})$. The combined organic layers were dried over $\mathrm{Na}_{2} \mathrm{SO}_{4}$ and the solvent was removed. The crude material was further purified by automated column chromatography using hexane/EtOAc (gradient 100/0 to 95/5) as eluent. Pure product was obtained as a colorless oil $\left(9.77 \mathrm{~g}, 81 \%\right.$ yield). ${ }^{1} \mathrm{H}-\mathrm{NMR}\left(500 \mathrm{MHz}, \mathrm{CDCl}_{3}, \delta\right): 7.40-7.30(5 \mathrm{H}, \mathrm{Ar}-\underline{H})$, $5.21\left(2 \mathrm{H}, \mathrm{Ar}-\mathrm{C} \underline{H}_{2} \mathrm{O}\right), 4.30\left(1 \mathrm{H}, \mathrm{HOC} \underline{H}\left(\mathrm{CH}_{2}\right) \mathrm{CO}\right), 4.03\left(2 \mathrm{H}, \mathrm{OC}_{2} \mathrm{CH}_{2} \mathrm{CH}_{2}\right), 2.45\left(2 \mathrm{H}, \mathrm{CHCH}_{2} \underline{\mathrm{H}}_{2} \mathrm{CO}\right), 2.18$ $\left(1 \mathrm{H}, \quad \mathrm{CHC} \underline{H}_{2} \mathrm{CH}_{2} \mathrm{CO}\right), 1.95\left(1 \mathrm{H}, \mathrm{CHCH}_{2} \mathrm{CH}_{2} \mathrm{CO}\right), 1.60 \quad\left(2 \mathrm{H}, \quad \mathrm{OCH}_{2} \mathrm{CH}_{2} \mathrm{CH}_{2} \mathrm{CH}_{2}\right), \quad 1.39-1.15$ (26H, $\left.\mathrm{OCH}_{2} \mathrm{CH}_{2}\left(\mathrm{C}_{2}\right)_{13} \mathrm{CH}_{3}\right) ; 0.92-0.81\left(12 \mathrm{H}, \mathrm{CH}_{2} \mathrm{CH}_{2} \mathrm{C}_{3}\right.$ and $\left.\mathrm{Si}\left(\mathrm{CH}_{3}\right)_{2}\left(\mathrm{C}_{3}\right)_{3}\right), 0.02\left(3 \mathrm{H}, \mathrm{Si}\left(\mathrm{C}_{3}\right)_{2}\left(\mathrm{CH}_{3}\right)_{3}\right), 0.02$ 
(3H, $\left.\mathrm{Si}\left(\mathrm{CH}_{3}\right)_{2}\left(\mathrm{CH}_{3}\right)_{3}\right) .{ }^{13} \mathrm{C} \mathrm{NMR}\left(500 \mathrm{MHz}, \mathrm{CDCl}_{3}, \delta\right):$ 173.20, 135.66, 128.62, 71.05, 66.70, 64.76, 31.99, $30.15,29.78,29.73,29.66,29.59,29.56,29.44,29.34,28.68,25.98,25.75,22.70,18.30,14.15,-4.91,-5.37$.

\section{2 oCGA fragments}

\subsubsection{B 4}

Synthesis of BGA-COOH. BGA (12.00 g, $29.36 \mathrm{mmol})$ was dissolved in ethyl acetate $(120 \mathrm{~mL})$. Palladium (300 mg, 10\% on carbon) was then added. The mixture was stirred at room temperature under hydrogen atmosphere for $6 \mathrm{~h}$. The black suspension was filtered through a thick layer of celite. After removal of solvent, the product (BGA-COOH) was obtained in high purity (above 95\%) and used directly.

Synthesis of $\mathrm{B}_{2}$. HO-BGA (7.54 g, $\left.25.60 \mathrm{mmol}\right)$, BGA-COOH (8.97 g, $\left.28.16 \mathrm{mmol}\right)$, and DPTS (1.75 g) were mixed together into a round bottom flask. After transferring into a glove box, the mixture was dissolved in dry DCM. DIC $(10 \mathrm{~mL})$ was then added slowly at $0{ }^{\circ} \mathrm{C}$. The mixture was stirred at room temperature overnight. The precipitates were filtered out, and the solvent was removed. Purification by column chromatography using hexane/ethyl acetate $(95 / 5)$ as eluent gave the product $\mathrm{B}_{2}(15.10 \mathrm{~g}, 97 \%$ yield $) .{ }^{1} \mathrm{H}-\mathrm{NMR}\left(500 \mathrm{MHz}, \mathrm{CDCl}_{3}\right.$, $\delta): \quad 7.40-7.30 \quad(5 \mathrm{H}, \quad \operatorname{Ar}-\underline{H}), \quad 5.21-5.06 \quad\left(3 \mathrm{H}, \quad \operatorname{Ar}-\underline{C}_{2} \mathrm{O} \quad\right.$ and $\left.\mathrm{OC} \underline{H}\left(\mathrm{CH}_{2}\right) \mathrm{CO}\right), \quad 4.30 \quad(1 \mathrm{H}$, $\left.\left(\mathrm{CH}_{3}\right)_{3} \mathrm{Si}\left(\mathrm{CH}_{3}\right)_{2} \mathrm{OC} \underline{H}\left(\mathrm{CH}_{2}\right) \mathrm{CO}\right), 4.10-4.00\left(4 \mathrm{H}, \mathrm{OC}_{2} \mathrm{CH}_{2} \mathrm{CH}_{2}\right)$, 2.53-2.34 (4H, $\left.\mathrm{CHCH}_{2} \underline{\mathrm{H}}_{2} \mathrm{CO}\right), 2.32-2.08$ (4H, $\left.\mathrm{CHC}_{2} \mathrm{CH}_{2} \mathrm{CO}\right), 1.60\left(4 \mathrm{H}, \mathrm{OCH}_{2} \underline{\mathrm{H}}_{2} \mathrm{CH}_{2} \mathrm{CH}_{2}\right), 1.39-1.15\left(4 \mathrm{H}, \mathrm{OCH}_{2} \mathrm{CH}_{2} \underline{\mathrm{CH}}_{2} \mathrm{CH}_{3}\right), 0.94-0.82$ (15H, $\mathrm{CH}_{2} \mathrm{CH}_{2} \mathrm{C}_{3}$ and $\left.\mathrm{Si}\left(\mathrm{CH}_{3}\right)_{2}\left(\mathrm{C}_{3}\right)_{3}\right), 0.02\left(6 \mathrm{H}, \mathrm{Si}\left(\mathrm{C}_{3}\right)_{2}\left(\mathrm{CH}_{3}\right)_{3}\right)$.

Synthesis of $\mathrm{B}_{2}=\mathrm{COOH} . \mathrm{B}_{2}(3.27 \mathrm{~g}, 5.50 \mathrm{mmol})$ was dissolved in ethyl acetate. Palladium $(53 \mathrm{mg}, 10 \%$ on carbon) was then added. The mixture was stirred at room temperature under hydrogen atmosphere for $6 \mathrm{~h}$. The black suspension was filtered through a thick layer of celite. After removal of solvent in vacuo, product $\left(\mathrm{B}_{2^{-}}\right.$ $\mathrm{COOH}$ ) was obtained in high purity (above 95\%) and used directly.

Synthesis of HO-B${ }_{2} . \mathrm{B}_{2}(4.00 \mathrm{~g}, 6.72 \mathrm{mmol})$ was dissolved in anhydrous $\mathrm{CH}_{2} \mathrm{Cl}_{2} . \mathrm{BF}_{3}$-etherate $(5.0 \mathrm{~mL})$ was then slowly added at $0{ }^{\circ} \mathrm{C}$. The mixture was allowed to return to room temperature and was further stirred for $4 \mathrm{~h}$. The reaction was quenched by adding saturated $\mathrm{NaHCO}_{3}(100 \mathrm{~mL})$. The organic layer was separated and dried with $\mathrm{Na}_{2} \mathrm{SO}_{4}$. After removal of solvent, the crude product was purified by column chromatography using $n$-hexane/ethyl acetate (85/15) as eluent to give the pure material $\mathrm{HO}-\mathrm{B}_{2}\left(2.94 \mathrm{~g}, 91 \%\right.$ yield). ${ }^{1} \mathrm{H}-\mathrm{NMR}(500$ $\left.\mathrm{MHz}, \mathrm{CDCl}_{3}, \delta\right):$ 7.40-7.30 (5H, Ar- $\left.\underline{H}\right), 5.21-5.06\left(3 \mathrm{H}, \mathrm{Ar}-\underline{\mathrm{H}}_{2} \mathrm{O}, \mathrm{OC} \underline{H}\left(\mathrm{CH}_{2}\right) \mathrm{CO}\right), 4.30\left(1 \mathrm{H}, \mathrm{HOC} \underline{H}\left(\mathrm{CH}_{2}\right) \mathrm{CO}\right)$, 4.10-4.00 (4H, OC $\underline{H}_{2} \mathrm{CH}_{2} \mathrm{CH}_{2}$ ), 2.53-2.34 (4H, $\left.\mathrm{CHCH}_{2} \underline{\mathrm{C}}_{2} \mathrm{CO}\right), 2.32-2.08$ (4H, $\left.\mathrm{CHCH}_{2} \mathrm{CH}_{2} \mathrm{CO}\right), 1.60$ (4H, $\left.\mathrm{OCH}_{2} \underline{\mathrm{C}}_{2} \mathrm{CH}_{2} \mathrm{CH}_{2}\right), 1.39-1.15\left(4 \mathrm{H}, \mathrm{OCH}_{2} \mathrm{CH}_{2} \mathrm{C}_{2} \mathrm{CH}_{3}\right), 0.92-0.81\left(6 \mathrm{H}, \mathrm{CH}_{2} \mathrm{CH}_{2} \mathrm{C}_{3}\right)$. 
Synthesis of $\mathrm{B}_{4 .}$ HO-B $2(2.40 \mathrm{~g}, 5.01 \mathrm{mmol}), \mathrm{B}_{2}-\mathrm{COOH}(3.27 \mathrm{~g}, 5.50 \mathrm{mmol})$, and DPTS $(0.34 \mathrm{~g})$ were mixed together into a round bottom flask. After transferring into a glove box, the mixture was dissolved in dry DCM. DIC $(2.0 \mathrm{~mL})$ was then added slowly at $0{ }^{\circ} \mathrm{C}$. The mixture was stirred at room temperature overnight. The reaction was filtered, and the solvent was removed. Purification by column chromatography using hexane/ethyl acetate (90/10) as eluent gave the product $\mathrm{B}_{4}\left(4.51 \mathrm{~g}, 94 \%\right.$ yield). ${ }^{1} \mathrm{H}-\mathrm{NMR}\left(500 \mathrm{MHz}, \mathrm{CDCl}_{3}\right.$, $\delta): \quad 7.40-7.30 \quad(5 \mathrm{H}, \quad \operatorname{Ar}-\underline{H}), \quad 5.21-5.06 \quad\left(5 \mathrm{H}, \quad \operatorname{Ar}-\underline{C}_{2} \mathrm{O} \quad\right.$ and $\left.\quad \mathrm{OC} \underline{H}\left(\mathrm{CH}_{2}\right) \mathrm{CO}\right), \quad 4.30 \quad(1 \mathrm{H}$, $\left.\left(\mathrm{CH}_{3}\right)_{3} \mathrm{Si}\left(\mathrm{CH}_{3}\right)_{2} \mathrm{OC} \underline{H}\left(\mathrm{CH}_{2}\right) \mathrm{CO}\right), 4.10-4.00\left(8 \mathrm{H}, \mathrm{OCH}_{2} \mathrm{CH}_{2} \mathrm{CH}_{2}\right), 2.53-2.34\left(8 \mathrm{H}, \mathrm{CHCH}_{2} \mathrm{CH}_{2} \mathrm{CO}\right), 2.32-2.08$ $\left(8 \mathrm{H}, \mathrm{CHC} \underline{H}_{2} \mathrm{CH}_{2} \mathrm{CO}\right), 1.60\left(8 \mathrm{H}, \mathrm{OCH}_{2} \underline{\mathrm{H}}_{2} \mathrm{CH}_{2} \mathrm{CH}_{2}\right), 1.39-1.15\left(8 \mathrm{H}, \mathrm{OCH}_{2} \mathrm{CH}_{2} \mathrm{C}_{2} \mathrm{CH}_{3}\right) ; 0.94-0.82(21 \mathrm{H}$, $\mathrm{CH}_{2} \mathrm{CH}_{2} \mathrm{C}_{3}$ and $\left.\mathrm{Si}\left(\mathrm{CH}_{3}\right)_{2}\left(\mathrm{C}_{3}\right)_{3}\right), 0.02\left(6 \mathrm{H}, \mathrm{Si}\left(\mathrm{C}_{3}\right)_{2}\left(\mathrm{CH}_{3}\right)_{3}\right) . \mathrm{MS}$ (MALDI-ToF): [M+Na] $]^{+} \mathrm{Cal}$ 989.49; Obs 989.42.

\subsection{2 $\mathrm{O}_{4}$}

Synthesis of OGA-COOH. OGA (15.72 g, $33.83 \mathrm{mmol})$ was dissolved in ethyl acetate $(150 \mathrm{~mL})$. Palladium (300 mg, 10\% on carbon) was then added. The mixture was stirred at room temperature under hydrogen atmosphere for $6 \mathrm{~h}$. The black suspension was filtered through a thick layer of celite. After removal of solvent, the product (OGA-COOH) was obtained in high purity (above 95\%) and used directly.

Synthesis of $\mathrm{O}_{2}=$ HO-OGA (11.29 g, $\left.30.21 \mathrm{mmol}\right)$, OGA-COOH (12.36 g, $\left.33.00 \mathrm{mmol}\right)$, and DPTS (2.16 g) were mixed together into a round bottom flask. After transferring into a glove box, the mixture was dissolved in dry DCM. DIC $(10 \mathrm{~mL})$ was then added slowly at $0{ }^{\circ} \mathrm{C}$. The mixture was stirred at room temperature overnight. The precipitates were filtered out, and the solvent was removed. Purification by column chromatography using hexane/ethyl acetate (95/5) as eluent gave the product $\mathrm{O}_{2}\left(18.40 \mathrm{~g}, 81 \%\right.$ yield). ${ }^{1} \mathrm{H}$ NMR (500 MHz, $\left.\mathrm{CDCl}_{3}, \delta\right): 7.40-7.30(5 \mathrm{H}, \mathrm{Ar}-\underline{H}), 5.21-5.06\left(3 \mathrm{H}, \mathrm{Ar}-\underline{\mathrm{C}}_{2} \mathrm{O}\right.$ and $\left.\mathrm{OC} \underline{\mathrm{H}}\left(\mathrm{CH}_{2}\right) \mathrm{CO}\right), 4.30(1 \mathrm{H}$, $\left.\left(\mathrm{CH}_{3}\right)_{3} \mathrm{Si}\left(\mathrm{CH}_{3}\right)_{2} \mathrm{OC} \underline{H}\left(\mathrm{CH}_{2}\right) \mathrm{CO}\right), 4.10-4.00\left(4 \mathrm{H}, \mathrm{OC}_{2} \mathrm{CH}_{2} \mathrm{CH}_{2}\right), 2.53-2.34\left(4 \mathrm{H}, \mathrm{CHCH}_{2} \mathrm{CH}_{2} \mathrm{CO}\right), 2.32-2.08$ (4H, $\left.\mathrm{CHC} \underline{H}_{2} \mathrm{CH}_{2} \mathrm{CO}\right), 1.60\left(4 \mathrm{H}, \mathrm{OCH}_{2} \underline{\mathrm{H}}_{2} \mathrm{CH}_{2} \mathrm{CH}_{2}\right), 1.39-1.15\left(20 \mathrm{H}, \mathrm{OCH}_{2} \mathrm{CH}_{2}\left(\mathrm{C}_{2}\right)_{5} \mathrm{CH}_{3}\right), 0.94-0.82(15 \mathrm{H}$, $\mathrm{CH}_{2} \mathrm{CH}_{2} \underline{\mathrm{C}}_{3}$ and $\left.\mathrm{Si}\left(\mathrm{CH}_{3}\right)_{2}\left(\mathrm{C}_{3}\right)_{3}\right), 0.02\left(6 \mathrm{H}, \mathrm{Si}\left(\mathrm{C}_{\mathrm{H}_{3}}\right)_{2}\left(\mathrm{CH}_{3}\right)_{3}\right)$.

Synthesis of $\mathrm{O}_{2}$-COOH. $\mathrm{O}_{2}(7.75 \mathrm{~g}, 10.97 \mathrm{mmol})$ was dissolved in ethyl acetate $(70 \mathrm{~mL})$. Palladium $(200 \mathrm{mg}$, $10 \%$ on carbon) was then added. The mixture was stirred at room temperature under hydrogen atmosphere for 6h. The black suspension was filtered through a thick layer of celite. After removal of solvent, the product $\left(\mathrm{O}_{2}-\mathrm{COOH}\right)$ was obtained in high purity (above $\left.95 \%\right)$ and used directly.

Synthesis of $\mathrm{HO}_{2} \mathrm{O}_{2} . \mathrm{O}_{2}(10.00 \mathrm{~g}, 14.14 \mathrm{mmol})$ was dissolved in anhydrous $\mathrm{CH}_{2} \mathrm{Cl}_{2} . \mathrm{BF}_{3}$-etherate $(10.0 \mathrm{~mL})$ 
was then slowly added at $0{ }^{\circ} \mathrm{C}$. The mixture was allowed to return to room temperature and was further stirred for $4 \mathrm{~h}$. The reaction was quenched by adding saturated $\mathrm{NaHCO}_{3}(150 \mathrm{~mL})$. The organic layer was separated and dried with $\mathrm{Na}_{2} \mathrm{SO}_{4}$. After removal of solvent, the crude product was purified by column chromatography using $n$-hexane/ethyl acetate (85/15) as eluent to give the pure material $\mathrm{HO}-\mathrm{O}_{2}\left(7.80 \mathrm{~g}, 93 \%\right.$ yield). ${ }^{1} \mathrm{H}-\mathrm{NMR}$ $\left(500 \mathrm{MHz}, \mathrm{CDCl}_{3}, \delta\right): 7.40-7.30(5 \mathrm{H}, \mathrm{Ar}-\underline{H}), 5.21-5.06\left(3 \mathrm{H}, \operatorname{Ar}-\underline{C}_{2} \mathrm{O}, \mathrm{OC} \underline{H}\left(\mathrm{CH}_{2}\right) \mathrm{CO}\right), 4.30(1 \mathrm{H}$, $\left.\mathrm{HOC} \underline{H}\left(\mathrm{CH}_{2}\right) \mathrm{CO}\right), \quad 4.10-4.00 \quad\left(4 \mathrm{H}, \quad \mathrm{OC}_{\underline{H}} \mathrm{CH}_{2} \mathrm{CH}_{2}\right), 2.53-2.34 \quad\left(4 \mathrm{H}, \quad \mathrm{CHCH}_{2} \underline{\mathrm{C}}_{2} \mathrm{CO}\right), \quad 2.32-2.08 \quad(4 \mathrm{H}$, $\left.\mathrm{CHCH}_{2} \mathrm{CH}_{2} \mathrm{CO}\right), 1.60\left(4 \mathrm{H}, \mathrm{OCH}_{2} \mathrm{CH}_{2} \mathrm{CH}_{2} \mathrm{CH}_{2}\right), 1.39-1.15\left(20 \mathrm{H}, \mathrm{OCH}_{2} \mathrm{CH}_{2}\left(\mathrm{CH}_{2}\right)_{5} \mathrm{CH}_{3}\right), 0.92-0.81(6 \mathrm{H}$, $\left.\mathrm{CH}_{2} \mathrm{CH}_{2} \underline{\mathrm{C}}_{3}\right)$.

Synthesis of $\mathrm{O}_{4} . \mathrm{HO}-\mathrm{O}_{2}(5.91 \mathrm{~g}, 10.45 \mathrm{mmol}), \mathrm{O}_{2}-\mathrm{COOH}(6.77 \mathrm{~g}, 10.97 \mathrm{mmol})$, and DPTS (0.70 g) were mixed together into a round bottom flask. After transferring into a glove box, the mixture was dissolved in dry DCM. DIC $(5.0 \mathrm{~mL})$ was then added slowly at $0{ }^{\circ} \mathrm{C}$. The mixture was stirred at room temperature overnight. The precipitates were filtered out, and the solvent was removed. Purification by column chromatography using hexane/ethyl acetate (10/90) as eluent gave the product $\mathrm{O}_{4}\left(11.20 \mathrm{~g}, 92 \%\right.$ yield). ${ }^{1} \mathrm{H}-\mathrm{NMR}\left(500 \mathrm{MHz}, \mathrm{CDCl}_{3}\right.$,

$\delta): \quad 7.40-7.30 \quad(5 \mathrm{H}, \quad \operatorname{Ar}-\underline{H}), \quad 5.21-5.06 \quad\left(5 \mathrm{H}, \quad \operatorname{Ar}-\underline{C}_{2} \mathrm{O} \quad\right.$ and $\left.\quad \mathrm{OC} \underline{H}\left(\mathrm{CH}_{2}\right) \mathrm{CO}\right), \quad 4.30 \quad(1 \mathrm{H}$, $\left.\left(\mathrm{CH}_{3}\right)_{3} \mathrm{Si}\left(\mathrm{CH}_{3}\right)_{2} \mathrm{OC} \underline{H}\left(\mathrm{CH}_{2}\right) \mathrm{CO}\right), 4.10-4.00\left(8 \mathrm{H}, \mathrm{OC}_{2} \mathrm{CH}_{2} \mathrm{CH}_{2}\right), 2.53-2.34\left(8 \mathrm{H}, \mathrm{CHCH}_{2} \underline{\mathrm{H}}_{2} \mathrm{CO}\right), 2.32-2.08$ $\left(8 \mathrm{H}, \mathrm{CHC} \underline{H}_{2} \mathrm{CH}_{2} \mathrm{CO}\right), 1.60\left(8 \mathrm{H}, \mathrm{OCH}_{2} \underline{\mathrm{H}}_{2} \mathrm{CH}_{2} \mathrm{CH}_{2}\right), 1.39-1.15\left(40 \mathrm{H}, \mathrm{OCH}_{2} \mathrm{CH}_{2}\left(\mathrm{C}_{2}\right)_{5} \mathrm{CH}_{3}\right) ; 0.94-0.82(21 \mathrm{H}$, $\mathrm{CH}_{2} \mathrm{CH}_{2} \mathrm{C}_{3}$ and $\left.\mathrm{Si}\left(\mathrm{CH}_{3}\right)_{2}\left(\mathrm{C}_{3}\right)_{3}\right), 0.02\left(6 \mathrm{H}, \mathrm{Si}\left(\mathrm{C}_{\mathrm{H}_{3}}\right)_{2}\left(\mathrm{CH}_{3}\right)_{3}\right)$. MS (MALDI-ToF): [M+Na] $]^{+} \mathrm{Cal} 1213.74 ; \mathrm{Obs}$ 1213.92 .

\subsection{3 $\mathrm{D}_{4}$ and $\mathrm{D}_{6}$}

Synthesis of DGA-COOH. DGA $(22.3 \mathrm{~g}, 42.82 \mathrm{mmol})$ was dissolved in ethyl acetate $(250 \mathrm{~mL})$. Palladium (500 mg, 10\% on carbon) was then added. The mixture was stirred at room temperature under hydrogen atmosphere for $6 \mathrm{~h}$. The black suspension was filtered through a thick layer of celite. After removal of solvent, the product (DGA-COOH) was obtained in high purity (above 95\%) and used directly.

Synthesis of $\mathrm{D}_{2}$. HO-DGA (16.6 g, $\left.40.78 \mathrm{mmol}\right)$, DGA-COOH (18.35 g, $\left.42.60 \mathrm{mmol}\right)$, and DPTS (2.16 g) were mixed together into a round bottom flask. After transferring into a glove box, the mixture was dissolved in dry DCM. DIC $(16 \mathrm{~mL})$ was then added slowly at $0{ }^{\circ} \mathrm{C}$. The mixture was stirred at room temperature overnight. The precipitates were filtered out, and the solvent was removed. Purification by column chromatography using hexane/ethyl acetate (95/5) as eluent gave the product $\mathrm{D}_{2}\left(29.85 \mathrm{~g}, 89 \%\right.$, yield). ${ }^{1} \mathrm{H}$ NMR (500 MHz, $\left.\mathrm{CDCl}_{3}, \delta\right)$ : 7.40-7.30 (5H, Ar- $\left.\underline{H}\right), 5.21-5.06\left(3 \mathrm{H}, \mathrm{Ar}-\mathrm{C}_{2} \mathrm{O}\right.$ and $\left.\mathrm{OC} \underline{H}\left(\mathrm{CH}_{2}\right) \mathrm{CO}\right), 4.30(1 \mathrm{H}$, $\left.\left(\mathrm{CH}_{3}\right)_{3} \mathrm{Si}\left(\mathrm{CH}_{3}\right)_{2} \mathrm{OC} \underline{H}\left(\mathrm{CH}_{2}\right) \mathrm{CO}\right), 4.10-4.00\left(4 \mathrm{H}, \mathrm{OCH}_{2} \mathrm{CH}_{2} \mathrm{CH}_{2}\right)$, 2.53-2.34 (4H, $\left.\mathrm{CHCH}_{2} \mathrm{CH}_{2} \mathrm{CO}\right), 2.32-2.08$ 
(4H, $\left.\mathrm{CHC} \underline{H}_{2} \mathrm{CH}_{2} \mathrm{CO}\right), 1.60\left(4 \mathrm{H}, \mathrm{OCH}_{2} \underline{\mathrm{H}}_{2} \mathrm{CH}_{2} \mathrm{CH}_{2}\right), 1.39-1.15\left(36 \mathrm{H}, \mathrm{OCH}_{2} \mathrm{CH}_{2}\left(\mathrm{C}_{2}\right)_{9} \mathrm{CH}_{3}\right), 0.94-0.82(15 \mathrm{H}$, $\mathrm{CH}_{2} \mathrm{CH}_{2} \underline{\mathrm{C}}_{3}$ and $\left.\mathrm{Si}\left(\mathrm{CH}_{3}\right)_{2}\left(\mathrm{C}_{3}\right)_{3}\right), 0.02\left(6 \mathrm{H}, \mathrm{Si}\left(\mathrm{C}_{H_{3}}\right)_{2}\left(\mathrm{CH}_{3}\right)_{3}\right)$.

Synthesis of $\mathrm{D}_{2}-\mathrm{COOH} . \mathrm{D}_{2}(10.00 \mathrm{~g}, 12.21 \mathrm{mmol})$ was dissolved in ethyl acetate $(100 \mathrm{~mL})$. Palladium (129 $\mathrm{mg}, 10 \%$ on carbon) was then added. The mixture was stirred at room temperature under hydrogen atmosphere for $6 \mathrm{~h}$. The black suspension was filtered through a thick layer of celite. After removal of solvent, the product $\left(\mathrm{D}_{2}-\mathrm{COOH}\right)$ was obtained in high purity (above $95 \%$ ) and used directly.

Synthesis of $\mathrm{HO}-\mathrm{D}_{2} . \mathrm{D}_{2}(13.0 \mathrm{~g}, 15.87 \mathrm{mmol})$ was dissolved in anhydrous $\mathrm{CH}_{2} \mathrm{Cl}_{2} . \mathrm{BF}_{3}$-etherate $(10.0 \mathrm{~mL})$ was then slowly added at $0{ }^{\circ} \mathrm{C}$. The mixture was allowed to return to room temperature and was further stirred for 4h. The reaction was quenched by adding saturated $\mathrm{NaHCO}_{3}(200 \mathrm{~mL})$. The organic layer was separated and dried with $\mathrm{Na}_{2} \mathrm{SO}_{4}$. After removal of solvent, the crude product was purified by column chromatography using n-hexane/ethyl acetate (85/15) as eluent to give the pure material HO-D $\left(10.30 \mathrm{~g}, 92 \%\right.$ yield). ${ }^{1} \mathrm{H}-\mathrm{NMR}$ (500 $\left.\mathrm{MHz}, \mathrm{CDCl}_{3}, \delta\right):$ 7.40-7.30 (5H, Ar- $\left.\underline{H}\right), 5.21-5.06\left(3 \mathrm{H}, \mathrm{Ar}-\underline{\mathrm{C}}_{2} \mathrm{O}, \mathrm{OC} \underline{\mathrm{H}}\left(\mathrm{CH}_{2}\right) \mathrm{CO}\right), 4.30\left(1 \mathrm{H}, \mathrm{HOC} \underline{H}\left(\mathrm{CH}_{2}\right) \mathrm{CO}\right)$, 4.10-4.00 (4H, OC $\left.\underline{H}_{2} \mathrm{CH}_{2} \mathrm{CH}_{2}\right), 2.53-2.34$ (4H, $\left.\mathrm{CHCH}_{2} \underline{\mathrm{C}}_{2} \mathrm{CO}\right), 2.32-2.08\left(4 \mathrm{H}, \mathrm{CHC}_{2} \mathrm{CH}_{2} \mathrm{CO}\right), 1.60$ (4H, $\left.\mathrm{OCH}_{2} \mathrm{CH}_{2} \mathrm{CH}_{2} \mathrm{CH}_{2}\right), 1.39-1.15\left(36 \mathrm{H}, \mathrm{OCH}_{2} \mathrm{CH}_{2}\left(\mathrm{CH}_{2}\right)_{9} \mathrm{CH}_{3}\right), 0.92-0.81\left(6 \mathrm{H}, \mathrm{CH}_{2} \mathrm{CH}_{2} \mathrm{CH}_{3}\right)$.

Synthesis of $\mathrm{D}_{4} . \mathrm{HO}-\mathrm{D}_{2}(7.83 \mathrm{~g}, 12.21 \mathrm{mmol}), \mathrm{D}_{2}-\mathrm{COOH}(8.90 \mathrm{~g}, 12.21 \mathrm{mmol})$, and DPTS $(0.75 \mathrm{~g})$ were mixed together into a round bottom flask. After transferring into a glove box, the mixture was dissolved in dry DCM. DIC $(5 \mathrm{~mL})$ was then added slowly at $0{ }^{\circ} \mathrm{C}$. The mixture was stirred at room temperature overnight. The precipitates were filtered out, and the solvent was removed. Purification by column chromatography using hexane/ethyl acetate (10/90) as eluent gave the product $\mathrm{D}_{4}\left(15.10 \mathrm{~g}, 95 \%\right.$ yield). ${ }^{1} \mathrm{H}-\mathrm{NMR}\left(500 \mathrm{MHz}, \mathrm{CDCl}_{3}\right.$, $\delta): \quad 7.40-7.30 \quad(5 \mathrm{H}, \quad \mathrm{Ar}-\underline{H}), \quad 5.21-5.06 \quad\left(5 \mathrm{H}, \quad \mathrm{Ar}-\mathrm{C}_{2} \mathrm{O} \quad\right.$ and $\left.\quad \mathrm{OC} \underline{H}\left(\mathrm{CH}_{2}\right) \mathrm{CO}\right), \quad 4.30 \quad(1 \mathrm{H}$, $\left.\left(\mathrm{CH}_{3}\right)_{3} \mathrm{Si}\left(\mathrm{CH}_{3}\right)_{2} \mathrm{OC} \underline{H}\left(\mathrm{CH}_{2}\right) \mathrm{CO}\right), 4.10-4.00\left(8 \mathrm{H}, \mathrm{OC}_{2} \mathrm{CH}_{2} \mathrm{CH}_{2}\right)$, 2.53-2.34 (8H, $\left.\mathrm{CHCH}_{2} \mathrm{CH}_{2} \mathrm{CO}\right), 2.32-2.08$ (8H, $\left.\mathrm{CHC}_{2} \mathrm{CH}_{2} \mathrm{CO}\right), 1.60\left(8 \mathrm{H}, \mathrm{OCH}_{2} \underline{\mathrm{H}}_{2} \mathrm{CH}_{2} \mathrm{CH}_{2}\right), 1.39-1.15\left(72 \mathrm{H}, \mathrm{OCH}_{2} \mathrm{CH}_{2}\left(\mathrm{C}_{2}\right)_{9} \mathrm{CH}_{3}\right) ; 0.94-0.82(21 \mathrm{H}$, $\mathrm{CH}_{2} \mathrm{CH}_{2} \mathrm{C}_{3}$ and $\left.\mathrm{Si}\left(\mathrm{CH}_{3}\right)_{2}\left(\mathrm{C}_{3}\right)_{3}\right) ; 0.02\left(6 \mathrm{H}, \mathrm{Si}\left(\mathrm{C}_{\underline{H}_{3}}\right)_{2}\left(\mathrm{CH}_{3}\right)_{3}\right)$. MS (MALDI-ToF): [M+Na] ${ }^{+} \mathrm{Cal} 1437.98 ; \mathrm{Obs}$ 1437.88 .

Synthesis of $\mathrm{D}_{4}-\mathrm{COOH} . \mathrm{D}_{4}(5.07 \mathrm{~g}, 3.58 \mathrm{mmol})$ was dissolved in ethyl acetate $(50 \mathrm{~mL})$. Palladium $(200 \mathrm{mg}$, $10 \%$ on carbon) was then added. The mixture was stirred at room temperature under hydrogen atmosphere for 6h. The black suspension was filtered through a thick layer of celite. After removal of solvent, the product $\left(\mathrm{D}_{4}-\mathrm{COOH}\right)$ was obtained in high purity (above $95 \%$ ) and used directly.

Synthesis of $\mathrm{D}_{6} . \mathrm{HO}_{2} \mathrm{D}_{2}(1.56 \mathrm{~g}, 2.21 \mathrm{mmol}), \mathrm{D}_{4}-\mathrm{COOH}(3.22 \mathrm{~g}, 2.43 \mathrm{mmol})$, and DPTS $(0.75 \mathrm{~g})$ were mixed 
together into a round bottom flask. After transferring into a glove box, the mixture was dissolved in dry DCM. DIC ( $2 \mathrm{~mL})$ was then added slowly at $0{ }^{\circ} \mathrm{C}$. The mixture was stirred at room temperature overnight. The precipitates were filtered out, and the solvent was removed. Purification by column chromatography using hexane/ethyl acetate (85/15) as eluent gave the product $\mathrm{D}_{6}\left(4.23 \mathrm{~g}, 95 \%\right.$ yield). ${ }^{1} \mathrm{H}-\mathrm{NMR}\left(500 \mathrm{MHz}, \mathrm{CDCl}_{3}\right.$,

$\delta): \quad 7.40-7.30 \quad(5 \mathrm{H}, \quad \operatorname{Ar}-\underline{H}), \quad 5.21-5.06 \quad\left(7 \mathrm{H}, \quad \operatorname{Ar}-\underline{C}_{2} \mathrm{O} \quad\right.$ and $\left.\quad \mathrm{OC} \underline{H}\left(\mathrm{CH}_{2}\right) \mathrm{CO}\right), \quad 4.30 \quad(1 \mathrm{H}$, $\left.\left(\mathrm{CH}_{3}\right)_{3} \mathrm{Si}\left(\mathrm{CH}_{3}\right)_{2} \mathrm{OC} \underline{H}\left(\mathrm{CH}_{2}\right) \mathrm{CO}\right), 4.10-4.00\left(12 \mathrm{H}, \mathrm{OC}_{2} \mathrm{CH}_{2} \mathrm{CH}_{2}\right)$, 2.53-2.34 (12H, $\left.\mathrm{CHCH}_{2} \underline{\mathrm{H}}_{2} \mathrm{CO}\right), 2.32-2.08$ $\left(12 \mathrm{H}, \mathrm{CHCH}_{2} \mathrm{CH}_{2} \mathrm{CO}\right), 1.60\left(12 \mathrm{H}, \mathrm{OCH}_{2} \mathrm{CH}_{2} \mathrm{CH}_{2} \mathrm{CH}_{2}\right), 1.39-1.15\left(108 \mathrm{H}, \mathrm{OCH}_{2} \mathrm{CH}_{2}\left(\mathrm{CH}_{2}\right)_{9} \mathrm{CH}_{3}\right), 0.94-0.82$ $\left(27 \mathrm{H}, \mathrm{CH}_{2} \mathrm{CH}_{2} \underline{\mathrm{C}}_{3}\right.$ and $\left.\mathrm{Si}\left(\mathrm{CH}_{3}\right)_{2}\left(\mathrm{C}_{3}\right)_{3}\right), 0.02\left(6 \mathrm{H}, \mathrm{Si}\left(\mathrm{C}_{H_{3}}\right)_{2}\left(\mathrm{CH}_{3}\right)_{3}\right) . \mathrm{MS}$ (MALDI-ToF): [M+Na] $]^{+} \mathrm{Cal}$ 2034.40; Obs 2034.29.

\subsection{4 $\mathrm{H}_{4}$}

Synthesis of HGA-COOH. HGA (6.82 g, $11.83 \mathrm{mmol})$ was dissolved in ethyl acetate (70 mL). Palladium (300 $\mathrm{mg}, 10 \%$ on carbon) was then added. The mixture was stirred at room temperature under hydrogen atmosphere for $6 \mathrm{~h}$. The black suspension was filtered through a thick layer of celite. After removal of solvent, the product (HGA-COOH) was obtained in high purity (above 95\%) and used directly.

Synthesis of $\mathrm{H}_{2}$. HO-HGA (4.56 g, $\left.9.86 \mathrm{mmol}\right)$, HGA-COOH (5.76 g, $\left.11.80 \mathrm{mmol}\right)$, and DPTS (0.66 g) were mixed together into a round bottom flask. After transferring into a glove box, the mixture was dissolved in dry DCM. DIC $(4 \mathrm{~mL})$ was then added slowly at $0{ }^{\circ} \mathrm{C}$. The mixture was stirred at room temperature overnight. The precipitates were filtered out, and the solvent was removed. Purification by column chromatography using hexane/ethyl acetate (95/5) as eluent gave the product $\mathrm{H}_{2}\left(7.34 \mathrm{~g}, 80 \%\right.$ yield). ${ }^{1} \mathrm{H}-\mathrm{NMR}\left(500 \mathrm{MHz}, \mathrm{CDCl}_{3}, \delta\right)$ : 7.40-7.30 (5H, Ar- $\underline{H}), 5.21-5.06\left(3 \mathrm{H}, \mathrm{Ar}-\mathrm{CH}_{2} \mathrm{O}\right.$ and $\left.\mathrm{OC} \underline{H}\left(\mathrm{CH}_{2}\right) \mathrm{CO}\right), 4.30\left(1 \mathrm{H},\left(\mathrm{CH}_{3}\right)_{3} \mathrm{Si}\left(\mathrm{CH}_{3}\right)_{2} \mathrm{OC} \underline{H}\left(\mathrm{CH}_{2}\right) \mathrm{CO}\right)$, 4.10-4.00 (4H, $\left.\mathrm{OC}_{\underline{H}} \mathrm{CH}_{2} \mathrm{CH}_{2}\right), 2.53-2.34\left(4 \mathrm{H}, \mathrm{CHCH}_{2} \underline{\mathrm{CH}}_{2} \mathrm{CO}\right), 2.32-2.08$ (4H, $\left.\mathrm{CHC}_{2} \mathrm{CH}_{2} \mathrm{CO}\right), 1.60$ (4H, $\left.\mathrm{OCH}_{2} \underline{\mathrm{C}}_{2} \mathrm{CH}_{2} \mathrm{CH}_{2}\right), \quad 1.39-1.15 \quad\left(52 \mathrm{H}, \quad \mathrm{OCH}_{2} \mathrm{CH}_{2}\left(\mathrm{C}_{2}\right)_{13} \mathrm{CH}_{3}\right), \quad 0.94-0.82 \quad\left(15 \mathrm{H}, \quad \mathrm{CH}_{2} \mathrm{CH}_{2} \mathrm{C}_{3}\right.$ and $\left.\mathrm{Si}\left(\mathrm{CH}_{3}\right)_{2}\left(\mathrm{C}_{3}\right)_{3}\right), 0.02\left(6 \mathrm{H}, \mathrm{Si}\left(\mathrm{C}_{3}\right)_{2}\left(\mathrm{CH}_{3}\right)_{3}\right)$.

Synthesis of $\mathrm{H}_{2}-\mathrm{COOH} . \mathrm{H}_{2}(5.78 \mathrm{~g}, 6.20 \mathrm{mmol})$ was dissolved in ethyl acetate $(50 \mathrm{~mL})$. Palladium (400 mg, $10 \%$ on carbon) was then added. The mixture was stirred at room temperature under hydrogen atmosphere for 6h. The black suspension was filtered through a thick layer of celite. After removal of solvent, the product $\left(\mathrm{H}_{2}-\mathrm{COOH}\right)$ was obtained in high purity (above $\left.95 \%\right)$ and used directly.

Synthesis of $\mathrm{HO}-\mathrm{H}_{2} . \mathrm{H}_{2}(10.00 \mathrm{~g}, 10.74 \mathrm{mmol})$ was dissolved in anhydrous $\mathrm{CH}_{2} \mathrm{Cl}_{2} . \mathrm{BF}_{3}$-etherate $(10.0 \mathrm{~mL})$ was then slowly added at $0{ }^{\circ} \mathrm{C}$. The mixture was allowed to return to room temperature and was further stirred 
for $4 \mathrm{~h}$. The reaction was quenched by adding saturated $\mathrm{NaHCO}_{3}(150 \mathrm{~mL})$. The organic layer was separated and dried with $\mathrm{Na}_{2} \mathrm{SO}_{4}$. After removal of solvent, the crude product was purified by column chromatography using $n$-hexane/ethyl acetate (85/15) as eluent to give the pure material $\mathrm{HO}-\mathrm{H}_{2}\left(8.39 \mathrm{~g}, 96 \%\right.$ yield). ${ }^{1} \mathrm{H}-\mathrm{NMR}$ $\left(500 \mathrm{MHz}, \mathrm{CDCl}_{3}, \delta\right): 7.40-7.30(5 \mathrm{H}, \operatorname{Ar}-\underline{H}), 5.21-5.06\left(3 \mathrm{H}, \operatorname{Ar}-\underline{C} \underline{H_{2}} \mathrm{O}, \operatorname{OC} \underline{H}\left(\mathrm{CH}_{2}\right) \mathrm{CO}\right), 4.30(1 \mathrm{H}$, $\left.\mathrm{HOC} \underline{H}\left(\mathrm{CH}_{2}\right) \mathrm{CO}\right), \quad 4.10-4.00 \quad\left(4 \mathrm{H}, \quad \mathrm{OC}_{\underline{H}} \mathrm{CH}_{2} \mathrm{CH}_{2}\right), 2.53-2.34 \quad\left(4 \mathrm{H}, \mathrm{CHCH}_{2} \underline{\mathrm{C}}_{2} \mathrm{CO}\right), 2.32-2.08 \quad(4 \mathrm{H}$, $\left.\mathrm{CHC} \underline{H}_{2} \mathrm{CH}_{2} \mathrm{CO}\right), 1.60\left(4 \mathrm{H}, \mathrm{OCH}_{2} \underline{\mathrm{C}}_{2} \mathrm{CH}_{2} \mathrm{CH}_{2}\right), 1.39-1.15\left(52 \mathrm{H}, \mathrm{OCH}_{2} \mathrm{CH}_{2}\left(\mathrm{C}_{2}\right)_{13} \mathrm{CH}_{3}\right), 0.92-0.81(6 \mathrm{H}$, $\left.\mathrm{CH}_{2} \mathrm{CH}_{2} \mathrm{CH}_{3}\right)$.

Synthesis of $\mathrm{H}_{4} . \mathrm{HO}-\mathrm{H}_{2}(4.59 \mathrm{~g}, 5.61 \mathrm{mmol}), \mathrm{H}_{2}-\mathrm{COOH}(5.21 \mathrm{~g}, 6.20 \mathrm{mmol})$, and DPTS $(0.40 \mathrm{~g})$ were mixed together into a round bottom flask. After transferring into a glove box, the mixture was dissolved in dry DCM. DIC $(2.5 \mathrm{~mL})$ was then added slowly at $0{ }^{\circ} \mathrm{C}$. The mixture was stirred at room temperature overnight. The precipitates were filtered out, and the solvent was removed. Purification by column chromatography using hexane/ethyl acetate (10/90) as eluent gave the product $\mathrm{H}_{4}(8.80 \mathrm{~g}, 96 \%$ yield $) .{ }^{1} \mathrm{H}-\mathrm{NMR}\left(500 \mathrm{MHz}, \mathrm{CDCl}_{3}\right.$, $\delta): \quad 7.40-7.30 \quad(5 \mathrm{H}, \quad \operatorname{Ar}-\underline{H}), \quad 5.21-5.06 \quad\left(5 \mathrm{H}, \quad \mathrm{Ar}-\mathrm{C}_{2} \underline{H}_{2} \mathrm{O}\right.$ and $\left.\mathrm{OC} \underline{H}\left(\mathrm{CH}_{2}\right) \mathrm{CO}\right), \quad 4.30 \quad(1 \mathrm{H}$, $\left.\left(\mathrm{CH}_{3}\right)_{3} \mathrm{Si}\left(\mathrm{CH}_{3}\right)_{2} \mathrm{OC} \underline{H}\left(\mathrm{CH}_{2}\right) \mathrm{CO}\right), 4.10-4.00\left(8 \mathrm{H}, \mathrm{OCH}_{2} \mathrm{CH}_{2} \mathrm{CH}_{2}\right), 2.53-2.34\left(8 \mathrm{H}, \mathrm{CHCH}_{2} \mathrm{CH}_{2} \mathrm{CO}\right), 2.32-2.08$ (8H, $\left.\mathrm{CHC} \underline{H}_{2} \mathrm{CH}_{2} \mathrm{CO}\right), 1.60\left(8 \mathrm{H}, \mathrm{OCH}_{2} \mathrm{CH}_{2} \mathrm{CH}_{2} \mathrm{CH}_{2}\right), 1.39-1.15\left(104 \mathrm{H}, \mathrm{OCH}_{2} \mathrm{CH}_{2}\left(\mathrm{C}_{2}\right)_{13} \mathrm{CH}_{3}\right), 0.94-0.82(21 \mathrm{H}$, $\mathrm{CH}_{2} \mathrm{CH}_{2} \mathrm{C}_{3}$ and $\left.\mathrm{Si}\left(\mathrm{CH}_{3}\right)_{2}\left(\mathrm{C}_{3}\right)_{3}\right), 0.02\left(6 \mathrm{H}, \mathrm{Si}\left(\mathrm{C}_{\underline{H}_{3}}\right)_{2}\left(\mathrm{CH}_{3}\right)_{3}\right)$. MS (MALDI-ToF): [M+Na] $]^{+} \mathrm{Cal} 1662.76 ; \mathrm{Obs}$ 1662.08 .

\subsection{Discrete macromolecules with diverse geometries}

\subsection{1 $\mathrm{D}_{12}, \mathrm{D}_{14}$, and $\mathrm{D}_{16}$}

Synthesis of HO- $\mathrm{D}_{4} . \mathrm{D}_{4}(4.0 \mathrm{~g}, 2.82 \mathrm{mmol})$ was dissolved in anhydrous $\mathrm{CH}_{2} \mathrm{Cl}_{2}$. $\mathrm{BF}_{3}$-etherate $(4.0 \mathrm{~mL})$ was then slowly added at $0{ }^{\circ} \mathrm{C}$. The mixture was allowed to return to room temperature and was further stirred for $4 \mathrm{~h}$. The reaction was quenched by adding saturated $\mathrm{NaHCO}_{3}(100 \mathrm{~mL})$. The organic layer was separated and dried with $\mathrm{Na}_{2} \mathrm{SO}_{4}$. After removal of solvent, the crude product was purified by column chromatography using $n$-hexane/ethyl acetate (85/15) as eluent to give the pure material HO- $\mathrm{D}_{4}\left(3.35 \mathrm{~g}, 91 \%\right.$ yield). ${ }^{1} \mathrm{H}-\mathrm{NMR}(500$ $\left.\mathrm{MHz}, \mathrm{CDCl}_{3}, \delta\right): 7.40-7.30(5 \mathrm{H}, \mathrm{Ar}-\underline{H}), 5.21-5.06\left(5 \mathrm{H}, \mathrm{Ar}-\underline{C}_{2} \mathrm{O}, \mathrm{OC} \underline{H}\left(\mathrm{CH}_{2}\right) \mathrm{CO}\right), 4.30\left(1 \mathrm{H}, \mathrm{HOC} \underline{H}\left(\mathrm{CH}_{2}\right) \mathrm{CO}\right)$, 4.10-4.00 (8H, OC $\left.\underline{H}_{2} \mathrm{CH}_{2} \mathrm{CH}_{2}\right), 2.53-2.34\left(8 \mathrm{H}, \mathrm{CHCH}_{2} \underline{\mathrm{CH}}_{2} \mathrm{CO}\right), 2.32-2.08\left(8 \mathrm{H}, \mathrm{CHC}_{2} \mathrm{CH}_{2} \mathrm{CO}\right), 1.60(8 \mathrm{H}$, $\left.\mathrm{OCH}_{2} \mathrm{CH}_{2} \mathrm{CH}_{2} \mathrm{CH}_{2}\right), 1.39-1.15\left(72 \mathrm{H}, \mathrm{OCH}_{2} \mathrm{CH}_{2}\left(\mathrm{CH}_{2}\right)_{9} \mathrm{CH}_{3}\right), 0.92-0.81\left(12 \mathrm{H}, \mathrm{CH}_{2} \mathrm{CH}_{2} \mathrm{CH}_{3}\right)$.

Synthesis of $\mathrm{D}_{8} . \mathrm{HO}_{2} \mathrm{D}_{4}(4.24 \mathrm{~g}, 3.26 \mathrm{mmol}), \mathrm{D}_{4}-\mathrm{COOH}(5.22 \mathrm{~g}, 3.59 \mathrm{mmol})$, and DPTS $(0.25 \mathrm{~g})$ were mixed together into a round bottom flask. After transferring into a glove box, the mixture was dissolved in dry DCM. DIC $(5 \mathrm{~mL})$ was then added slowly at $0{ }^{\circ} \mathrm{C}$. The mixture was stirred at room temperature overnight. The 
precipitates were filtered out, and the solvent was removed. Purification by column chromatography using hexane/ethyl acetate $(85 / 15)$ as eluent gave the product $\mathrm{D}_{8}(8.23 \mathrm{~g}, 95 \%$ yield $) .{ }^{1} \mathrm{H}-\mathrm{NMR}\left(500 \mathrm{MHz}, \mathrm{CDCl}_{3}\right.$, $\delta): \quad 7.40-7.30 \quad(5 \mathrm{H}, \quad \mathrm{Ar}-\underline{H}), \quad 5.21-5.06 \quad\left(9 \mathrm{H}, \quad \mathrm{Ar}-\underline{C}_{2} \mathrm{O} \quad\right.$ and $\left.\mathrm{OC} \underline{H}\left(\mathrm{CH}_{2}\right) \mathrm{CO}\right), \quad 4.30 \quad(1 \mathrm{H}$, $\left.\left(\mathrm{CH}_{3}\right)_{3} \mathrm{Si}\left(\mathrm{CH}_{3}\right)_{2} \mathrm{OC} \underline{H}\left(\mathrm{CH}_{2}\right) \mathrm{CO}\right), 4.10-4.00\left(16 \mathrm{H}, \mathrm{OC}_{2} \mathrm{CH}_{2} \mathrm{CH}_{2}\right), 2.53-2.34\left(16 \mathrm{H}, \mathrm{CHCH}_{2} \underline{\mathrm{H}}_{2} \mathrm{CO}\right), 2.32-2.08$ $\left(16 \mathrm{H}, \mathrm{CHC} \underline{H}_{2} \mathrm{CH}_{2} \mathrm{CO}\right), 1.60\left(16 \mathrm{H}, \mathrm{OCH}_{2} \underline{\mathrm{C}}_{2} \mathrm{CH}_{2} \mathrm{CH}_{2}\right), 1.39-1.15\left(144 \mathrm{H}, \mathrm{OCH}_{2} \mathrm{CH}_{2}\left(\mathrm{C}_{2}\right)_{9} \mathrm{CH}_{3}\right), 0.94-0.82$ (39H, $\mathrm{CH}_{2} \mathrm{CH}_{2} \underline{\mathrm{C}}_{3}$ and $\left.\mathrm{Si}\left(\mathrm{CH}_{3}\right)_{2}\left(\mathrm{C}_{\underline{H}_{3}}\right)_{3}\right), 0.02\left(6 \mathrm{H}, \mathrm{Si}\left(\mathrm{C}_{3}\right)_{2}\left(\mathrm{CH}_{3}\right)_{3}\right)$. MS (MALDI-ToF): [M+Na] $]^{+} \mathrm{Cal}$ 2630.82; Obs 2631.58.

Synthesis of $\mathrm{D}_{\underline{8}}-\mathrm{COOH} . \mathrm{D}_{8}(3.05 \mathrm{~g}, 1.17 \mathrm{mmol})$ was dissolved in ethyl acetate $(30 \mathrm{~mL})$. Palladium $(200 \mathrm{mg}$, $10 \%$ on carbon) was then added. The mixture was stirred at room temperature under hydrogen atmosphere for 6h. The black suspension was filtered through a thick layer of celite. After removal of solvent, pure product $\left(\mathrm{D}_{8}-\mathrm{COOH}\right)$ was obtained in high purity (above $\left.95 \%\right)$ and used directly.

Synthesis of HO- $\underline{8}_{8} \mathrm{D}_{8}(1.60 \mathrm{~g}, 0.61 \mathrm{mmol})$ was dissolved in anhydrous $\mathrm{CH}_{2} \mathrm{Cl}_{2} . \mathrm{BF}_{3}$-etherate $(1.0 \mathrm{~mL})$ was then slowly added at $0{ }^{\circ} \mathrm{C}$. The mixture was allowed to return to room temperature and was further stirred for 4h. The reaction was quenched by adding saturated $\mathrm{NaHCO}_{3}(100 \mathrm{~mL})$. The organic layer was separated and dried with $\mathrm{Na}_{2} \mathrm{SO}_{4}$. After removal of solvent, the crude product was purified by column chromatography using $n$-hexane/ethyl acetate (85/15) as eluent to give the pure material HO- $\mathrm{D}_{8}\left(1.20 \mathrm{~g}, 79 \%\right.$ yield). ${ }^{1} \mathrm{H}-\mathrm{NMR}(500$ $\left.\mathrm{MHz}, \mathrm{CDCl}_{3}, \delta\right): 7.40-7.30(5 \mathrm{H}, \mathrm{Ar}-\underline{H}), 5.21-5.06\left(9 \mathrm{H}, \mathrm{Ar}-\underline{\mathrm{C}}_{2} \mathrm{O}, \mathrm{OC} \underline{H}\left(\mathrm{CH}_{2}\right) \mathrm{CO}\right), 4.30(1 \mathrm{H}, \mathrm{HOC} \underline{H}(\mathrm{CH}) \mathrm{CO})$, 4.10-4.00 (16H, OC $\left.\underline{H}_{2} \mathrm{CH}_{2} \mathrm{CH}_{2}\right), 2.53-2.34\left(16 \mathrm{H}, \mathrm{CHCH}_{2} \mathrm{CH}_{2} \mathrm{CO}\right), 2.32-2.08$ (16H, $\left.\mathrm{CHCH}_{2} \mathrm{CH}_{2} \mathrm{CO}\right), 1.60$ $\left(16 \mathrm{H}, \mathrm{OCH}_{2} \underline{\mathrm{C}}_{2} \mathrm{CH}_{2} \mathrm{CH}_{2}\right), 1.39-1.15\left(144 \mathrm{H}, \mathrm{OCH}_{2} \mathrm{CH}_{2}\left(\mathrm{C}_{2}\right)_{9} \mathrm{CH}_{3}\right), 0.92-0.81\left(24 \mathrm{H}, \mathrm{CH}_{2} \mathrm{CH}_{2} \underline{\mathrm{C}}_{3}\right)$.

Synthesis of $\mathrm{HO}-\mathrm{D}_{\underline{6}} . \mathrm{D}_{6}(4.0 \mathrm{~g}, 1.99 \mathrm{mmol})$ was dissolved in anhydrous $\mathrm{CH}_{2} \mathrm{Cl}_{2}$. $\mathrm{BF}_{3}$-etherate $(4.0 \mathrm{~mL})$ was then slowly added at $0{ }^{\circ} \mathrm{C}$. The mixture was allowed to return to room temperature and was further stirred for 4h. The reaction was quenched by adding saturated $\mathrm{NaHCO}_{3}(100 \mathrm{~mL})$. The organic layer was separated and dried with $\mathrm{Na}_{2} \mathrm{SO}_{4}$. After removal of solvent, the crude product was purified by column chromatography using $n$-hexane/ethyl acetate (85/15) as eluent to give the pure material $\mathrm{HO}-\mathrm{D}_{6}\left(3.30 \mathrm{~g}, 88 \%\right.$ yield). ${ }^{1} \mathrm{H}-\mathrm{NMR}(500$ $\left.\mathrm{MHz}, \mathrm{CDCl}_{3}, \delta\right):$ 7.40-7.30 (5H, Ar- $\left.\underline{H}\right), 5.21-5.06\left(7 \mathrm{H}, \mathrm{Ar}-\mathrm{C}_{2} \mathrm{O}, \mathrm{OC} \underline{H}\left(\mathrm{CH}_{2}\right) \mathrm{CO}\right), 4.30\left(1 \mathrm{H}, \mathrm{HOC} \underline{H}\left(\mathrm{CH}_{2}\right) \mathrm{CO}\right)$, 4.10-4.00 (12H, OC $\left.\underline{H}_{2} \mathrm{CH}_{2} \mathrm{CH}_{2}\right), 2.53-2.34\left(12 \mathrm{H}, \mathrm{CHCH}_{2} \underline{\mathrm{CH}}_{2} \mathrm{CO}\right), 2.32-2.08\left(12 \mathrm{H}, \mathrm{CHC}_{2} \mathrm{CH}_{2} \mathrm{CO}\right), 1.60$ $\left(12 \mathrm{H}, \mathrm{OCH}_{2} \underline{\mathrm{C}}_{2} \mathrm{CH}_{2} \mathrm{CH}_{2}\right), 1.39-1.15\left(108 \mathrm{H}, \mathrm{OCH}_{2} \mathrm{CH}_{2}\left(\mathrm{C}_{2}\right)_{9} \mathrm{CH}_{3}\right), 0.92-0.81\left(18 \mathrm{H}, \mathrm{CH}_{2} \mathrm{CH}_{2} \mathrm{C}_{3}\right)$.

Synthesis of $\mathrm{D}_{12}$. HO- $\mathrm{D}_{4}(0.35 \mathrm{~g}, 0.27 \mathrm{mmol}), \mathrm{D}_{8}-\mathrm{COOH}(0.70 \mathrm{~g}, 0.30 \mathrm{mmol})$, and DPTS $(0.15 \mathrm{~g})$ were mixed together into a round bottom flask. After transferring into a glove box, the mixture was dissolved in dry DCM. DIC $(1 \mathrm{~mL})$ was then added slowly at $0{ }^{\circ} \mathrm{C}$. The mixture was stirred at room temperature overnight. The 
precipitates were filtered out, and the solvent was removed. Purification by column chromatography using hexane/ethyl acetate $(85 / 15)$ as eluent gave the product $\mathrm{D}_{12}(0.94 \mathrm{~g}, 93 \%$ yield $) .{ }^{1} \mathrm{H}-\mathrm{NMR}\left(500 \mathrm{MHz}, \mathrm{CDCl}_{3}\right.$, $\delta): \quad 7.40-7.30 \quad(5 \mathrm{H}, \quad \operatorname{Ar}-\underline{H}), \quad 5.21-5.06 \quad\left(13 \mathrm{H}, \quad \operatorname{Ar}-\mathrm{C}_{2} \mathrm{O} \quad\right.$ and $\left.\mathrm{OC} \underline{H}\left(\mathrm{CH}_{2}\right) \mathrm{CO}\right), \quad 4.30 \quad(1 \mathrm{H}$, $\left.\left(\mathrm{CH}_{3}\right)_{3} \mathrm{Si}\left(\mathrm{CH}_{3}\right)_{2} \mathrm{OC} \underline{H}\left(\mathrm{CH}_{2}\right) \mathrm{CO}\right), 4.10-4.00\left(24 \mathrm{H}, \mathrm{OC}_{2} \mathrm{CH}_{2} \mathrm{CH}_{2}\right)$, 2.53-2.34 (24H, $\left.\mathrm{CHCH}_{2} \underline{\mathrm{H}}_{2} \mathrm{CO}\right), 2.32-2.08$ (24H, CHC $\left.\underline{H}_{2} \mathrm{CH}_{2} \mathrm{CO}\right), 1.60\left(24 \mathrm{H}, \mathrm{OCH}_{2} \underline{\mathrm{C}}_{2} \mathrm{CH}_{2} \mathrm{CH}_{2}\right), 1.39-1.15\left(216 \mathrm{H}, \mathrm{OCH}_{2} \mathrm{CH}_{2}\left(\mathrm{C}_{2}\right)_{9} \mathrm{CH}_{3}\right), 0.94-0.82$ $\left(45 \mathrm{H}, \mathrm{CH}_{2} \mathrm{CH}_{2} \underline{\mathrm{C}}_{3}\right.$ and $\left.\mathrm{Si}\left(\mathrm{CH}_{3}\right)_{2}\left(\mathrm{C}_{\underline{H}_{3}}\right)_{3}\right), 0.02\left(6 \mathrm{H}, \mathrm{Si}\left(\mathrm{C}_{3}\right)_{2}\left(\mathrm{CH}_{3}\right)_{3}\right) . \mathrm{MS}$ (MALDI-ToF): [M+Na] $]^{+} \mathrm{Cal}$ 3823.66; Obs 3823.41.

Synthesis of $\mathrm{D}_{\underline{14}} . \mathrm{HO}-\mathrm{D}_{6}(0.45 \mathrm{~g}, 0.24 \mathrm{mmol}), \mathrm{D}_{8}-\mathrm{COOH}(0.66 \mathrm{~g}, 0.26 \mathrm{mmol})$, and DPTS $(0.15 \mathrm{~g})$ were mixed together into a round bottom flask. After transferring into a glove box, the mixture was dissolved in dry DCM. DIC $(1 \mathrm{~mL})$ was then added slowly at $0{ }^{\circ} \mathrm{C}$. The mixture was stirred at room temperature overnight. The precipitates were filtered out, and the solvent was removed. Purification by column chromatography using hexane/ethyl acetate $(85 / 15)$ as eluent gave the product $\mathrm{D}_{14}(0.94 \mathrm{~g}, 89 \%$ yield $) .{ }^{1} \mathrm{H}-\mathrm{NMR}\left(500 \mathrm{MHz}, \mathrm{CDCl}_{3}\right.$, $\delta): \quad 7.40-7.30 \quad(5 \mathrm{H}, \quad \operatorname{Ar}-\underline{H}), \quad 5.21-5.06 \quad\left(15 \mathrm{H}, \quad \operatorname{Ar}-\underline{C}_{2} \mathrm{O} \quad\right.$ and $\left.\mathrm{OC} \underline{H}\left(\mathrm{CH}_{2}\right) \mathrm{CO}\right), \quad 4.30 \quad(1 \mathrm{H}$, $\left.\left(\mathrm{CH}_{3}\right)_{3} \mathrm{Si}\left(\mathrm{CH}_{3}\right)_{2} \mathrm{OC} \underline{H}\left(\mathrm{CH}_{2}\right) \mathrm{CO}\right), 4.10-4.00\left(28 \mathrm{H}, \mathrm{OC}_{2} \mathrm{CH}_{2} \mathrm{CH}_{2}\right), 2.53-2.34\left(28 \mathrm{H}, \mathrm{CHCH}_{2} \mathrm{C} \underline{H}_{2} \mathrm{CO}\right), 2.32-2.08$ (28H, CHC $\left.\underline{H}_{2} \mathrm{CH}_{2} \mathrm{CO}\right), 1.60\left(28 \mathrm{H}, \mathrm{OCH}_{2} \mathrm{CH}_{2} \mathrm{CH}_{2} \mathrm{CH}_{2}\right), 1.39-1.15\left(252 \mathrm{H}, \mathrm{OCH}_{2} \mathrm{CH}_{2}\left(\mathrm{CH}_{2}\right)_{9} \mathrm{CH}_{3}\right), 0.94-0.82$ $\left(51 \mathrm{H}, \mathrm{CH}_{2} \mathrm{CH}_{2} \mathrm{CH}_{3}\right.$ and $\left.\mathrm{Si}\left(\mathrm{CH}_{3}\right)_{2}\left(\mathrm{C}_{3}\right)_{3}\right), 0.02\left(6 \mathrm{H}, \mathrm{Si}\left(\mathrm{CH}_{3}\right)_{2}\left(\mathrm{CH}_{3}\right)_{3}\right)$. MS (MALDI-ToF): [M+Na] ${ }^{+} \mathrm{Cal}$ 4420.08; Obs 4420.02.

Synthesis of $\mathrm{D}_{16 .}$ HO- $\mathrm{D}_{8}(0.97 \mathrm{~g}, 0.39 \mathrm{mmol}), \mathrm{D}_{8}-\mathrm{COOH}(1.03 \mathrm{~g}, 0.41 \mathrm{mmol})$, and DPTS $(0.15 \mathrm{~g})$ were mixed together into a round bottom flask. After transferring into a glove box, the mixture was dissolved in dry DCM. DIC $(1 \mathrm{~mL})$ was then added slowly at $0{ }^{\circ} \mathrm{C}$. The mixture was stirred at room temperature overnight. The precipitates were filtered out, and the solvent was removed. Purification by column chromatography using hexane/ethyl acetate $(85 / 15)$ as eluent gave the product $\mathrm{D}_{16}(1.60 \mathrm{~g}, 80 \%$ yield $) .{ }^{1} \mathrm{H}-\mathrm{NMR}\left(500 \mathrm{MHz}, \mathrm{CDCl}_{3}\right.$,

$\delta): \quad 7.40-7.30 \quad(5 \mathrm{H}, \quad \operatorname{Ar}-\underline{H}), \quad 5.21-5.06 \quad\left(17 \mathrm{H}, \quad \operatorname{Ar}-\underline{C}_{2} \mathrm{O} \quad\right.$ and $\left.\mathrm{OC} \underline{H}\left(\mathrm{CH}_{2}\right) \mathrm{CO}\right), \quad 4.30 \quad(1 \mathrm{H}$, $\left.\left(\mathrm{CH}_{3}\right)_{3} \mathrm{Si}\left(\mathrm{CH}_{3}\right)_{2} \mathrm{OC} \underline{H}\left(\mathrm{CH}_{2}\right) \mathrm{CO}\right), 4.10-4.00\left(32 \mathrm{H}, \mathrm{OC}_{2} \mathrm{CH}_{2} \mathrm{CH}_{2}\right), 2.53-2.34\left(32 \mathrm{H}, \mathrm{CHCH}_{2} \underline{\mathrm{H}}_{2} \mathrm{CO}\right), 2.32-2.08$ (32H, CHCH $\left.\mathrm{CH}_{2} \mathrm{CO}\right), 1.60\left(32 \mathrm{H}, \mathrm{OCH}_{2} \mathrm{CH}_{2} \mathrm{CH}_{2} \mathrm{CH}_{2}\right), 1.39-1.15\left(288 \mathrm{H}, \mathrm{OCH}_{2} \mathrm{CH}_{2}\left(\mathrm{CH}_{2}\right)_{9} \mathrm{CH}_{3}\right), 0.94-0.82$ $\left(57 \mathrm{H}, \mathrm{CH}_{2} \mathrm{CH}_{2} \underline{\mathrm{H}}_{3}\right.$ and $\left.\mathrm{Si}\left(\mathrm{CH}_{3}\right)_{2}\left(\mathrm{C}_{3}\right)_{3}\right), 0.02\left(6 \mathrm{H}, \mathrm{Si}\left(\mathrm{C}_{H_{3}}\right)_{2}\left(\mathrm{CH}_{3}\right)_{3}\right) . \mathrm{MS}$ (MALDI-ToF): [M+Na] ${ }^{+} \mathrm{Cal}$ 5016.50; Obs 5016.44.

\subsection{2 $\mathrm{H}_{4} \mathrm{D}_{4} \mathrm{O}_{4} \mathrm{~B}_{4}$}

Synthesis of $\mathrm{H}_{4}-\mathrm{COOH} . \mathrm{H}_{4}(5.78 \mathrm{~g}, 6.20 \mathrm{mmol})$ was dissolved in ethyl acetate $(60 \mathrm{~mL})$. Palladium (400 mg, 
$10 \%$ on carbon) was then added. The mixture was stirred at room temperature under hydrogen atmosphere for 6h. The black suspension was filtered through a thick layer of celite. After removal of solvent, the product $\left(\mathrm{H}_{4}-\mathrm{COOH}\right)$ was obtained in high purity (above $\left.95 \%\right)$ and used directly.

Synthesis of $\mathrm{H}_{4} \underline{\mathrm{D}}_{4}=\mathrm{H}_{4}-\mathrm{COOH}(1.00 \mathrm{~g}, 0.61 \mathrm{mmol})$, HO-D4 (0.72 g, $\left.0.55 \mathrm{mmol}\right)$, and DPTS (0.75 g) were mixed together. After transferring into a glove box, the mixture was dissolved in dry DCM. DIC (0.5 mL) was then added slowly at $0{ }^{\circ} \mathrm{C}$. The mixture was stirred at room temperature overnight. The precipitates were filtered out, and the solvent was removed. Purification by column chromatography using hexane/ethyl acetate (85/15) as eluent gave the product $\mathrm{H}_{4} \mathrm{D}_{4}\left(1.48 \mathrm{~g}, 95 \%\right.$ yield). ${ }^{1} \mathrm{H}-\mathrm{NMR}\left(500 \mathrm{MHz}, \mathrm{CDCl}_{3}, \delta\right): 7.40-7.30(5 \mathrm{H}$, $\operatorname{Ar}-\underline{H}), 5.21-5.06\left(9 \mathrm{H}, \mathrm{Ar}-\mathrm{CH}_{2} \mathrm{O}\right.$ and $\left.\mathrm{OC} \underline{H}\left(\mathrm{CH}_{2}\right) \mathrm{CO}\right), 4.30\left(1 \mathrm{H},\left(\mathrm{CH}_{3}\right)_{3} \mathrm{Si}\left(\mathrm{CH}_{3}\right)_{2} \mathrm{OC} \underline{H}\left(\mathrm{CH}_{2}\right) \mathrm{CO}\right), 4.10-4.00$ $\left(16 \mathrm{H}, \mathrm{OCH}_{2} \mathrm{CH}_{2} \mathrm{CH}_{2}\right), 2.53-2.34\left(16 \mathrm{H}, \mathrm{CHCH}_{2} \mathrm{CH}_{2} \mathrm{CO}\right), 2.32-2.08\left(16 \mathrm{H}, \mathrm{CHCH}_{2} \mathrm{CH}_{2} \mathrm{CO}\right), 1.60$ (16H, $\left.\mathrm{OCH}_{2} \mathrm{CH}_{2} \mathrm{CH}_{2} \mathrm{CH}_{2}\right), 1.39-1.15$ (176H, $\mathrm{OCH}_{2} \mathrm{CH}_{2}\left(\mathrm{C}_{2}\right)_{13} \mathrm{CH}_{3}$ and $\left.\mathrm{OCH}_{2} \mathrm{CH}_{2}\left(\mathrm{C}_{2}\right)_{9} \mathrm{CH}_{3}\right), 0.94-0.82$ (33H, $\mathrm{CH}_{2} \mathrm{CH}_{2} \underline{\mathrm{CH}}_{3}$ and $\left.\mathrm{Si}\left(\mathrm{CH}_{3}\right)_{2}\left(\mathrm{C}_{3}\right)_{3}\right), 0.02\left(6 \mathrm{H}, \mathrm{Si}\left(\mathrm{C}_{\mathrm{H}_{3}}\right)_{2}\left(\mathrm{CH}_{3}\right)_{3}\right)$. MS (MALDI-ToF): [M+Na] ${ }^{+} \mathrm{Cal} 2855.60 ; \mathrm{Obs}$ 2854.90 .

Synthesis of $\mathrm{H}_{4} \underline{\mathrm{D}}_{4}-\mathrm{COOH} . \mathrm{H}_{4} \mathrm{D}_{4}(1.20 \mathrm{~g}, 0.42 \mathrm{mmol})$ was dissolved in ethyl acetate $(20 \mathrm{~mL})$. Palladium (50 $\mathrm{mg}, 10 \%$ on carbon) was then added. The mixture was stirred at room temperature under hydrogen atmosphere for $6 \mathrm{~h}$. The black suspension was filtered through a thick layer of celite. After removal of solvent, the product $\left(\mathrm{H}_{4} \mathrm{D}_{4}-\mathrm{COOH}\right)$ was obtained in high purity (above $\left.95 \%\right)$ and used directly.

Synthesis of $\mathrm{HO}-\mathrm{O}_{4} . \mathrm{O}_{4}(5.0 \mathrm{~g}, 4.19 \mathrm{mmol})$ was dissolved in anhydrous $\mathrm{CH}_{2} \mathrm{Cl}_{2}$. $\mathrm{BF}_{3}$-etherate $(5.0 \mathrm{~mL})$ was then slowly added at $0{ }^{\circ} \mathrm{C}$. The mixture was allowed to return to room temperature and was further stirred for 4h. The reaction was quenched by adding saturated $\mathrm{NaHCO}_{3}(100 \mathrm{~mL})$. The organic layer was separated and dried with $\mathrm{Na}_{2} \mathrm{SO}_{4}$. After removal of solvent, the crude product was purified by column chromatography using $n$-hexane/ethyl acetate (85/15) as eluent to give the pure material $\mathrm{HO}-\mathrm{O}_{4}\left(4.06 \mathrm{~g}, 90 \%\right.$ yield). ${ }^{1} \mathrm{H}-\mathrm{NMR}(500$ $\left.\mathrm{MHz}, \mathrm{CDCl}_{3}, \delta\right):$ 7.40-7.30 (5H, Ar- $\left.\underline{H}\right), 5.21-5.06\left(5 \mathrm{H}, \mathrm{Ar}-\mathrm{C}_{2} \mathrm{O}, \mathrm{OC} \underline{H}\left(\mathrm{CH}_{2}\right) \mathrm{CO}\right), 4.30\left(1 \mathrm{H}, \mathrm{HOC} \underline{H}\left(\mathrm{CH}_{2}\right) \mathrm{CO}\right)$, 4.10-4.00 (8H, OC $\left.\underline{H}_{2} \mathrm{CH}_{2} \mathrm{CH}_{2}\right), 2.53-2.34\left(8 \mathrm{H}, \mathrm{CHCH}_{2} \underline{\mathrm{H}}_{2} \mathrm{CO}\right), 2.32-2.08\left(8 \mathrm{H}, \mathrm{CHCH}_{2} \mathrm{CH}_{2} \mathrm{CO}\right), 1.60(8 \mathrm{H}$, $\left.\mathrm{OCH}_{2} \mathrm{C}_{2} \mathrm{CH}_{2} \mathrm{CH}_{2}\right), 1.39-1.15\left(40 \mathrm{H}, \mathrm{OCH}_{2} \mathrm{CH}_{2}\left(\mathrm{C}_{2}\right)_{5} \mathrm{CH}_{3}\right), 0.92-0.81\left(12 \mathrm{H}, \mathrm{CH}_{2} \mathrm{CH}_{2} \underline{\mathrm{C}}_{3}\right)$.

Synthesis of $\mathrm{H}_{4} \underline{\mathrm{D}}_{4} \underline{\mathrm{O}}_{4} . \mathrm{H}_{4} \mathrm{D}_{4}-\mathrm{COOH}(1.10 \mathrm{~g}, 0.40 \mathrm{mmol}), \mathrm{HO}-\mathrm{O} 4(0.40 \mathrm{~g}, 0.37 \mathrm{mmol})$, and DPTS $(0.20 \mathrm{~g})$ were mixed together. After transferring into a glove box, the mixture was dissolved in dry DCM. DIC (0.5 mL) was then added slowly at $0{ }^{\circ} \mathrm{C}$. The mixture was stirred at room temperature overnight. The precipitates were filtered out, and the solvent was removed. Purification by column chromatography using hexane/ethyl acetate (85/15) as eluent gave the product $\mathrm{H}_{4} \mathrm{D}_{4} \mathrm{O}_{4}\left(1.34 \mathrm{~g}, 95 \%\right.$ yield). ${ }^{1} \mathrm{H}-\mathrm{NMR}\left(500 \mathrm{MHz}, \mathrm{CDCl}_{3}, \delta\right): 7.40-7.30(5 \mathrm{H}$, 
$\operatorname{Ar}-\underline{H})$, 5.21-5.06 (13H, Ar- $\underline{H}_{2} \mathrm{O}$ and $\left.\mathrm{OC} \underline{H}\left(\mathrm{CH}_{2}\right) \mathrm{CO}\right), 4.30\left(1 \mathrm{H},\left(\mathrm{CH}_{3}\right)_{3} \mathrm{Si}\left(\mathrm{CH}_{3}\right)_{2} \mathrm{OC} \underline{H}\left(\mathrm{CH}_{2}\right) \mathrm{CO}\right), 4.10-4.00$ $\left(24 \mathrm{H}, \mathrm{OC}_{2} \mathrm{CH}_{2} \mathrm{CH}_{2}\right), 2.53-2.34\left(24 \mathrm{H}, \mathrm{CHCH}_{2} \underline{\mathrm{C}}_{2} \mathrm{CO}\right), 2.32-2.08\left(24 \mathrm{H}, \mathrm{CHC}_{2} \mathrm{CH}_{2} \mathrm{CO}\right), 1.60$ (24H, $\left.\mathrm{OCH}_{2} \mathrm{C}_{2} \mathrm{CH}_{2} \mathrm{CH}_{2}\right), 1.39-1.15\left(216 \mathrm{H}, \mathrm{OCH}_{2} \mathrm{CH}_{2}\left(\mathrm{CH}_{2}\right)_{13} \mathrm{CH}_{3}, \mathrm{OCH}_{2} \mathrm{CH}_{2}\left(\mathrm{CH}_{2}\right)_{9} \mathrm{CH}_{3}\right.$, and $\left.\mathrm{OCH}_{2} \mathrm{CH}_{2}\left(\mathrm{CH}_{2}\right)_{5} \mathrm{CH}_{3}\right)$, 0.94-0.82 (45H, $\mathrm{CH}_{2} \mathrm{CH}_{2} \underline{\mathrm{C}}_{3}$ and $\left.\mathrm{Si}\left(\mathrm{CH}_{3}\right)_{2}\left(\mathrm{C}_{3}\right)_{3}\right), 0.02\left(6 \mathrm{H}, \mathrm{Si}\left(\mathrm{C}_{3}\right)_{2}\left(\mathrm{CH}_{3}\right)_{3}\right) . \mathrm{MS}$ (MALDI-ToF): [M+Na] $]^{+}$ Cal 3824.20; Obs 3823.53.

Synthesis of $\mathrm{H}_{4} \underline{D}_{4} \underline{\mathrm{O}}_{4}-\mathrm{COOH} . \mathrm{H}_{4} \mathrm{D}_{4} \mathrm{O}_{4}(1.20 \mathrm{~g}, 0.32 \mathrm{mmol})$ was dissolved in ethyl acetate $(20 \mathrm{~mL})$. Palladium (50 mg, 10\% on carbon) was then added. The mixture was stirred at room temperature under hydrogen atmosphere for $6 \mathrm{~h}$. The black suspension was filtered through a thick layer of celite. After removal of solvent, the product $\left(\mathrm{H}_{4} \mathrm{D}_{4} \mathrm{O}_{4}-\mathrm{COOH}\right)$ was obtained in high purity (above $95 \%$ ) and used directly.

Synthesis of $\mathrm{H}_{4} \underline{D}_{4} \underline{\mathrm{O}}_{4} \underline{\mathrm{D}}_{4}=\mathrm{H}_{4} \mathrm{D}_{4} \mathrm{O}_{4}-\mathrm{COOH}(1.16 \mathrm{~g}, 0.15 \mathrm{mmol}), \mathrm{HO}-\mathrm{B}_{4}(0.24 \mathrm{~g}, 0.37 \mathrm{mmol})$, and DPTS $(0.10 \mathrm{~g})$ were mixed together. After transferring into a glove box, the mixture was dissolved in dry DCM. DIC (0.5 $\mathrm{mL}$ ) was then added slowly at $0{ }^{\circ} \mathrm{C}$. The mixture was stirred at room temperature overnight. The precipitates were filtered out, and the solvent was removed. Purification by column chromatography using hexane/ethyl acetate $(85 / 15)$ as eluent gave the product $\mathrm{H}_{4} \mathrm{D}_{4} \mathrm{O}_{4} \mathrm{~B}_{4}\left(1.20 \mathrm{~g}, 95 \%\right.$ yield). ${ }^{1} \mathrm{H}-\mathrm{NMR}\left(500 \mathrm{MHz}, \mathrm{CDCl}_{3}, \delta\right)$ :

7.40-7.30 (5H, Ar- $\underline{H}), \quad 5.21-5.06 \quad\left(17 \mathrm{H}, \quad \operatorname{Ar}-\underline{C}_{2} \mathrm{O} \quad\right.$ and $\left.\quad \mathrm{OC} \underline{H}\left(\mathrm{CH}_{2}\right) \mathrm{CO}\right), \quad 4.30 \quad(1 \mathrm{H}$, $\left.\left(\mathrm{CH}_{3}\right)_{3} \mathrm{Si}\left(\mathrm{CH}_{3}\right)_{2} \mathrm{OC} \underline{H}\left(\mathrm{CH}_{2}\right) \mathrm{CO}\right)$, 4.10-4.00 (32H, OCH $\left.\mathrm{H}_{2} \mathrm{CH}_{2} \mathrm{CH}_{2}\right)$, 2.53-2.34 (32H, $\left.\mathrm{CHCH}_{2} \mathrm{CH}_{2} \mathrm{CO}\right), 2.32-2.08$ $\left(32 \mathrm{H}, \quad \mathrm{CHC} \underline{H}_{2} \mathrm{CH}_{2} \mathrm{CO}\right), \quad 1.60 \quad\left(32 \mathrm{H}, \quad \mathrm{OCH}_{2} \underline{\mathrm{C}}_{2} \mathrm{CH}_{2} \mathrm{CH}_{2}\right), \quad 1.39-1.15 \quad\left(224 \mathrm{H}, \quad \mathrm{OCH}_{2} \mathrm{CH}_{2}\left(\mathrm{C}_{2}\right)_{13} \mathrm{CH}_{3}\right.$, $\mathrm{OCH}_{2} \mathrm{CH}_{2}\left(\mathrm{C}_{2}\right)_{9} \mathrm{CH}_{3}, \mathrm{OCH}_{2} \mathrm{CH}_{2}\left(\mathrm{C}_{2}\right)_{5} \mathrm{CH}_{3}$, and $\left.\mathrm{OCH}_{2} \mathrm{CH}_{2} \mathrm{C}_{2} \mathrm{CH}_{3}\right), 0.94-0.82\left(57 \mathrm{H}, \mathrm{CH}_{2} \mathrm{CH}_{2} \mathrm{C}_{3}\right.$ and $\left.\mathrm{Si}\left(\mathrm{CH}_{3}\right)_{2}\left(\mathrm{CH}_{3}\right)_{3}\right), 0.02\left(6 \mathrm{H}, \mathrm{Si}\left(\mathrm{CH}_{3}\right)_{2}\left(\mathrm{CH}_{3}\right)_{3}\right) . \mathrm{MS}$ (MALDI-ToF): [M+Na] $]^{+} \mathrm{Cal}$ 4568.56; Obs 4567.93.

\subsection{3 $\mathrm{B}_{4} \mathrm{O}_{4} \mathrm{D}_{4} \mathrm{H}_{4}$}

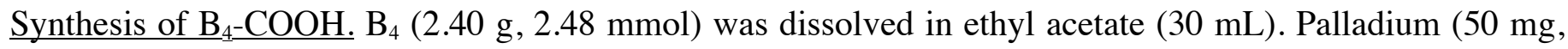
$10 \%$ on carbon) was then added. The mixture was stirred at room temperature under a hydrogen atmosphere for $6 \mathrm{~h}$. The black suspension was filtered through a thick layer of celite. After removal of solvent, the product $\left(\mathrm{B}_{4}-\mathrm{COOH}\right)$ was obtained in high purity (above $95 \%$ ) and used directly.

Synthesis of $\mathrm{B}_{4} \underline{\mathrm{O}}_{4 .} . \mathrm{B}_{4}-\mathrm{COOH}(1.00 \mathrm{~g}, 1.14 \mathrm{mmol}), \mathrm{HO}-\mathrm{O}_{4}(1.12 \mathrm{~g}, 1.04 \mathrm{mmol})$, and DPTS $(0.20 \mathrm{~g})$ were mixed together. After transferring into a glove box, the mixture was dissolved in dry DCM. DIC (0.5 mL) was then added slowly at $0{ }^{\circ} \mathrm{C}$. The mixture was stirred at room temperature overnight. The precipitates were filtered out, and the solvent was removed. Purification by column chromatography using hexane/ethyl acetate (85/15) as eluent gave the product $\mathrm{B}_{4} \mathrm{O}_{4}(1.89 \mathrm{~g}, 94 \%$ yield $) .{ }^{1} \mathrm{H}-\mathrm{NMR}\left(500 \mathrm{MHz}, \mathrm{CDCl}_{3}, \delta\right): 7.40-7.30(5 \mathrm{H}, \mathrm{Ar}-\underline{H})$, 5.21-5.06 (9H, Ar- $\underline{H}_{2} \mathrm{O}$ and $\left.\mathrm{OC} \underline{H}\left(\mathrm{CH}_{2}\right) \mathrm{CO}\right), 4.30\left(1 \mathrm{H},\left(\mathrm{CH}_{3}\right)_{3} \mathrm{Si}\left(\mathrm{CH}_{3}\right)_{2} \mathrm{OC} \underline{H}\left(\mathrm{CH}_{2}\right) \mathrm{CO}\right), 4.10-4.00(16 \mathrm{H}$, 
$\left.\mathrm{OC}_{2} \mathrm{CH}_{2} \mathrm{CH}_{2}\right), \quad 2.53-2.34 \quad\left(16 \mathrm{H}, \quad \mathrm{CHCH}_{2} \underline{\mathrm{C}}_{2} \mathrm{CO}\right), \quad 2.32-2.08 \quad\left(16 \mathrm{H}, \quad \mathrm{CHC} \underline{H}_{2} \mathrm{CH}_{2} \mathrm{CO}\right), \quad 1.60 \quad(16 \mathrm{H}$, $\left.\mathrm{OCH}_{2} \mathrm{CH}_{2} \mathrm{CH}_{2} \mathrm{CH}_{2}\right), \quad 1.39-1.15$ (48H, $\mathrm{OCH}_{2} \mathrm{CH}_{2} \mathrm{C}_{2} \mathrm{CH}_{3}$ and $\left.\mathrm{OCH}_{2} \mathrm{CH}_{2}\left(\mathrm{C}_{2}\right)_{5} \mathrm{CH}_{3}\right), \quad 0.94-0.82$ (33H, $\mathrm{CH}_{2} \mathrm{CH}_{2} \mathrm{CH}_{3}$ and $\left.\mathrm{Si}\left(\mathrm{CH}_{3}\right)_{2}\left(\mathrm{CH}_{3}\right)_{3}\right), 0.02\left(6 \mathrm{H}, \mathrm{Si}\left(\mathrm{C}_{3}\right)_{2}\left(\mathrm{CH}_{3}\right)_{3}\right)$. MS (MALDI-ToF): [M+Na] ${ }^{+} \mathrm{Cal}$ 1958.09; Obs 1957.95 .

Synthesis of $\mathrm{B}_{4} \underline{O}_{4}-\mathrm{COOH} . \mathrm{B}_{4} \mathrm{O}_{4}(1.50 \mathrm{~g}, 0.77 \mathrm{mmol})$ was dissolved in ethyl acetate $(20 \mathrm{~mL})$. Palladium $(50$ $\mathrm{mg}, 10 \%$ on carbon) was then added. The mixture was stirred at room temperature under hydrogen atmosphere for $6 \mathrm{~h}$. The black suspension was filtered through a thick layer of celite. After removal of solvent, the product $\left(\mathrm{B}_{4} \mathrm{O}_{4}-\mathrm{COOH}\right)$ was obtained in high purity (above $\left.95 \%\right)$ and used directly.

Synthesis of $\underline{B}_{4} \underline{\mathrm{O}}_{4} \underline{\mathrm{D}}_{4} . \mathrm{B}_{4} \mathrm{O}_{4}-\mathrm{COOH}(1.40 \mathrm{~g}, 0.76 \mathrm{mmol}), \mathrm{HO}-\mathrm{D}_{4}(0.90 \mathrm{~g}, 0.69 \mathrm{mmol})$, and DPTS (0.20 g) were mixed together. After transferring into a glove box, the mixture was dissolved in dry DCM. DIC (0.5 mL) was then added slowly at $0{ }^{\circ} \mathrm{C}$. The mixture was stirred at room temperature overnight. The precipitates were filtered out, and the solvent was removed. Purification by column chromatography using hexane/ethyl acetate $(85 / 15)$ as eluent gave the product $\mathrm{B}_{4} \mathrm{O}_{4} \mathrm{D}_{4}\left(1.99 \mathrm{~g}, 92 \%\right.$ yield). ${ }^{1} \mathrm{H}-\mathrm{NMR}\left(500 \mathrm{MHz}, \mathrm{CDCl}_{3}, \delta\right): 7.40-7.30(5 \mathrm{H}$, $\operatorname{Ar}-\underline{H}), 5.21-5.06\left(13 \mathrm{H}, \operatorname{Ar}-\underline{C}_{2} \mathrm{O}\right.$ and $\left.\mathrm{OC} \underline{H}\left(\mathrm{CH}_{2}\right) \mathrm{CO}\right), 4.30\left(1 \mathrm{H},\left(\mathrm{CH}_{3}\right)_{3} \mathrm{Si}\left(\mathrm{CH}_{3}\right)_{2} \mathrm{OC} \underline{H}\left(\mathrm{CH}_{2}\right) \mathrm{CO}\right), 4.10-4.00$ $\left(24 \mathrm{H}, \mathrm{OCH}_{2} \mathrm{CH}_{2} \mathrm{CH}_{2}\right), 2.53-2.34\left(24 \mathrm{H}, \mathrm{CHCH}_{2} \mathrm{CH}_{2} \mathrm{CO}\right), 2.32-2.08\left(24 \mathrm{H}, \mathrm{CHCH}_{2} \mathrm{CH}_{2} \mathrm{CO}\right), 1.60$ (24H, $\left.\mathrm{OCH}_{2} \mathrm{CH}_{2} \mathrm{CH}_{2} \mathrm{CH}_{2}\right), 1.39-1.15\left(120 \mathrm{H}, \mathrm{OCH}_{2} \mathrm{CH}_{2} \mathrm{CH}_{2} \mathrm{CH}_{3}, \mathrm{OCH}_{2} \mathrm{CH}_{2}\left(\mathrm{CH}_{2}\right)_{5} \mathrm{CH}_{3}\right.$, and $\left.\mathrm{OCH}_{2} \mathrm{CH}_{2}\left(\mathrm{CH}_{2}\right)_{9} \mathrm{CH}_{3}\right)$, 0.94-0.82 (45H, $\mathrm{CH}_{2} \mathrm{CH}_{2} \mathrm{C}_{3}$ and $\left.\mathrm{Si}\left(\mathrm{CH}_{3}\right)_{2}\left(\mathrm{C}_{3}\right)_{3}\right), 0.02$ (6H, $\left.\mathrm{Si}\left(\mathrm{C}_{\mathrm{H}_{3}}\right)_{2}\left(\mathrm{CH}_{3}\right)_{3}\right)$. MS (MALDI-ToF): [M+Na] $]^{+}$ Cal 3150.93; Obs 3150.75 .

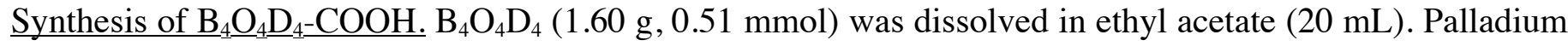
(50 mg, 10\% on carbon) was then added. The mixture was stirred at room temperature under hydrogen atmosphere for $6 \mathrm{~h}$. The black suspension was filtered through a thick layer of celite. After removal of solvent, the product $\left(\mathrm{B}_{4} \mathrm{O}_{4} \mathrm{D}_{4}-\mathrm{COOH}\right)$ was obtained in high purity (above $\left.95 \%\right)$ and used directly.

Synthesis of $\mathrm{HO}-\mathrm{H}_{4} . \mathrm{H}_{4}(4.0 \mathrm{~g}, 2.44 \mathrm{mmol})$ was dissolved in anhydrous $\mathrm{CH}_{2} \mathrm{Cl}_{2}$. $\mathrm{BF}_{3}$-etherate $(4.0 \mathrm{~mL})$ was then slowly added at $0{ }^{\circ} \mathrm{C}$. The mixture was allowed to return to room temperature and was further stirred for 4h. The reaction was quenched by adding saturated $\mathrm{NaHCO}_{3}(100 \mathrm{~mL})$. The organic layer was separated and dried with $\mathrm{Na}_{2} \mathrm{SO}_{4}$. After removal of solvent, the crude product was purified by column chromatography using $n$-hexane/ethyl acetate (85/15) as eluent to give the pure material $\mathrm{HO}-\mathrm{H}_{4}\left(3.30 \mathrm{~g}, 89 \%\right.$ yield). ${ }^{1} \mathrm{H}-\mathrm{NMR}(500$ $\left.\mathrm{MHz}, \mathrm{CDCl}_{3}, \delta\right):$ 7.40-7.30 (5H, Ar- $\left.\underline{H}\right), 5.21-5.06\left(5 \mathrm{H}, \mathrm{Ar}-\underline{\mathrm{C}}_{2} \mathrm{O}, \mathrm{OC} \underline{\mathrm{H}}\left(\mathrm{CH}_{2}\right) \mathrm{CO}\right), 4.30\left(1 \mathrm{H}, \mathrm{HOC} \underline{H}\left(\mathrm{CH}_{2}\right) \mathrm{CO}\right)$, 4.10-4.00 (8H, OC $\left.\underline{H}_{2} \mathrm{CH}_{2} \mathrm{CH}_{2}\right), 2.53-2.34\left(8 \mathrm{H}, \mathrm{CHCH}_{2} \mathrm{CH}_{2} \mathrm{CO}\right), 2.32-2.08\left(8 \mathrm{H}, \mathrm{CHCH}_{2} \mathrm{CH}_{2} \mathrm{CO}\right), 1.60(8 \mathrm{H}$, $\left.\mathrm{OCH}_{2} \mathrm{CH}_{2} \mathrm{CH}_{2} \mathrm{CH}_{2}\right)$, 1.39-1.15 (104H, OCH $\left.\mathrm{CH}_{2}\left(\mathrm{C}_{2}\right)_{13} \mathrm{CH}_{3}\right), 0.92-0.81\left(12 \mathrm{H}, \mathrm{CH}_{2} \mathrm{CH}_{2} \mathrm{C}_{3}\right)$. 


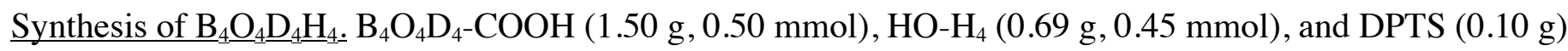
were mixed together. After transferring into a glove box, the mixture was dissolved in dry DCM. DIC (0.5 $\mathrm{mL}$ ) was then added slowly at $0{ }^{\circ} \mathrm{C}$. The mixture was stirred at room temperature overnight. The precipitates were filtered out, and the solvent was removed. Purification by column chromatography using hexane/ethyl acetate $(85 / 15)$ as eluent gave the product $\mathrm{B}_{4} \mathrm{O}_{4} \mathrm{D}_{4} \mathrm{H}_{4}\left(1.84 \mathrm{~g}, 90 \%\right.$ yield). ${ }^{1} \mathrm{H}-\mathrm{NMR}\left(500 \mathrm{MHz}, \mathrm{CDCl}_{3}, \delta\right)$ : 7.40-7.30 (5H, Ar- $\underline{H}), \quad 5.21-5.06 \quad\left(17 \mathrm{H}, \quad \operatorname{Ar}-\underline{\mathrm{C}}_{2} \mathrm{O} \quad\right.$ and $\left.\mathrm{OC} \underline{H}\left(\mathrm{CH}_{2}\right) \mathrm{CO}\right), \quad 4.30 \quad(1 \mathrm{H}$, $\left.\left(\mathrm{CH}_{3}\right)_{3} \mathrm{Si}\left(\mathrm{CH}_{3}\right)_{2} \mathrm{OC} \underline{H}\left(\mathrm{CH}_{2}\right) \mathrm{CO}\right)$, 4.10-4.00 (32H, OCH $\left.\mathrm{H}_{2} \mathrm{CH}_{2} \mathrm{CH}_{2}\right)$, 2.53-2.34 (32H, $\left.\mathrm{CHCH}_{2} \mathrm{CH}_{2} \mathrm{CO}\right), 2.32-2.08$ $\left(32 \mathrm{H}, \quad \mathrm{CHCH}_{2} \mathrm{CH}_{2} \mathrm{CO}\right), \quad 1.60 \quad\left(32 \mathrm{H}, \quad \mathrm{OCH}_{2} \underline{\mathrm{C}}_{2} \mathrm{CH}_{2} \mathrm{CH}_{2}\right), \quad 1.39-1.15 \quad\left(224 \mathrm{H}, \quad \mathrm{OCH}_{2} \mathrm{CH}_{2} \mathrm{C}_{2} \mathrm{CH}_{3}\right.$, $\mathrm{OCH}_{2} \mathrm{CH}_{2}\left(\mathrm{C}_{2}\right)_{5} \mathrm{CH}_{3}, \mathrm{OCH}_{2} \mathrm{CH}_{2}\left(\mathrm{C}_{2}\right)_{9} \mathrm{CH}_{3}$, and $\left.\mathrm{OCH}_{2} \mathrm{CH}_{2}\left(\mathrm{C}_{2}\right)_{13} \mathrm{CH}_{3}\right), 0.94-0.82\left(57 \mathrm{H}, \mathrm{CH}_{2} \mathrm{CH}_{2} \mathrm{C}_{3}\right.$ and $\left.\mathrm{Si}\left(\mathrm{CH}_{3}\right)_{2}\left(\mathrm{C}_{3}\right)_{3}\right), 0.02\left(6 \mathrm{H}, \mathrm{Si}\left(\mathrm{C}_{3}\right)_{2}\left(\mathrm{CH}_{3}\right)_{3}\right) . \mathrm{MS}$ (MALDI-ToF): [M+Na] $]^{+} \mathrm{Cal}$ 4568.56; Obs 4567.75 .

\subsection{4 $\mathrm{O}_{4} \mathrm{D}_{4} \mathrm{H}_{4}$}

Synthesis of $\mathrm{O}_{4}-\mathrm{COOH} . \mathrm{O}_{4}(4.10 \mathrm{~g}, 3.44 \mathrm{mmol})$ was dissolved in ethyl acetate $(50 \mathrm{~mL})$. Palladium $(200 \mathrm{mg}$, $10 \%$ on carbon) was then added. The mixture was stirred at room temperature under a hydrogen atmosphere for $6 \mathrm{~h}$. The black suspension was filtered through a thick layer of celite. After removal of solvent, the product $\left(\mathrm{O}_{4}-\mathrm{COOH}\right)$ was obtained in high purity (above $\left.95 \%\right)$ and used directly.

Synthesis of $\mathrm{O}_{4} \underline{\mathrm{D}}_{4=} \mathrm{O}_{4}-\mathrm{COOH}(1.00 \mathrm{~g}, 0.90 \mathrm{mmol}), \mathrm{HO}-\mathrm{D}_{4}(1.07 \mathrm{~g}, 0.82 \mathrm{mmol})$, and DPTS $(0.10 \mathrm{~g})$ were mixed together. After transferring into a glove box, the mixture was dissolved in dry DCM. DIC (0.5 mL) was then added slowly at $0{ }^{\circ} \mathrm{C}$. The mixture was stirred at room temperature overnight. The precipitates were filtered out, and the solvent was removed. Purification by column chromatography using hexane/ethyl acetate (85/15) as eluent gave the product $\mathrm{O}_{4} \mathrm{D}_{4}\left(1.84 \mathrm{~g}, 94 \%\right.$ yield). ${ }^{1} \mathrm{H}-\mathrm{NMR}\left(500 \mathrm{MHz}, \mathrm{CDCl}_{3}, \delta\right): 7.40-7.30(5 \mathrm{H}$, $\operatorname{Ar}-\underline{H})$, 5.21-5.06 (9H, Ar- $\underline{H}_{2} \mathrm{O}$ and $\left.\mathrm{OC} \underline{H}\left(\mathrm{CH}_{2}\right) \mathrm{CO}\right), 4.30\left(1 \mathrm{H},\left(\mathrm{CH}_{3}\right)_{3} \mathrm{Si}\left(\mathrm{CH}_{3}\right)_{2} \mathrm{OC} \underline{H}\left(\mathrm{CH}_{2}\right) \mathrm{CO}\right), 4.10-4.00$ $\left(16 \mathrm{H}, \mathrm{OCH}_{2} \mathrm{CH}_{2} \mathrm{CH}_{2}\right), 2.53-2.34\left(16 \mathrm{H}, \mathrm{CHCH}_{2} \mathrm{CH}_{2} \mathrm{CO}\right), 2.32-2.08\left(16 \mathrm{H}, \mathrm{CHCH}_{2} \mathrm{CH}_{2} \mathrm{CO}\right), 1.60$ (16H, $\left.\mathrm{OCH}_{2} \mathrm{CH}_{2} \mathrm{CH}_{2} \mathrm{CH}_{2}\right), 1.39-1.15\left(112 \mathrm{H}, \mathrm{OCH}_{2} \mathrm{CH}_{2}\left(\mathrm{CH}_{2}\right)_{5} \mathrm{CH}_{3}\right.$ and $\left.\mathrm{OCH}_{2} \mathrm{CH}_{2}\left(\mathrm{CH}_{2}\right)_{9} \mathrm{CH}_{3}\right), 0.94-0.82$ (33H, $\mathrm{CH}_{2} \mathrm{CH}_{2} \mathrm{C}_{3}$ and $\left.\mathrm{Si}\left(\mathrm{CH}_{3}\right)_{2}\left(\mathrm{C}_{3}\right)_{3}\right), 0.02\left(6 \mathrm{H}, \mathrm{Si}\left(\mathrm{C}_{\underline{H}_{3}}\right)_{2}\left(\mathrm{CH}_{3}\right)_{3}\right)$. MS (MALDI-ToF): [M+Na] $]^{+} \mathrm{Cal} 2406.61 ; \mathrm{Obs}$ 2406.42 .

Synthesis of $\mathrm{O}_{4} \underline{\mathrm{D}}_{4}-\mathrm{COOH} . \mathrm{O}_{4} \mathrm{D}_{4}(1.50 \mathrm{~g}, 0.63 \mathrm{mmol})$ was dissolved in ethyl acetate $(30 \mathrm{~mL})$. Palladium (50 $\mathrm{mg}, 10 \%$ on carbon) was then added. The mixture was stirred at room temperature under hydrogen atmosphere for $6 \mathrm{~h}$. The black suspension was filtered through a thick layer of celite. After removal of solvent, the product $\left(\mathrm{O}_{4} \mathrm{D}_{4}-\mathrm{COOH}\right)$ was obtained in high purity (above $\left.95 \%\right)$ and used directly.

Synthesis of $\mathrm{O}_{4} \underline{\mathrm{D}}_{4} \underline{\mathrm{H}}_{4} . \mathrm{O}_{4} \mathrm{D}_{4}-\mathrm{COOH}(1.44 \mathrm{~g}, 0.63 \mathrm{mmol}), \mathrm{HO}-\mathrm{H}_{4}(0.87 \mathrm{~g}, 0.57 \mathrm{mmol})$, and DPTS $(0.20 \mathrm{~g})$ were 
mixed together. After transferring into a glove box, the mixture was dissolved in dry DCM. DIC (0.5 mL) was then added slowly at $0{ }^{\circ} \mathrm{C}$. The mixture was stirred at room temperature overnight. The precipitates were filtered out, and the solvent was removed. Purification by column chromatography using hexane/ethyl acetate (85/15) as eluent gave the product $\mathrm{O}_{4} \mathrm{D}_{4} \mathrm{H}_{4}\left(2.08 \mathrm{~g}, 96 \%\right.$ yield). ${ }^{1} \mathrm{H}-\mathrm{NMR}\left(500 \mathrm{MHz}, \mathrm{CDCl}_{3}, \delta\right): 7.40-7.30(5 \mathrm{H}$, $\operatorname{Ar}-\underline{H}), 5.21-5.06\left(13 \mathrm{H}, \operatorname{Ar}-\underline{C}_{2} \mathrm{O}\right.$ and $\left.\mathrm{OC} \underline{H}\left(\mathrm{CH}_{2}\right) \mathrm{CO}\right), 4.30\left(1 \mathrm{H},\left(\mathrm{CH}_{3}\right)_{3} \mathrm{Si}\left(\mathrm{CH}_{3}\right)_{2} \mathrm{OC} \underline{H}\left(\mathrm{CH}_{2}\right) \mathrm{CO}\right), 4.10-4.00$ $\left(24 \mathrm{H}, \mathrm{OC}_{2} \mathrm{CH}_{2} \mathrm{CH}_{2}\right), 2.53-2.34\left(24 \mathrm{H}, \mathrm{CHCH}_{2} \underline{\mathrm{C}}_{2} \mathrm{CO}\right), 2.32-2.08\left(24 \mathrm{H}, \mathrm{CHC}_{2} \mathrm{CH}_{2} \mathrm{CO}\right), 1.60$ (24H, $\left.\mathrm{OCH}_{2} \mathrm{CH}_{2} \mathrm{CH}_{2} \mathrm{CH}_{2}\right), 1.39-1.15\left(120 \mathrm{H}, \mathrm{OCH}_{2} \mathrm{CH}_{2}\left(\mathrm{CH}_{2}\right)_{5} \mathrm{CH}_{3}, \mathrm{OCH}_{2} \mathrm{CH}_{2}\left(\mathrm{CH}_{2}\right)_{9} \mathrm{CH}_{3}\right.$, and $\left.\mathrm{OCH}_{2} \mathrm{CH}_{2}\left(\mathrm{CH}_{2}\right)_{13} \mathrm{CH}_{3}\right)$, 0.94-0.82 (45H, $\mathrm{CH}_{2} \mathrm{CH}_{2} \mathrm{C}_{3}$ and $\left.\mathrm{Si}\left(\mathrm{CH}_{3}\right)_{2}\left(\mathrm{C}_{3}\right)_{3}\right), 0.02\left(6 \mathrm{H}, \mathrm{Si}\left(\mathrm{C}_{3}\right)_{2}\left(\mathrm{CH}_{3}\right)_{3}\right)$. MS (MALDI-ToF): [M+Na] $]^{+}$ Cal 3823.71; Obs 3823.41.

\subsection{5 $\mathrm{H}_{4} \mathrm{D}_{6} \mathrm{O}_{4}$}

Synthesis of $\mathrm{H}_{4} \underline{\mathrm{D}}_{6} . \mathrm{H}_{4}-\mathrm{COOH}(1.00 \mathrm{~g}, 0.61 \mathrm{mmol}), \mathrm{HO}-\mathrm{D}_{6}(1.04 \mathrm{~g}, 0.55 \mathrm{mmol})$, and DPTS (0.25 g) were mixed together. After transferring into a glove box, the mixture was dissolved in dry DCM. DIC (0.5 mL) was then added slowly at $0{ }^{\circ} \mathrm{C}$. The mixture was stirred at room temperature overnight. The precipitates were filtered out, and the solvent was removed. Purification by column chromatography using hexane/ethyl acetate (85/15) as eluent gave the product $\mathrm{H}_{4} \mathrm{D}_{4}\left(1.68 \mathrm{~g}, 89 \%\right.$ yield). ${ }^{1} \mathrm{H}-\mathrm{NMR}\left(500 \mathrm{MHz}, \mathrm{CDCl}_{3}, \delta\right): 7.40-7.30(5 \mathrm{H}$, $\operatorname{Ar}-\underline{H}), 5.21-5.06\left(11 \mathrm{H}, \operatorname{Ar}-\mathrm{CH}_{2} \mathrm{O}\right.$ and $\left.\mathrm{OC} \underline{H}\left(\mathrm{CH}_{2}\right) \mathrm{CO}\right), 4.30\left(1 \mathrm{H},\left(\mathrm{CH}_{3}\right)_{3} \mathrm{Si}\left(\mathrm{CH}_{3}\right)_{2} \mathrm{OC} \underline{H}\left(\mathrm{CH}_{2}\right) \mathrm{CO}\right), 4.10-4.00$ $\left(20 \mathrm{H}, \mathrm{OC}_{2} \mathrm{CH}_{2} \mathrm{CH}_{2}\right), 2.53-2.34\left(20 \mathrm{H}, \mathrm{CHCH}_{2} \underline{\mathrm{CH}}_{2} \mathrm{CO}\right), 2.32-2.08\left(20 \mathrm{H}, \mathrm{CHCH}_{2} \mathrm{CH}_{2} \mathrm{CO}\right), 1.60$ (20H, $\left.\mathrm{OCH}_{2} \mathrm{CH}_{2} \mathrm{CH}_{2} \mathrm{CH}_{2}\right), 1.39-1.15\left(212 \mathrm{H}, \mathrm{OCH}_{2} \mathrm{CH}_{2}\left(\mathrm{C}_{2}\right)_{13} \mathrm{CH}_{3}\right.$ and $\left.\mathrm{OCH}_{2} \mathrm{CH}_{2}\left(\mathrm{CH}_{2}\right)_{9} \mathrm{CH}_{3}\right), 0.94-0.82$ (39H, $\mathrm{CH}_{2} \mathrm{CH}_{2} \mathrm{CH}_{3}$ and $\left.\mathrm{Si}\left(\mathrm{CH}_{3}\right)_{2}\left(\mathrm{CH}_{3}\right)_{3}\right), 0.02\left(6 \mathrm{H}, \mathrm{Si}\left(\mathrm{CH}_{3}\right)_{2}\left(\mathrm{CH}_{3}\right)_{3}\right)$. MS (MALDI-ToF): [M+Na] $]^{+} \mathrm{Cal} 3451.53 ; \mathrm{Obs}$ 3451.41 .

Synthesis of $\mathrm{H}_{4} \underline{\mathrm{D}_{6}} \underline{\mathrm{COOH}} . \mathrm{H}_{4} \mathrm{D}_{6}(1.50 \mathrm{~g}, 0.44 \mathrm{mmol})$ was dissolved in ethyl acetate $(30 \mathrm{~mL})$. Palladium (50 $\mathrm{mg}, 10 \%$ on carbon) was then added. The mixture was stirred at room temperature under hydrogen atmosphere for $6 \mathrm{~h}$. The black suspension was filtered through a thick layer of celite. After removal of solvent, the product $\left(\mathrm{H}_{4} \mathrm{D}_{6}-\mathrm{COOH}\right)$ was obtained in high purity (above $\left.95 \%\right)$ and used directly.

Synthesis of $\mathrm{H}_{4} \underline{\mathrm{D}}_{6} \mathrm{O}_{4} . \mathrm{H}_{4} \mathrm{D}_{6}-\mathrm{COOH}(1.34 \mathrm{~g}, 0.40 \mathrm{mmol}), \mathrm{HO}-\mathrm{O}_{4}(0.40 \mathrm{~g}, 0.37 \mathrm{mmol})$, and DPTS $(0.20 \mathrm{~g})$ were mixed together. After transferring into a glove box, the mixture was dissolved in dry DCM. DIC (0.5 mL) was then added slowly at $0{ }^{\circ} \mathrm{C}$. The mixture was stirred at room temperature overnight. The precipitates were filtered out, and the solvent was removed. Purification by column chromatography using hexane/ethyl acetate (85/15) as eluent gave the product $\mathrm{H}_{4} \mathrm{D}_{6} \mathrm{O}_{4}(1.44 \mathrm{~g}, 89 \%$ yield $) .{ }^{1} \mathrm{H}-\mathrm{NMR}\left(500 \mathrm{MHz}, \mathrm{CDCl}_{3}, \delta\right): 7.40-7.30(5 \mathrm{H}$, $\operatorname{Ar}-\underline{H}), 5.21-5.06\left(15 \mathrm{H}, \mathrm{Ar}-\underline{C}_{2} \mathrm{O}\right.$ and $\left.\mathrm{OC} \underline{H}\left(\mathrm{CH}_{2}\right) \mathrm{CO}\right), 4.30\left(1 \mathrm{H},\left(\mathrm{CH}_{3}\right)_{3} \mathrm{Si}\left(\mathrm{CH}_{3}\right)_{2} \mathrm{OC} \underline{H}\left(\mathrm{CH}_{2}\right) \mathrm{CO}\right), 4.10-4.00$ 
$\left(28 \mathrm{H}, \mathrm{OCH}_{2} \mathrm{CH}_{2} \mathrm{CH}_{2}\right), 2.53-2.34\left(28 \mathrm{H}, \mathrm{CHCH}_{2} \underline{\mathrm{CH}}_{2} \mathrm{CO}\right), 2.32-2.08\left(28 \mathrm{H}, \mathrm{CHCH}_{2} \mathrm{CH}_{2} \mathrm{CO}\right), 1.60$ (28H, $\left.\mathrm{OCH}_{2} \mathrm{C}_{2} \mathrm{CH}_{2} \mathrm{CH}_{2}\right), 1.39-1.15\left(252 \mathrm{H}, \mathrm{OCH}_{2} \mathrm{CH}_{2}\left(\mathrm{C}_{2}\right)_{13} \mathrm{CH}_{3}, \mathrm{OCH}_{2} \mathrm{CH}_{2}\left(\mathrm{C}_{2}\right)_{9} \mathrm{CH}_{3}\right.$, and $\left.\mathrm{OCH}_{2} \mathrm{CH}_{2}\left(\mathrm{C}_{2}\right)_{5} \mathrm{CH}_{3}\right)$, 0.94-0.82 (51H, $\mathrm{CH}_{2} \mathrm{CH}_{2} \mathrm{CH}_{3}$ and $\left.\mathrm{Si}\left(\mathrm{CH}_{3}\right)_{2}\left(\mathrm{CH}_{3}\right)_{3}\right), 0.02\left(6 \mathrm{H}, \mathrm{Si}\left(\mathrm{CH}_{3}\right)_{2}\left(\mathrm{CH}_{3}\right)_{3}\right)$. MS (MALDI-ToF): [M+Na $]^{+}$ Cal 4420.14; Obs 4420.02.

\subsection{6 $\mathrm{O}_{4} \mathrm{D}_{6} \mathrm{H}_{4}$}

Synthesis of $\mathrm{O}_{4} \underline{\mathrm{D}_{6}} \underline{\mathrm{O}} \mathrm{O}_{4}-\mathrm{COOH}(1.00 \mathrm{~g}, 0.90 \mathrm{mmol}), \mathrm{HO}-\mathrm{D}_{6}(1.56 \mathrm{~g}, 0.82 \mathrm{mmol})$, and DPTS $(0.10 \mathrm{~g})$ were mixed together. After transferring into a glove box, the mixture was dissolved in dry DCM. DIC (0.5 mL) was then added slowly at $0{ }^{\circ} \mathrm{C}$. The mixture was stirred at room temperature overnight. The precipitates were filtered out, and the solvent was removed. Purification by column chromatography using hexane/ethyl acetate $(85 / 15)$ as eluent gave the product $\mathrm{O}_{4} \mathrm{D}_{6}\left(2.04 \mathrm{~g}, 84 \%\right.$ yield). ${ }^{1} \mathrm{H}-\mathrm{NMR}\left(500 \mathrm{MHz}, \mathrm{CDCl}_{3}, \delta\right): 7.40-7.30(5 \mathrm{H}$, $\operatorname{Ar}-\underline{H}), 5.21-5.06\left(11 \mathrm{H}, \operatorname{Ar}-\mathrm{C}_{2} \mathrm{O}\right.$ and $\left.\mathrm{OC} \underline{H}\left(\mathrm{CH}_{2}\right) \mathrm{CO}\right), 4.30\left(1 \mathrm{H},\left(\mathrm{CH}_{3}\right)_{3} \mathrm{Si}\left(\mathrm{CH}_{3}\right)_{2} \mathrm{OC} \underline{H}\left(\mathrm{CH}_{2}\right) \mathrm{CO}\right), 4.10-4.00$ $\left(2 \mathrm{H}, \mathrm{OCH}_{2} \mathrm{CH}_{2} \mathrm{CH}_{2}\right), 2.53-2.34\left(20 \mathrm{H}, \mathrm{CHCH}_{2} \underline{\mathrm{C}}_{2} \mathrm{CO}\right), 2.32-2.08\left(20 \mathrm{H}, \mathrm{CHCH}_{2} \mathrm{CH}_{2} \mathrm{CO}\right), 1.60$ (20H, $\left.\mathrm{OCH}_{2} \mathrm{CH}_{2} \mathrm{CH}_{2} \mathrm{CH}_{2}\right), 1.39-1.15\left(148 \mathrm{H}, \mathrm{OCH}_{2} \mathrm{CH}_{2}\left(\mathrm{C}_{2}\right)_{5} \mathrm{CH}_{3}\right.$ and $\left.\mathrm{OCH}_{2} \mathrm{CH}_{2}\left(\mathrm{C}_{2}\right)_{9} \mathrm{CH}_{3}\right), 0.94-0.82$ (39H, $\mathrm{CH}_{2} \mathrm{CH}_{2} \underline{\mathrm{CH}}_{3}$ and $\left.\mathrm{Si}\left(\mathrm{CH}_{3}\right)_{2}\left(\mathrm{C}_{3}\right)_{3}\right), 0.02\left(6 \mathrm{H}, \mathrm{Si}\left(\mathrm{C}_{\mathrm{H}_{3}}\right)_{2}\left(\mathrm{CH}_{3}\right)_{3}\right)$. MS (MALDI-ToF): [M+Na] ${ }^{+} \mathrm{Cal}$ 3003.04; Obs 3002.78 .

$\underline{\text { Synthesis of } \mathrm{O}_{4}} \underline{\underline{D_{6}}} \underline{-\mathrm{COOH}} . \mathrm{O}_{4} \mathrm{D}_{4}(1.50 \mathrm{~g}, 0.50 \mathrm{mmol})$ was dissolved in ethyl acetate $(30 \mathrm{~mL})$. Palladium (50 $\mathrm{mg}, 10 \%$ on carbon) was then added. The mixture was stirred at room temperature under hydrogen atmosphere for $6 \mathrm{~h}$. The black suspension was filtered through a thick layer of celite. After removal of solvent, the product $\left(\mathrm{O}_{4} \mathrm{D}_{6}-\mathrm{COOH}\right)$ was obtained in high purity (above $\left.95 \%\right)$ and used directly.

Synthesis of $\mathrm{O}_{4} \underline{\mathrm{D}}_{6} \underline{\mathrm{H}}_{4} . \mathrm{O}_{4} \mathrm{D}_{6}-\mathrm{COOH}(1.45 \mathrm{~g}, 0.50 \mathrm{mmol}), \mathrm{HO}-\mathrm{H}_{4}(0.87 \mathrm{~g}, 0.57 \mathrm{mmol})$, and DPTS $(0.20 \mathrm{~g})$ were mixed together. After transferring into a glove box, the mixture was dissolved in dry DCM. DIC (0.5 mL) was then added slowly at $0{ }^{\circ} \mathrm{C}$. The mixture was stirred at room temperature overnight. The precipitates were filtered out, and the solvent was removed. Purification by column chromatography using hexane/ethyl acetate $(85 / 15)$ as eluent gave the product $\mathrm{O}_{4} \mathrm{D}_{6} \mathrm{H}_{4}(2.18 \mathrm{~g}, 87 \%$ yield $) .{ }^{1} \mathrm{H}-\mathrm{NMR}\left(500 \mathrm{MHz}, \mathrm{CDCl}_{3}, \delta\right): 7.40-7.30(5 \mathrm{H}$, $\operatorname{Ar}-\underline{H}), 5.21-5.06\left(15 \mathrm{H}, \mathrm{Ar}-\mathrm{C}_{2} \mathrm{O}\right.$ and $\left.\mathrm{OC} \underline{H}\left(\mathrm{CH}_{2}\right) \mathrm{CO}\right), 4.30\left(1 \mathrm{H},\left(\mathrm{CH}_{3}\right)_{3} \mathrm{Si}\left(\mathrm{CH}_{3}\right)_{2} \mathrm{OC} \underline{H}\left(\mathrm{CH}_{2}\right) \mathrm{CO}\right), 4.10-4.00$ $\left(28 \mathrm{H}, \mathrm{OC}_{2} \mathrm{CH}_{2} \mathrm{CH}_{2}\right), 2.53-2.34\left(28 \mathrm{H}, \mathrm{CHCH}_{2} \underline{\mathrm{H}}_{2} \mathrm{CO}\right), 2.32-2.08\left(28 \mathrm{H}, \mathrm{CHC}_{2} \mathrm{CH}_{2} \mathrm{CO}\right), 1.60$ (28H, $\left.\mathrm{OCH}_{2} \mathrm{C}_{2} \mathrm{CH}_{2} \mathrm{CH}_{2}\right), 1.39-1.15\left(252 \mathrm{H}, \mathrm{OCH}_{2} \mathrm{CH}_{2}\left(\mathrm{C}_{2}\right)_{5} \mathrm{CH}_{3}, \mathrm{OCH}_{2} \mathrm{CH}_{2}\left(\mathrm{C}_{2}\right)_{9} \mathrm{CH}_{3}\right.$, and $\left.\mathrm{OCH}_{2} \mathrm{CH}_{2}\left(\mathrm{C}_{2}\right)_{13} \mathrm{CH}_{3}\right)$, 0.94-0.82 (51H, $\mathrm{CH}_{2} \mathrm{CH}_{2} \mathrm{C}_{3}$ and $\left.\mathrm{Si}\left(\mathrm{CH}_{3}\right)_{2}\left(\mathrm{C}_{3}\right)_{3}\right), 0.02\left(6 \mathrm{H}, \mathrm{Si}\left(\mathrm{C}_{3}\right)_{2}\left(\mathrm{CH}_{3}\right)_{3}\right)$. MS (MALDI-ToF): [M+Na] $]^{+}$ Cal 4420.14; Obs 4420.03.

\subsection{7 $\mathrm{H}_{4} \mathrm{D}_{4} \mathrm{O}_{4} \mathrm{~B}_{4} \mathrm{H}_{4} \mathrm{D}_{4} \mathrm{O}_{4} \mathrm{~B}_{4}, \mathrm{H}_{4} \mathrm{D}_{4} \mathrm{O}_{4} \mathrm{~B}_{4} \mathrm{~B}_{4} \mathrm{O}_{4} \mathrm{D}_{4} \mathrm{H}_{4}$, and $\mathrm{B}_{4} \mathrm{O}_{4} \mathrm{D}_{4} \mathrm{H}_{8} \mathrm{H}_{4} \mathrm{D}_{4} \mathrm{O}_{4} \mathrm{~B}_{4}$}


Synthesis of $\mathrm{H}_{4} \underline{\mathrm{D}}_{4} \underline{\mathrm{O}}_{4} \underline{\mathrm{B}}_{4} \underline{-\mathrm{COOH}} . \mathrm{H}_{4} \mathrm{D}_{4} \mathrm{O}_{4} \mathrm{~B}_{4}(0.50 \mathrm{~g}, 0.11 \mathrm{mmol})$ was dissolved in ethyl acetate $(20 \mathrm{~mL})$. Palladium (50 mg, 10\% on carbon) was then added. The mixture was stirred at room temperature under a hydrogen atmosphere for $6 \mathrm{~h}$. The black suspension was filtered through a thick layer of celite. After removal of solvent, the product $\left(\mathrm{H}_{4} \mathrm{D}_{4} \mathrm{O}_{4} \mathrm{~B}_{4}-\mathrm{COOH}\right)$ was obtained in high purity (above $95 \%$ ) and used directly.

Synthesis of $\mathrm{B}_{4} \underline{\mathrm{O}}_{4} \underline{\mathrm{D}}_{4} \underline{\mathrm{H}}_{4}-\mathrm{COOH} . \mathrm{B}_{4} \mathrm{O}_{4} \mathrm{D}_{4} \mathrm{H}_{4}(0.50 \mathrm{~g}, 0.11 \mathrm{mmol})$ was dissolved in ethyl acetate $(20 \mathrm{~mL})$. Palladium (50 mg, 10\% on carbon) was then added. The mixture was stirred at room temperature under a hydrogen atmosphere for $6 \mathrm{~h}$. The black suspension was filtered through a thick layer of celite. After removal of solvent, the product $\left(\mathrm{B}_{4} \mathrm{O}_{4} \mathrm{D}_{4} \mathrm{H}_{4}-\mathrm{COOH}\right)$ was obtained in high purity (above $95 \%$ ) and used directly.

Synthesis of $\mathrm{HO}-\mathrm{H}_{4} \underline{\mathrm{D}}_{4} \underline{\mathrm{O}}_{4} \underline{\mathrm{B}}_{4} . \mathrm{H}_{4} \mathrm{D}_{4} \mathrm{O}_{4} \mathrm{~B}_{4}(0.50 \mathrm{~g}, 0.11 \mathrm{mmol})$ was dissolved in anhydrous $\mathrm{CH}_{2} \mathrm{Cl}_{2}$. $\mathrm{BF}_{3}$-etherate $(0.5 \mathrm{~mL})$ was then slowly added at $0{ }^{\circ} \mathrm{C}$. The mixture was allowed to return to room temperature and was further stirred for $4 \mathrm{~h}$. The reaction was quenched by adding saturated $\mathrm{NaHCO}_{3}(50 \mathrm{~mL})$. The organic layer was separated and dried with $\mathrm{Na}_{2} \mathrm{SO}_{4}$. After removal of solvent, the crude product was purified by column chromatography using $n$-hexane/ethyl acetate (85/15) as eluent to give the pure material $\mathrm{HO}-\mathrm{H}_{4} \mathrm{D}_{4} \mathrm{O}_{4} \mathrm{~B}_{4}(0.44$ $\mathrm{g}, 88 \%$ yield).

Synthesis of $\mathrm{HO}-\mathrm{B}_{4} \underline{\mathrm{O}}_{4} \underline{\mathrm{D}}_{4} \underline{\mathrm{H}_{4}} . \mathrm{B}_{4} \mathrm{O}_{4} \mathrm{D}_{4} \mathrm{H}_{4}(0.50 \mathrm{~g}, 0.11 \mathrm{mmol})$ was dissolved in anhydrous $\mathrm{CH}_{2} \mathrm{Cl}_{2}$. $\mathrm{BF}_{3}$-etherate $(0.5 \mathrm{~mL})$ was then slowly added at $0{ }^{\circ} \mathrm{C}$. The mixture was allowed to return to room temperature and was further stirred for $4 \mathrm{~h}$. The reaction was quenched by adding saturated $\mathrm{NaHCO}_{3}(50 \mathrm{~mL})$. The organic layer was separated and dried with $\mathrm{Na}_{2} \mathrm{SO}_{4}$. After removal of solvent, the crude product was purified by column chromatography using $n$-hexane/ethyl acetate $(85 / 15)$ as eluent to give the pure material $\mathrm{HO}-\mathrm{B}_{4} \mathrm{O}_{4} \mathrm{D}_{4} \mathrm{H}_{4}(0.42$ g, $86 \%$ yield).

Synthesis of $\mathrm{H}_{4} \underline{\mathrm{D}}_{4} \underline{\mathrm{O}}_{4} \underline{\mathrm{B}}_{4} \underline{\mathrm{H}}_{4} \underline{\mathrm{D}}_{4} \underline{\mathrm{O}}_{4} \underline{\mathrm{B}}_{4} \underline{\mathrm{H}}_{4} \mathrm{D}_{4} \mathrm{O}_{4} \mathrm{~B}_{4}-\mathrm{COOH}(0.20 \mathrm{~g}, 0.04 \mathrm{mmol}), \mathrm{HO}-\mathrm{H}_{4} \mathrm{D}_{4} \mathrm{O}_{4} \mathrm{~B}_{4}(0.18 \mathrm{~g}, 0.04 \mathrm{mmol})$, and DPTS $(0.20 \mathrm{~g})$ were mixed together. After transferring into a glove box, the mixture was dissolved in dry DCM. DIC $(0.5 \mathrm{~mL})$ was then added slowly at $0{ }^{\circ} \mathrm{C}$. The mixture was stirred at room temperature overnight. The precipitates were filtered out, and the solvent was removed. Purification by column chromatography using hexane/ethyl acetate (85/15) as eluent gave the desired product $\left(0.30 \mathrm{~g}, 83 \%\right.$ yield). ${ }^{1} \mathrm{H}-\mathrm{NMR}(500 \mathrm{MHz}$, $\left.\mathrm{CDCl}_{3}, \delta\right): \quad 7.40-7.30 \quad(5 \mathrm{H}, \quad \mathrm{Ar}-\underline{H}), \quad 5.21-5.06 \quad\left(33 \mathrm{H}, \quad \mathrm{Ar}-\mathrm{C} \underline{H_{2} \mathrm{O}}\right.$ and $\left.\mathrm{OC} \underline{H}\left(\mathrm{CH}_{2}\right) \mathrm{CO}\right), 4.30 \quad(1 \mathrm{H}$, $\left.\left(\mathrm{CH}_{3}\right)_{3} \mathrm{Si}\left(\mathrm{CH}_{3}\right)_{2} \mathrm{OC} \underline{H}\left(\mathrm{CH}_{2}\right) \mathrm{CO}\right), 4.10-4.00\left(64 \mathrm{H}, \mathrm{OC}_{2} \mathrm{CH}_{2} \mathrm{CH}_{2}\right), 2.53-2.34\left(64 \mathrm{H}, \mathrm{CHCH}_{2} \underline{\mathrm{H}}_{2} \mathrm{CO}\right), 2.32-2.08$ $\left(64 \mathrm{H}, \quad \mathrm{CHC} \underline{H}_{2} \mathrm{CH}_{2} \mathrm{CO}\right), \quad 1.60 \quad\left(64 \mathrm{H}, \quad \mathrm{OCH}_{2} \underline{\mathrm{C}}_{2} \mathrm{CH}_{2} \mathrm{CH}_{2}\right), \quad 1.39-1.15 \quad\left(448 \mathrm{H}, \quad \mathrm{OCH}_{2} \mathrm{CH}_{2}\left(\mathrm{C}_{2}\right)_{13} \mathrm{CH}_{3}\right.$, $\mathrm{OCH}_{2} \mathrm{CH}_{2}\left(\mathrm{C}_{2}\right)_{9} \mathrm{CH}_{3}, \mathrm{OCH}_{2} \mathrm{CH}_{2}\left(\mathrm{CH}_{2}\right)_{5} \mathrm{CH}_{3}$, and $\left.\mathrm{OCH}_{2} \mathrm{CH}_{2} \mathrm{CH}_{2} \mathrm{CH}_{3}\right), 0.94-0.82\left(105 \mathrm{H}, \mathrm{CH}_{2} \mathrm{CH}_{2} \mathrm{CH}_{3}\right.$ and $\left.\mathrm{Si}\left(\mathrm{CH}_{3}\right)_{2}\left(\mathrm{C}_{3}\right)_{3}\right), 0.02\left(6 \mathrm{H}, \mathrm{Si}\left(\mathrm{C}_{3}\right)_{2}\left(\mathrm{CH}_{3}\right)_{3}\right) . \mathrm{MS}$ (MALDI-ToF): [M+Na] ${ }^{+} \mathrm{Cal}$ 8891.00; Obs 8890.37. 


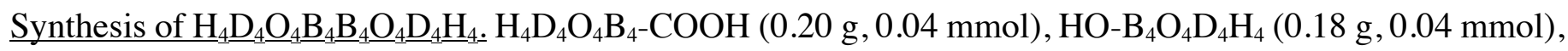
and DPTS $(0.20 \mathrm{~g})$ were mixed together. After transferring into a glove box, the mixture was dissolved in dry DCM. DIC $(0.5 \mathrm{~mL})$ was then added slowly at $0{ }^{\circ} \mathrm{C}$. The mixture was stirred at room temperature overnight. The precipitates were filtered out, and the solvent was removed. Purification by column chromatography using hexane/ethyl acetate $(85 / 15)$ as eluent gave the desired product $\left(0.31 \mathrm{~g}, 83 \%\right.$ yield). ${ }^{1} \mathrm{H}-\mathrm{NMR}(500 \mathrm{MHz}$, $\left.\mathrm{CDCl}_{3}, \delta\right): \quad 7.40-7.30 \quad(5 \mathrm{H}, \quad \mathrm{Ar}-\underline{H}), \quad 5.21-5.06 \quad\left(33 \mathrm{H}, \quad \mathrm{Ar}-\underline{\mathrm{C}}_{2} \mathrm{O}\right.$ and $\left.\mathrm{OC} \underline{H}\left(\mathrm{CH}_{2}\right) \mathrm{CO}\right), 4.30 \quad(1 \mathrm{H}$, $\left.\left(\mathrm{CH}_{3}\right)_{3} \mathrm{Si}\left(\mathrm{CH}_{3}\right)_{2} \mathrm{OC} \underline{H}\left(\mathrm{CH}_{2}\right) \mathrm{CO}\right)$, 4.10-4.00 (64H, OC $\left.\underline{H}_{2} \mathrm{CH}_{2} \mathrm{CH}_{2}\right)$, 2.53-2.34 (64H, $\left.\mathrm{CHCH}_{2} \mathrm{CH}_{2} \mathrm{CO}\right), 2.32-2.08$ $\left(64 \mathrm{H}, \quad \mathrm{CHC} \underline{H}_{2} \mathrm{CH}_{2} \mathrm{CO}\right), \quad 1.60 \quad\left(64 \mathrm{H}, \quad \mathrm{OCH}_{2} \underline{\mathrm{C}}_{2} \mathrm{CH}_{2} \mathrm{CH}_{2}\right), \quad 1.39-1.15 \quad\left(448 \mathrm{H}, \quad \mathrm{OCH}_{2} \mathrm{CH}_{2}\left(\mathrm{CH}_{2}\right)_{13} \mathrm{CH}_{3}\right.$, $\mathrm{OCH}_{2} \mathrm{CH}_{2}\left(\mathrm{C}_{2}\right)_{9} \mathrm{CH}_{3}, \mathrm{OCH}_{2} \mathrm{CH}_{2}\left(\mathrm{C}_{2}\right)_{5} \mathrm{CH}_{3}$, and $\left.\mathrm{OCH}_{2} \mathrm{CH}_{2} \mathrm{CH}_{2} \mathrm{CH}_{3}\right), 0.94-0.82\left(105 \mathrm{H}, \mathrm{CH}_{2} \mathrm{CH}_{2} \mathrm{C}_{3}\right.$ and $\left.\mathrm{Si}\left(\mathrm{CH}_{3}\right)_{2}\left(\mathrm{C}_{H_{3}}\right)_{3}\right), 0.02\left(6 \mathrm{H}, \mathrm{Si}\left(\mathrm{C}_{3}\right)_{2}\left(\mathrm{CH}_{3}\right)_{3}\right) . \mathrm{MS}$ (MALDI-ToF): [M+Na]+ Cal 8891.00; Obs 8890.41.

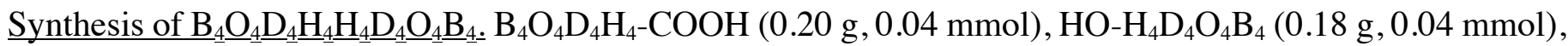
and DPTS $(0.20 \mathrm{~g})$ were mixed together. After transferring into a glove box, the mixture was dissolved in dry DCM. DIC $(0.5 \mathrm{~mL})$ was then added slowly at $0{ }^{\circ} \mathrm{C}$. The mixture was stirred at room temperature overnight. The precipitates were filtered out, and the solvent was removed. Purification by column chromatography using hexane/ethyl acetate $(85 / 15)$ as eluent gave the product $(0.29 \mathrm{~g}, 80 \%$ yield $) .{ }^{1} \mathrm{H}-\mathrm{NMR}\left(500 \mathrm{MHz}, \mathrm{CDCl}_{3}, \delta\right)$ :

7.40-7.30 (5H, Ar- $\underline{H}), \quad 5.21-5.06 \quad\left(33 \mathrm{H}, \quad \operatorname{Ar}-\underline{C}_{2} \mathrm{O} \quad\right.$ and $\left.\quad \mathrm{OC} \underline{H}\left(\mathrm{CH}_{2}\right) \mathrm{CO}\right), \quad 4.30 \quad(1 \mathrm{H}$, $\left.\left(\mathrm{CH}_{3}\right)_{3} \mathrm{Si}\left(\mathrm{CH}_{3}\right)_{2} \mathrm{OC} \underline{H}\left(\mathrm{CH}_{2}\right) \mathrm{CO}\right), 4.10-4.00\left(64 \mathrm{H}, \mathrm{OC}_{2} \mathrm{CH}_{2} \mathrm{CH}_{2}\right), 2.53-2.34\left(64 \mathrm{H}, \mathrm{CHCH}_{2} \underline{\mathrm{H}}_{2} \mathrm{CO}\right), 2.32-2.08$ $\left(64 \mathrm{H}, \quad \mathrm{CHC} \underline{H}_{2} \mathrm{CH}_{2} \mathrm{CO}\right), \quad 1.60 \quad\left(64 \mathrm{H}, \quad \mathrm{OCH}_{2} \mathrm{C}_{2} \mathrm{CH}_{2} \mathrm{CH}_{2}\right), \quad 1.39-1.15 \quad\left(448 \mathrm{H}, \quad \mathrm{OCH}_{2} \mathrm{CH}_{2}\left(\mathrm{CH}_{2}\right)_{13} \mathrm{CH}_{3}\right.$, $\mathrm{OCH}_{2} \mathrm{CH}_{2}\left(\mathrm{C}_{2}\right)_{9} \mathrm{CH}_{3}, \mathrm{OCH}_{2} \mathrm{CH}_{2}\left(\mathrm{C}_{2}\right)_{5} \mathrm{CH}_{3}$, and $\left.\mathrm{OCH}_{2} \mathrm{CH}_{2} \underline{\mathrm{H}}_{2} \mathrm{CH}_{3}\right), 0.94-0.82\left(105 \mathrm{H}, \mathrm{CH}_{2} \mathrm{CH}_{2} \mathrm{C}_{3}\right.$ and $\left.\mathrm{Si}\left(\mathrm{CH}_{3}\right)_{2}\left(\mathrm{C}_{3}\right)_{3}\right), 0.02\left(6 \mathrm{H}, \mathrm{Si}\left(\mathrm{C}_{3}\right)_{2}\left(\mathrm{CH}_{3}\right)_{3}\right) . \mathrm{MS}$ (MALDI-ToF): [M+Na] ${ }^{+} \mathrm{Cal}$ 8891 .00; Obs 8890.45.

\subsection{Discrete block copolymer}

\subsection{1 $\mathrm{D}_{12}-\mathrm{L}_{m}$ and $\mathrm{D}_{14}-\mathrm{L}_{m}$}

Synthesis of $\mathrm{D}_{12}$-COOH. $\mathrm{D}_{12}(500 \mathrm{mg})$ was dissolved in ethyl acetate $(20 \mathrm{~mL})$. Palladium (30 mg, $10 \%$ on carbon) was then added. The mixture was stirred at room temperature under hydrogen atmosphere for $6 \mathrm{~h}$. The black suspension was filtered through a thick layer of celite. After removal of solvent, the product (DGA ${ }_{12^{-}}$ $\mathrm{COOH}$ ) was obtained in high purity (above 95\%) and used directly.

General Procedure for Synthesis of HO-oLA $\mathrm{LA}_{\underline{m}} \mathrm{LA}_{24}(500.0 \mathrm{mg})$ was dissolved in anhydrous $\mathrm{CH}_{2} \mathrm{Cl}_{2}$. BF3etherate $(1.0 \mathrm{~mL})$ was then slowly added at $0{ }^{\circ} \mathrm{C}$. The mixture was allowed to return to room temperature and was further stirred for $4 \mathrm{~h}$. The reaction was quenched by adding saturated $\mathrm{NaHCO}_{3}(200 \mathrm{~mL})$. The organic layer was separated and dried with $\mathrm{Na}_{2} \mathrm{SO}_{4}$. After removal of solvent, the crude product was purified by 
column chromatography using $n$-hexane/ethyl acetate (40/60) as eluent to give the pure material $\mathrm{HO}-\mathrm{L}_{24}$ (424.0 mg, 90\% yield).

General Procedure for Synthesis of $\mathrm{D}_{12}-\mathrm{L}_{\underline{m}} . \mathrm{HO}-\mathrm{L}_{24}(40.0 \mathrm{mg}), \mathrm{D}_{12}-\mathrm{COOH}(80.0 \mathrm{mg})$, and DPTS (100.0 mg) were mixed together into a round bottom flask. After transferring into a glove box, the mixture was dissolved in dry DCM. DIC $(0.50 \mathrm{~mL})$ was then added slowly at $0{ }^{\circ} \mathrm{C}$. The mixture was stirred at room temperature overnight. The solvent was removed, and the obtained crude product was purified by recycling preparative SEC, giving pure product $\mathrm{D}_{12}-\mathrm{L}_{24}\left(50 \mathrm{mg}, 42 \%\right.$ yield). ${ }^{1} \mathrm{H}-\mathrm{NMR}\left(500 \mathrm{MHz}, \mathrm{CDCl}_{3}, \delta\right): 7.40-7.30(5 \mathrm{H}, \mathrm{Ar}-\underline{H})$, 5.21-5.06 (37H, Ar- $\underline{H}_{2} \mathrm{O}$ and $\left.\mathrm{OC} \underline{H}\left(\mathrm{CH}_{2}\right) \mathrm{CO}\right), 4.30\left(1 \mathrm{H},\left(\mathrm{CH}_{3}\right)_{3} \mathrm{Si}\left(\mathrm{CH}_{3}\right)_{2} \mathrm{OC} \underline{H}\left(\mathrm{CH}_{2}\right) \mathrm{CO}\right), 4.10-4.00(24 \mathrm{H}$, $\left.\mathrm{OCH}_{2} \mathrm{CH}_{2} \mathrm{CH}_{2}\right), 2.53-2.34\left(24 \mathrm{H}, \mathrm{CHCH}_{2} \mathrm{CH}_{2} \mathrm{CO}\right), 2.32-2.08\left(24 \mathrm{H}, \mathrm{CHCH}_{2} \mathrm{CH}_{2} \mathrm{CO}\right), 1.60-1.43$ (48H, $\mathrm{OCH}\left(\mathrm{CH}_{3}\right) \mathrm{CO}$ and $\left.\mathrm{OCH}_{2} \mathrm{CH}_{2} \mathrm{CH}_{2} \mathrm{CH}_{2}\right), \quad 1.39-1.15 \quad\left(216 \mathrm{H}, \quad \mathrm{OCH}_{2} \mathrm{CH}_{2}\left(\mathrm{C}_{2}\right)_{9} \mathrm{CH}_{3}\right) ; \quad 0.94-0.82 \quad(69 \mathrm{H}$, $\mathrm{CH}_{2} \mathrm{CH}_{2} \mathrm{C}_{3}$ and $\left.\mathrm{Si}\left(\mathrm{CH}_{3}\right)_{2}\left(\mathrm{C}_{3}\right)_{3}\right) ; 0.02\left(6 \mathrm{H}, \mathrm{Si}\left(\mathrm{C}_{3}\right)_{2}\left(\mathrm{CH}_{3}\right)_{3}\right)$. MS (MALDI-ToF): [M+Na] $]^{+} \mathrm{Cal} 5552.22 ; \mathrm{Obs}$ 5552.28 .

$\mathrm{D}_{14}-\mathrm{L}_{m}$ were obtained following the same protocol as $\mathrm{D}_{12}-\mathrm{L}_{m}$.

\subsection{2 $\mathrm{H}_{4} \mathrm{D}_{4} \mathrm{O}_{4}-\mathrm{L}_{m}$ and $\mathrm{H}_{4} \mathrm{D}_{6} \mathrm{O}_{4}-\mathrm{L}_{m}$}

Synthesis of $\mathrm{H}_{4} \underline{\mathrm{D}}_{4} \underline{\mathrm{O}}_{4}-\mathrm{COOH} . \mathrm{H}_{4} \mathrm{D}_{4} \mathrm{O}_{4}(500 \mathrm{mg})$ was dissolved in ethyl acetate (20 mL). Palladium (30 mg, $10 \%$ on carbon) was then added. The mixture was stirred at room temperature under hydrogen atmosphere for $6 \mathrm{~h}$. The black suspension was filtered through a thick layer of celite. After removal of solvent, the product $\left(\mathrm{H}_{4} \mathrm{D}_{4} \mathrm{O}_{4}-\mathrm{COOH}\right)$ was obtained in high purity (above $\left.95 \%\right)$ and used directly.

General Procedure for Synthesis of $\mathrm{H}_{4} \underline{\mathrm{D}}_{4} \underline{\mathrm{O}}_{4}-\mathrm{L}_{\underline{m}} . \mathrm{HO}-\mathrm{L}_{24}(40.0 \mathrm{mg}), \mathrm{H}_{4} \mathrm{D}_{4} \mathrm{O}_{4}-\mathrm{COOH}(80.0 \mathrm{mg})$, and DPTS $(100.0 \mathrm{mg})$ were mixed together into a round bottom flask. After transferring into a glove box, the mixture was dissolved in dry DCM. DIC $(0.50 \mathrm{~mL})$ was then added slowly at $0{ }^{\circ} \mathrm{C}$. The mixture was stirred at room temperature overnight. The solvent was removed, and the obtained crude product was purified by recycling preparative SEC, giving pure product $\mathrm{H}_{4} \mathrm{D}_{4} \mathrm{O}_{4}-\mathrm{L}_{24}$ (ca $50 \mathrm{mg}$, ca $42 \%$ yield). ${ }^{1} \mathrm{H}-\mathrm{NMR}\left(500 \mathrm{MHz}, \mathrm{CDCl}_{3}, \delta\right.$ ):

7.40-7.30 (5H, Ar- $\underline{H}), \quad 5.21-5.06 \quad\left(37 \mathrm{H}, \quad \operatorname{Ar}-\underline{C}_{2} \mathrm{O} \quad\right.$ and $\left.\quad \mathrm{OC} \underline{H}\left(\mathrm{CH}_{2}\right) \mathrm{CO}\right), \quad 4.30 \quad(1 \mathrm{H}$, $\left.\left(\mathrm{CH}_{3}\right)_{3} \mathrm{Si}\left(\mathrm{CH}_{3}\right)_{2} \mathrm{OC} \underline{H}\left(\mathrm{CH}_{2}\right) \mathrm{CO}\right), 4.10-4.00\left(24 \mathrm{H}, \mathrm{OC}_{2} \mathrm{CH}_{2} \mathrm{CH}_{2}\right), 2.53-2.34\left(24 \mathrm{H}, \mathrm{CHCH}_{2} \underline{\mathrm{H}}_{2} \mathrm{CO}\right), 2.32-2.08$ $\left(24 \mathrm{H}, \mathrm{CHC} \underline{H}_{2} \mathrm{CH}_{2} \mathrm{CO}\right), \quad 1.60-1.43\left(48 \mathrm{H}, \mathrm{OCH}\left(\underline{\mathrm{C}}_{3}\right) \mathrm{CO}\right.$ and $\left.\mathrm{OCH}_{2} \underline{\mathrm{H}}_{2} \mathrm{CH}_{2} \mathrm{CH}_{2}\right), \quad 1.39-1.15 \quad(120 \mathrm{H}$, $\mathrm{OCH}_{2} \mathrm{CH}_{2}\left(\mathrm{CH}_{2}\right)_{5} \mathrm{CH}_{3}, \mathrm{OCH}_{2} \mathrm{CH}_{2}\left(\mathrm{CH}_{2}\right)_{9} \mathrm{CH}_{3}$, and $\left.\mathrm{OCH}_{2} \mathrm{CH}_{2}\left(\mathrm{CH}_{2}\right)_{13} \mathrm{CH}_{3}\right), 0.94-0.82\left(45 \mathrm{H}, \mathrm{CH}_{2} \mathrm{CH}_{2} \mathrm{CH}_{3}\right.$ and $\left.\mathrm{Si}\left(\mathrm{CH}_{3}\right)_{2}\left(\mathrm{C}_{3}\right)_{3}\right), 0.02\left(6 \mathrm{H}, \mathrm{Si}\left(\mathrm{C}_{3}\right)_{2}\left(\mathrm{CH}_{3}\right)_{3}\right) . \mathrm{MS}$ (MALDI-ToF): [M+Na] $]^{+} \mathrm{Cal}$ 5552.22; Obs 5552.24.

$\mathrm{H}_{4} \mathrm{D}_{6} \mathrm{O}_{4}-\mathrm{L}_{m}$ were obtained following the same protocol as $\mathrm{H}_{4} \mathrm{D}_{4} \mathrm{O}_{4}-\mathrm{L}_{m}$. 


\subsection{3 $\mathrm{O}_{4} \mathrm{D}_{4} \mathrm{H}_{4}-\mathrm{L}_{\mathrm{m}}$ and $\mathrm{O}_{4} \mathrm{D}_{6} \mathrm{H}_{4}-\mathrm{L}_{\mathrm{m}}$}

Synthesis of $\mathrm{O}_{4} \underline{\mathrm{D}}_{4} \underline{\mathrm{H}}_{4}-\mathrm{COOH} . \mathrm{O}_{4} \mathrm{D}_{4} \mathrm{H}_{4}(500 \mathrm{mg})$ was dissolved in ethyl acetate (20 mL). Palladium (30 mg, $10 \%$ on carbon) was then added. The mixture was stirred at room temperature under a hydrogen atmosphere for 6 h. The black suspension was filtered through a thick layer of celite. After removal of solvent, the product $\left(\mathrm{O}_{4} \mathrm{D}_{4} \mathrm{H}_{4}-\mathrm{COOH}\right)$ was obtained in high purity (above $\left.95 \%\right)$ and used directly.

General Procedure for Synthesis of $\mathrm{O}_{4} \underline{\mathrm{D}}_{4} \underline{\mathrm{H}}_{4} \underline{\mathrm{L}}_{\underline{m}} . \mathrm{HO}-\mathrm{L}_{24}(40.0 \mathrm{mg}), \mathrm{O}_{4} \mathrm{D}_{4} \mathrm{H}_{4}-\mathrm{COOH}$ (80.0 mg), and DPTS $(100.0 \mathrm{mg})$ were mixed together into a round bottom flask. After transferring into a glove box, the mixture was dissolved in dry DCM. DIC $(0.50 \mathrm{~mL})$ was then added slowly at $0{ }^{\circ} \mathrm{C}$. The mixture was stirred at room temperature overnight. The solvent was removed, and the obtained crude product was purified by recycling preparative SEC, giving pure product $\mathrm{O}_{4} \mathrm{D}_{4} \mathrm{H}_{4}-\mathrm{L}_{24}\left(50 \mathrm{mg}, 42 \%\right.$ yield). ${ }^{1} \mathrm{H}-\mathrm{NMR}\left(500 \mathrm{MHz}, \mathrm{CDCl}_{3}, \delta\right): 7.40-$ $7.30(5 \mathrm{H}, \mathrm{Ar}-\underline{H}), 5.21-5.06\left(37 \mathrm{H}, \mathrm{Ar}-\mathrm{C}_{2} \mathrm{O}\right.$ and $\left.\mathrm{OC} \underline{H}\left(\mathrm{CH}_{2}\right) \mathrm{CO}\right), 4.30\left(1 \mathrm{H},\left(\mathrm{CH}_{3}\right)_{3} \mathrm{Si}\left(\mathrm{CH}_{3}\right)_{2} \mathrm{OC} \underline{H}\left(\mathrm{CH}_{2}\right) \mathrm{CO}\right)$, 4.10-4.00 (24H, OC $\left.\underline{H}_{2} \mathrm{CH}_{2} \mathrm{CH}_{2}\right), 2.53-2.34\left(24 \mathrm{H}, \mathrm{CHCH}_{2} \mathrm{CH}_{2} \mathrm{CO}\right), 2.32-2.08\left(24 \mathrm{H}, \mathrm{CHCH}_{2} \mathrm{CH}_{2} \mathrm{CO}\right), 1.60-$ $1.43\left(48 \mathrm{H}, \quad \mathrm{OCH}\left(\mathrm{CH}_{3}\right) \mathrm{CO} \quad\right.$ and $\left.\quad \mathrm{OCH}_{2} \mathrm{CH}_{2} \mathrm{CH}_{2} \mathrm{CH}_{2}\right), \quad 1.39-1.15 \quad\left(120 \mathrm{H}, \quad \mathrm{OCH}_{2} \mathrm{CH}_{2}\left(\mathrm{CH}_{2}\right)_{5} \mathrm{CH}_{3}\right.$, $\mathrm{OCH}_{2} \mathrm{CH}_{2}\left(\mathrm{C}_{2}\right)_{9} \mathrm{CH}_{3}$, and $\left.\mathrm{OCH}_{2} \mathrm{CH}_{2}\left(\mathrm{C}_{2}\right)_{13} \mathrm{CH}_{3}\right), 0.94-0.82\left(45 \mathrm{H}, \mathrm{CH}_{2} \mathrm{CH}_{2} \mathrm{C}_{3}\right.$ and $\left.\mathrm{Si}\left(\mathrm{CH}_{3}\right)_{2}\left(\mathrm{C}_{3}\right)_{3}\right), 0.02$ $\left(6 \mathrm{H}, \mathrm{Si}\left(\mathrm{CH}_{3}\right)_{2}\left(\mathrm{CH}_{3}\right)_{3}\right) . \mathrm{MS}(\mathrm{MALDI}-\mathrm{ToF}):[\mathrm{M}+\mathrm{Na}]^{+} \mathrm{Cal}$ 5552.22; Obs 5552.21.

$\mathrm{O}_{4} \mathrm{D}_{6} \mathrm{H}_{4}-\mathrm{L}_{m}$ were obtained following the same protocol as $\mathrm{O}_{4} \mathrm{D}_{4} \mathrm{H}_{4}-\mathrm{L}_{m}$.

\section{Supplementary Calculation and Discussion}

\subsection{Measure of Density and Calculation of Volume Fraction}

Densities of discrete polymer were measured by pipetting $100 \mu \mathrm{L}$ of viscous liquids using highly precise pipette and weighing the mass. Each measurement was repeated three times. Density of $\mathrm{B}_{n}, \mathrm{O}_{n}, \mathrm{D}_{n}$, and $\mathrm{H}_{n}$ was determined as $1.172,1.076,1.041$, and $1.008 \mathrm{~g} / \mathrm{cm}^{3}$, respectively.

The volume fraction of $o \mathrm{LA}\left(f_{\mathrm{LA}}\right)$ in diblock copolymers is calculated by Eq $(\mathrm{S} 1)$

$$
f_{L A}=\frac{M_{L} / \rho_{L}}{M_{L} / \rho_{L}+\sum\left(M_{C_{i}} / \rho_{C_{i}}\right)}
$$

where $M_{\mathrm{L}}$ and $M_{\mathrm{C}}$ are the molecular weight of $o$ LA and $o$ CGA fragments; $\rho_{\mathrm{L}}$ and $\rho_{\mathrm{C}}$ are the densities, respectively. According to literature, $\rho_{\mathrm{L}}=1.25 \mathrm{~g} / \mathrm{cm}^{3}$.

\subsection{Characteristic dimension of the phase $(a)$}


The dimension of the phase ( $a$ ) refers to inter-column distances (for HEX), or lattice parameters (for A15 and $\sigma)$. It can be calculated from corresponding diffraction peaks accordingly.

$$
\begin{array}{ll}
a=2 / \sqrt{3} d_{10} & \text { for HEX } \\
a=\sqrt{2} d_{110} & \text { for A15 } \\
a=\sqrt{17} d_{410} ; c=2 d_{002} & \text { for } \sigma
\end{array}
$$

3.3 Average diameter of spherical motifs of A15 and $\sigma$ phases $(D)$

$$
\begin{array}{lr}
\langle D\rangle=\sqrt[3]{\frac{3}{4 \pi}} a & \text { for A15 } \\
\langle D\rangle=\sqrt[3]{\frac{V}{5 \pi}} & \text { for } \sigma
\end{array}
$$

where $a$ refers to the cubic lattice parameter of A15, and $V$ stands for the lattice volume of $\sigma$ phase $\left(V=a^{*} a^{*} c\right)$.

3.4 Number of molecules within one spherical motif $(\mu)$

$$
\begin{array}{lr}
\mu=\frac{\rho a^{3}}{8 m} \times N_{A} & \text { for A15 } \\
\mu=\frac{\rho V}{30 m} \times N_{A} & \text { for } \sigma
\end{array}
$$

where $a$ is the cubic lattice parameter of A15, $V$ is the lattice volume of $\sigma$ phase $\left(V=a^{*} a^{*} c\right), m$ is the molecular weight, and $N_{\mathrm{A}}$ is Avogadro's number.

For a HEX phase, the average number of molecules $\mu$ is calculated by:

$$
\mu=\frac{\rho V}{m} \times N_{A}=\frac{2 \rho d_{1}^{2} h}{\sqrt{3} m} \times N_{A}
$$

where $d_{1}$ is the distance between (10) plane, $h$ is the thickness of cylinder, $m$ is the molecular weight, and $N_{\mathrm{A}}$ is Avogadro's number. Note that the cylinders are not discrete and therefore the aggregation number depends on the thickness $(h)$. For convenience, we take $h=1.0 \mathrm{~nm}$, and assume block copolymers are uniformly arranged within the slice. The results are relative values. 


$$
\begin{array}{cr}
\theta=\frac{2 \pi}{\mu} & \text { for HEX } \\
\theta=2 \arccos \left(1-\frac{2}{\mu}\right) & \text { for A15 and } \sigma
\end{array}
$$

\subsection{Asymmetric parameter $(\alpha)$ of $\mathrm{H}_{n 1} \mathrm{D}_{n 2} \mathrm{O}_{n 3}$ and $\mathrm{O}_{n 1} \mathrm{D}_{n 2} \mathrm{H}_{n 3}$}

Total number of methylene/methyl units on the side chain for $\mathrm{D}_{12}$ and $\mathrm{D}_{14}$ series: $X_{0,12}=12 * 12=144 ; X_{0,14}=$ $14 * 12=168$

\begin{tabular}{cccc}
\hline Sample & $X_{r}$ & $X_{0}$ & $\alpha$ \\
\hline $\mathrm{H}_{4} \mathrm{D}_{4} \mathrm{O}_{4}$ & 56 & 144 & -0.22 \\
$\mathrm{D}_{12}$ & 72 & 144 & 0 \\
$\mathrm{O}_{4} \mathrm{D}_{4} \mathrm{H}_{4}$ & 88 & 144 & 0.22 \\
$\mathrm{H}_{4} \mathrm{D}_{6} \mathrm{O}_{4}$ & 68 & 168 & -0.19 \\
$\mathrm{D}_{14}$ & 84 & 168 & 0 \\
$\mathrm{O}_{4} \mathrm{D}_{6} \mathrm{H}_{4}$ & 100 & 168 & 0.19 \\
\hline
\end{tabular}

\begin{tabular}{|c|c|c|c|c|c|c|c|}
\hline Peak NO. & (hkl) & $q_{\mathrm{cal}}\left(\mathrm{nm}^{-1}\right)$ & $q_{\text {obs }}\left(\mathrm{nm}^{-1}\right)$ & Peak NO. & (hkl) & $q_{\mathrm{cal}}\left(\mathrm{nm}^{-1}\right)$ & $q_{\mathrm{obs}}\left(\mathrm{nm}^{-1}\right)$ \\
\hline 1 & 110 & 0.239 & & 21 & 420 & 0.757 & \\
\hline 2 & 200 & 0.339 & & 22 & 411 & 0.768 & 0.767 \\
\hline 3 & 101 & 0.362 & & 23 & 331 & 0.786 & \\
\hline 4 & 210 & 0.379 & & 24 & 222 & 0.799 & \\
\hline 5 & 111 & 0.400 & & 25 & 421 & 0.822 & \\
\hline 6 & 220 & 0.479 & & 26 & 312 & 0.835 & \\
\hline 7 & 211 & 0.496 & & 27 & 430 & 0.847 & \\
\hline 8 & 310 & 0.535 & 0.537 & 28 & 510 & 0.863 & \\
\hline 9 & 221 & 0.576 & 0.577 & 29 & 322 & 0.885 & 0.883 \\
\hline 10 & 301 & 0.600 & & 30 & 501 & 0.905 & \\
\hline 11 & 320 & 0.610 & & 31 & 431 & 0.905 & \\
\hline 12 & 311 & 0.624 & 0.625 & 32 & 520 & 0.912 & 0.915 \\
\hline 13 & 002 & 0.640 & 0.641 & 33 & 511 & 0.921 & \\
\hline 14 & 400 & 0.677 & & 34 & 402 & 0.932 & \\
\hline 15 & 112 & 0.683 & & 35 & 412 & 0.947 & \\
\hline 16 & 321 & 0.689 & & 36 & 440 & 0.958 & \\
\hline 17 & 410 & 0.698 & 0.698 & 37 & 332 & 0.962 & \\
\hline 18 & 330 & 0.718 & & 38 & 521 & 0.966 & \\
\hline
\end{tabular}

3.7 Index of peaks in the SAXS pattern of $D_{14}-L_{26}(\sigma$ phase $)$ 


\begin{tabular}{llll|lll}
$\mathbf{1 9}$ & 202 & 0.724 & 0.723 & $\mathbf{3 9}$ & 103 & 0.975 \\
$\mathbf{2 0}$ & 212 & 0.744 & 0.743 & & & \\
\hline
\end{tabular}

3.8 Index of peaks in the SAXS pattern of $\mathrm{O}_{4} \mathrm{D}_{4} \mathrm{H}_{6}-\mathrm{L}_{28}(\sigma$ phase)

\begin{tabular}{|c|c|c|c|c|c|c|c|}
\hline Peak NO. & (hkl) & $q_{\text {cal }}\left(\mathrm{nm}^{-1}\right)$ & $q_{\text {obs }}\left(\mathrm{nm}^{-1}\right)$ & Peak NO. & (hkl) & $q_{\text {cal }}\left(\mathrm{nm}^{-1}\right)$ & $q_{\mathrm{obs}}\left(\mathrm{nm}^{-1}\right)$ \\
\hline 1 & 110 & 0.243 & & 21 & 420 & 0.769 & \\
\hline 2 & 200 & 0.344 & & 22 & 411 & 0.781 & 0.780 \\
\hline 3 & 101 & 0.369 & & 23 & 331 & 0.800 & \\
\hline 4 & 210 & 0.385 & & 24 & 222 & 0.814 & \\
\hline 5 & 111 & 0.407 & & 25 & 421 & 0.836 & \\
\hline 6 & 220 & 0.487 & & 26 & 312 & 0.850 & 0.847 \\
\hline 7 & 211 & 0.505 & & 27 & 430 & 0.860 & \\
\hline 8 & 310 & 0.544 & 0.545 & 28 & 510 & 0.877 & \\
\hline 9 & 221 & 0.586 & 0.587 & 29 & 322 & 0.901 & 0.897 \\
\hline 10 & 301 & 0.611 & & 30 & 501 & 0.920 & \\
\hline 11 & 320 & 0.620 & & 31 & 431 & 0.920 & \\
\hline 12 & 311 & 0.634 & & 32 & 520 & 0.927 & \\
\hline 13 & 002 & 0.653 & 0.654 & 33 & 511 & 0.936 & \\
\hline 14 & 400 & 0.688 & & 34 & 402 & 0.949 & \\
\hline 15 & 112 & 0.697 & & 35 & 412 & 0.964 & \\
\hline 16 & 321 & 0.701 & & 36 & 440 & 0.973 & \\
\hline 17 & 410 & 0.709 & 0.710 & 37 & 332 & 0.979 & \\
\hline 18 & 330 & 0.730 & & 38 & 521 & 0.982 & \\
\hline 19 & 202 & 0.738 & 0.738 & 39 & 103 & 0.994 & \\
\hline 20 & 212 & 0.758 & 0.738 & & & & \\
\hline
\end{tabular}




\section{Supplementary Figures and Tables}

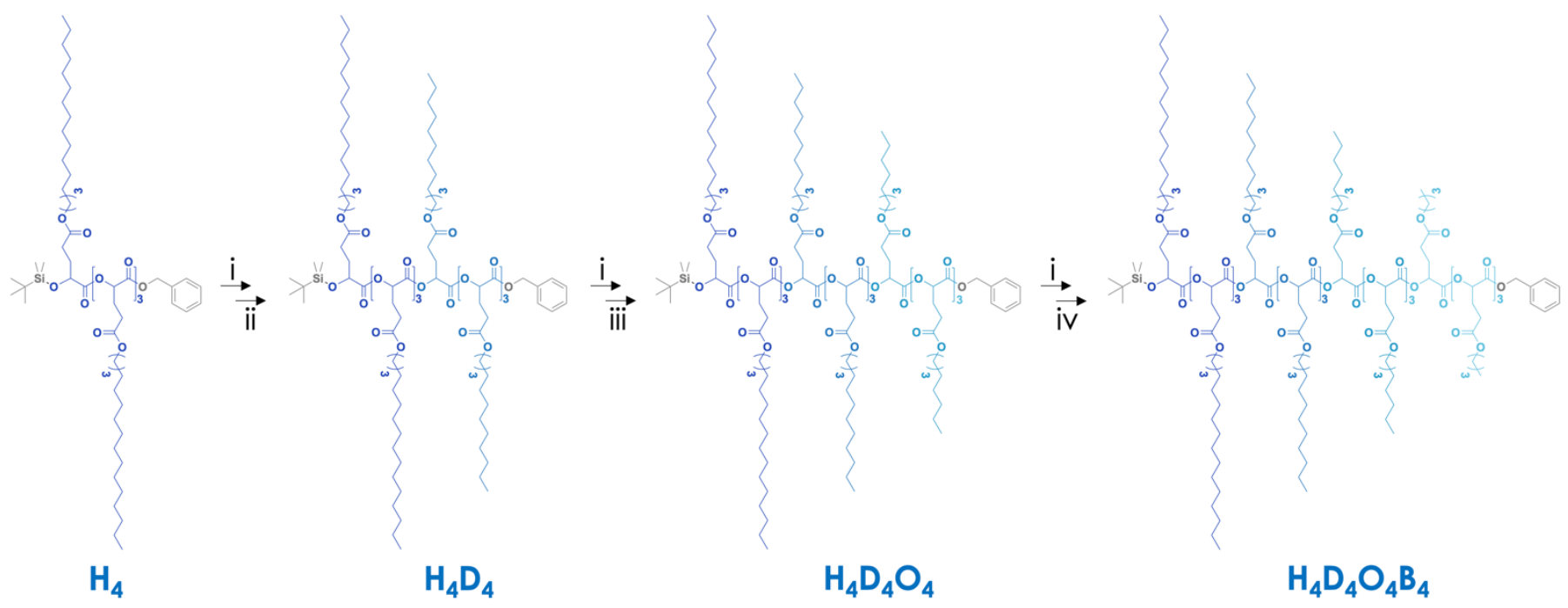

Scheme S1. Synthetic route of $\mathrm{H}_{4} \mathrm{D}_{4} \mathrm{O}_{4} \mathrm{~B}_{4}$.

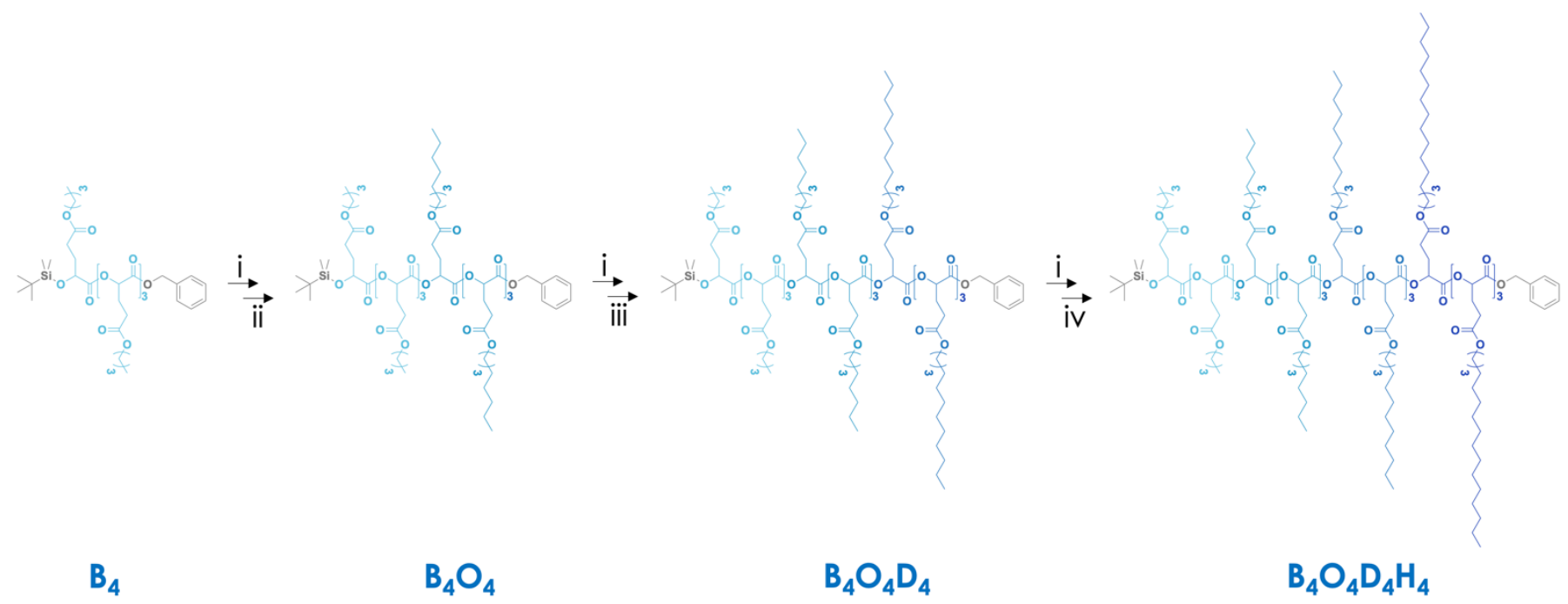

Scheme S2. Synthetic route of $\mathrm{B}_{4} \mathrm{O}_{4} \mathrm{D}_{4} \mathrm{H}_{4}$. 
(a)

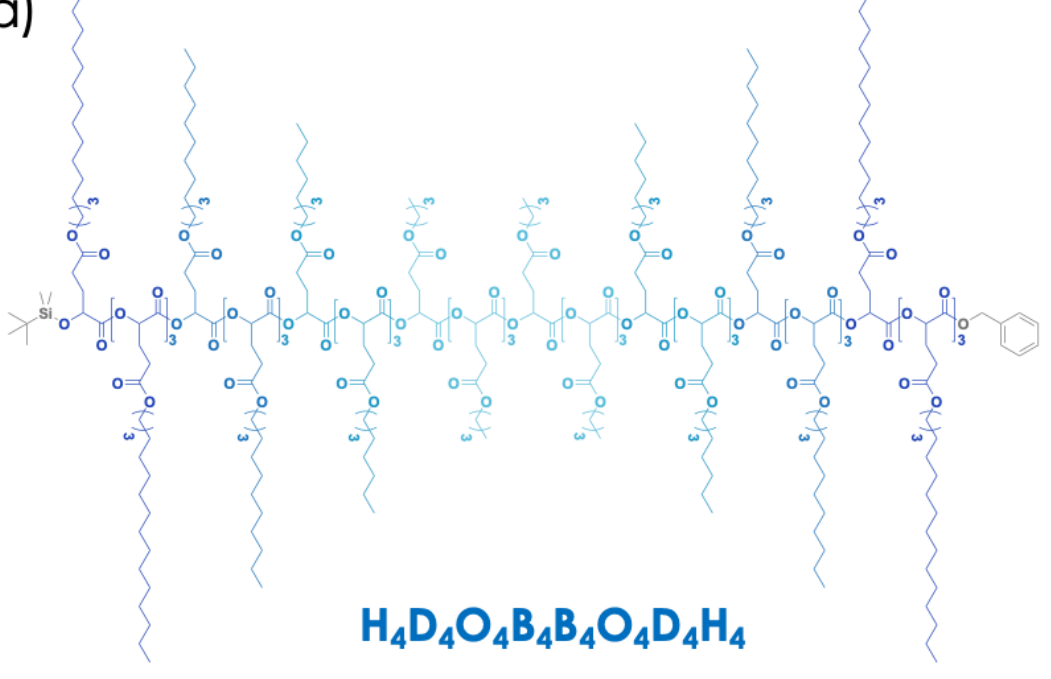

(b)

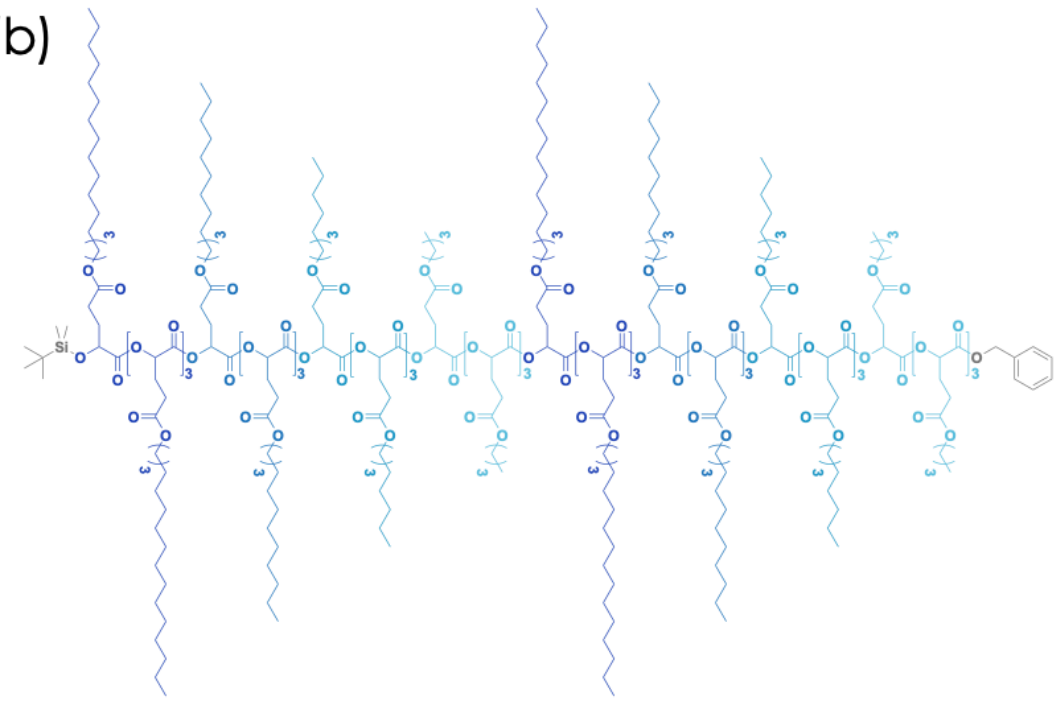

$\mathrm{H}_{4} \mathrm{D}_{4} \mathrm{O}_{4} \mathrm{~B}_{4} \mathrm{H}_{4} \mathrm{D}_{4} \mathrm{O}_{4} \mathrm{~B}_{4}$

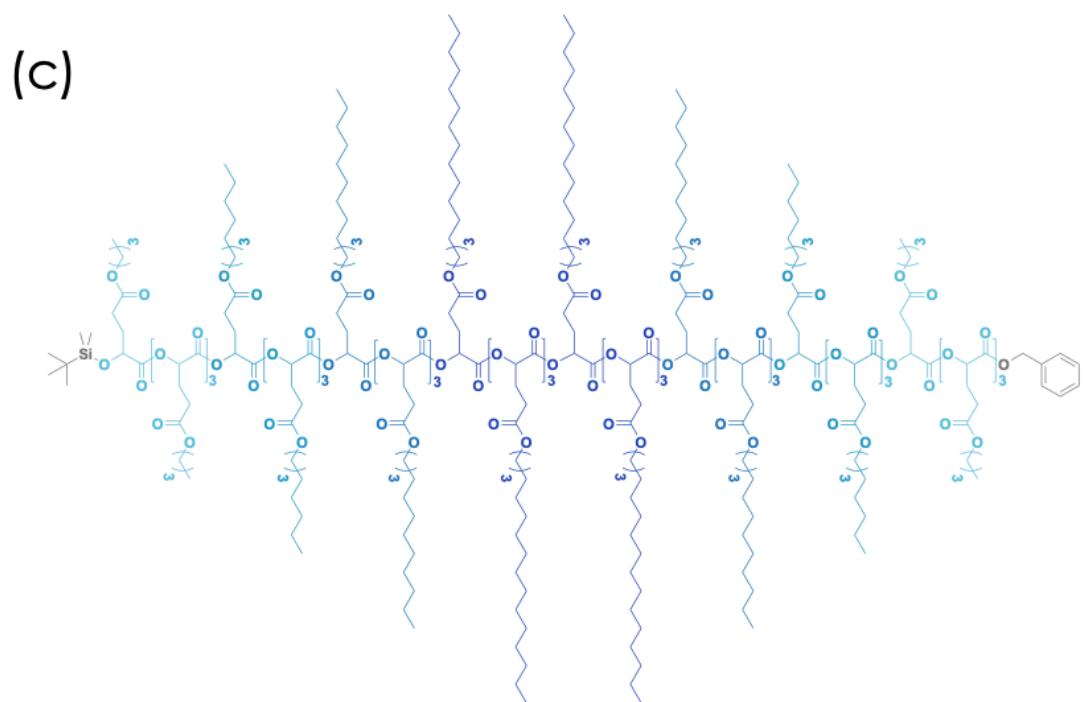

$\mathrm{B}_{4} \mathrm{O}_{4} \mathrm{D}_{4} \mathrm{H}_{4} \mathrm{H}_{4} \mathrm{D}_{4} \mathrm{O}_{4} \mathrm{~B}_{4}$

Scheme S3. Chemical Structures of $\mathrm{H}_{4} \mathrm{D}_{4} \mathrm{O}_{4} \mathrm{~B}_{4} \mathrm{~B}_{4} \mathrm{O}_{4} \mathrm{D}_{4} \mathrm{H}_{4}$ (a), $\mathrm{H}_{4} \mathrm{D}_{4} \mathrm{O}_{4} \mathrm{~B}_{4} \mathrm{H}_{4} \mathrm{D}_{4} \mathrm{O}_{4} \mathrm{~B}_{4}$ (b), and $\mathrm{B}_{4} \mathrm{O}_{4} \mathrm{D}_{4} \mathrm{H}_{4} \mathrm{H}_{4} \mathrm{D}_{4} \mathrm{O}_{4} \mathrm{~B}_{4}$ (c). 


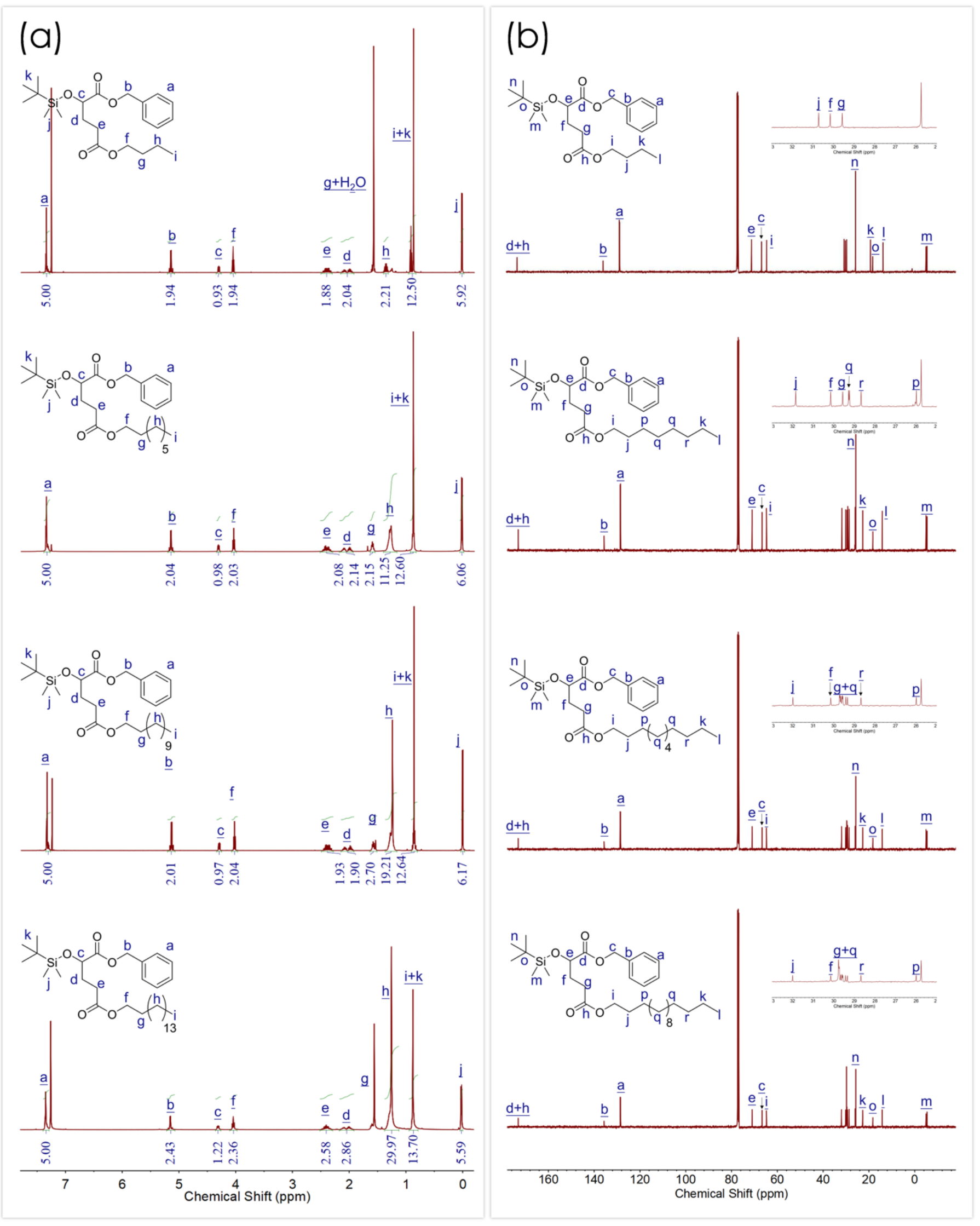

Figure S1. ${ }^{1} \mathrm{H}$ NMR (a) and ${ }^{13} \mathrm{C}$ NMR spectra (b) of CGA monomers. From top to bottom: BGA, OGA, DGA, and HGA. 

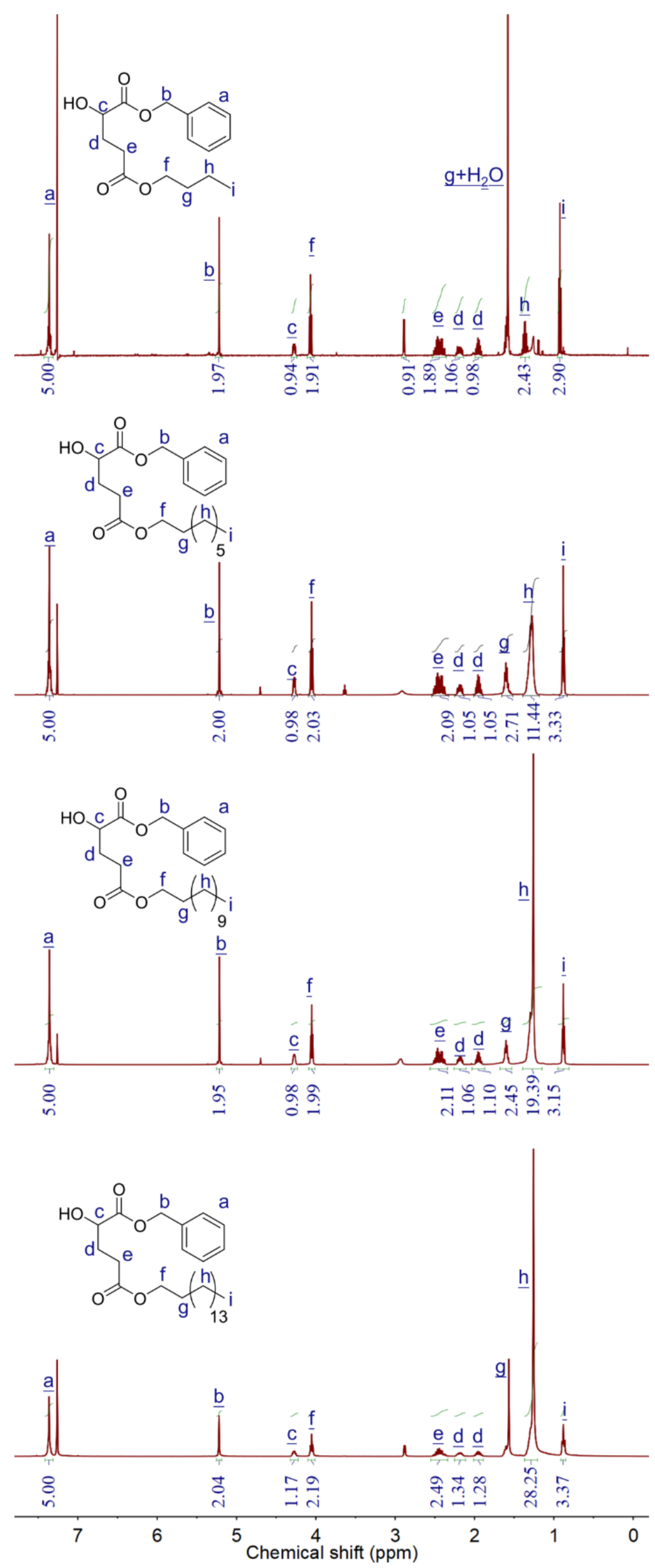

Figure S2. ${ }^{1} \mathrm{H}$ NMR spectra of deprotected monomers. From top to bottom: BGA-OH, OGA-OH, DGA-OH, and HGA-OH. 


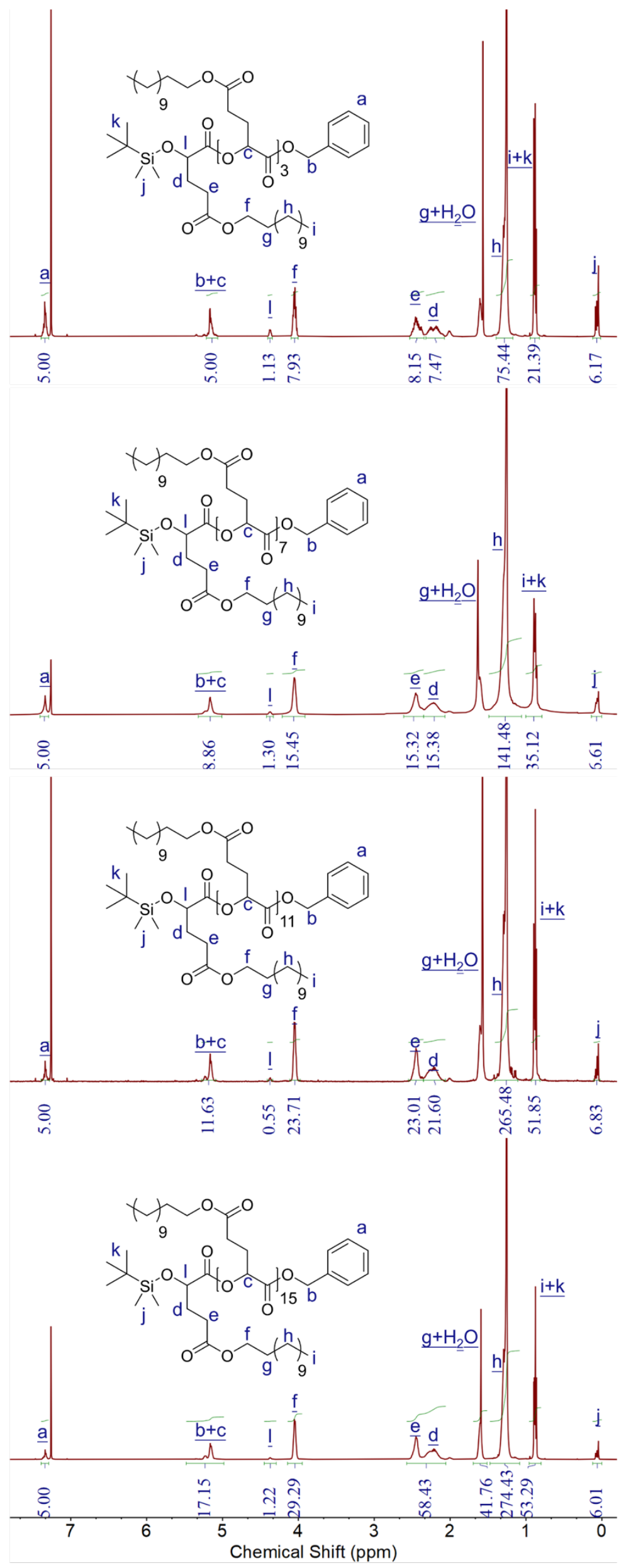

Figure S3. ${ }^{1} \mathrm{H}$ NMR spectra of discrete polymers $\mathrm{D}_{n}$. From top to bottom: $n=4,8,12$, and 16 . 

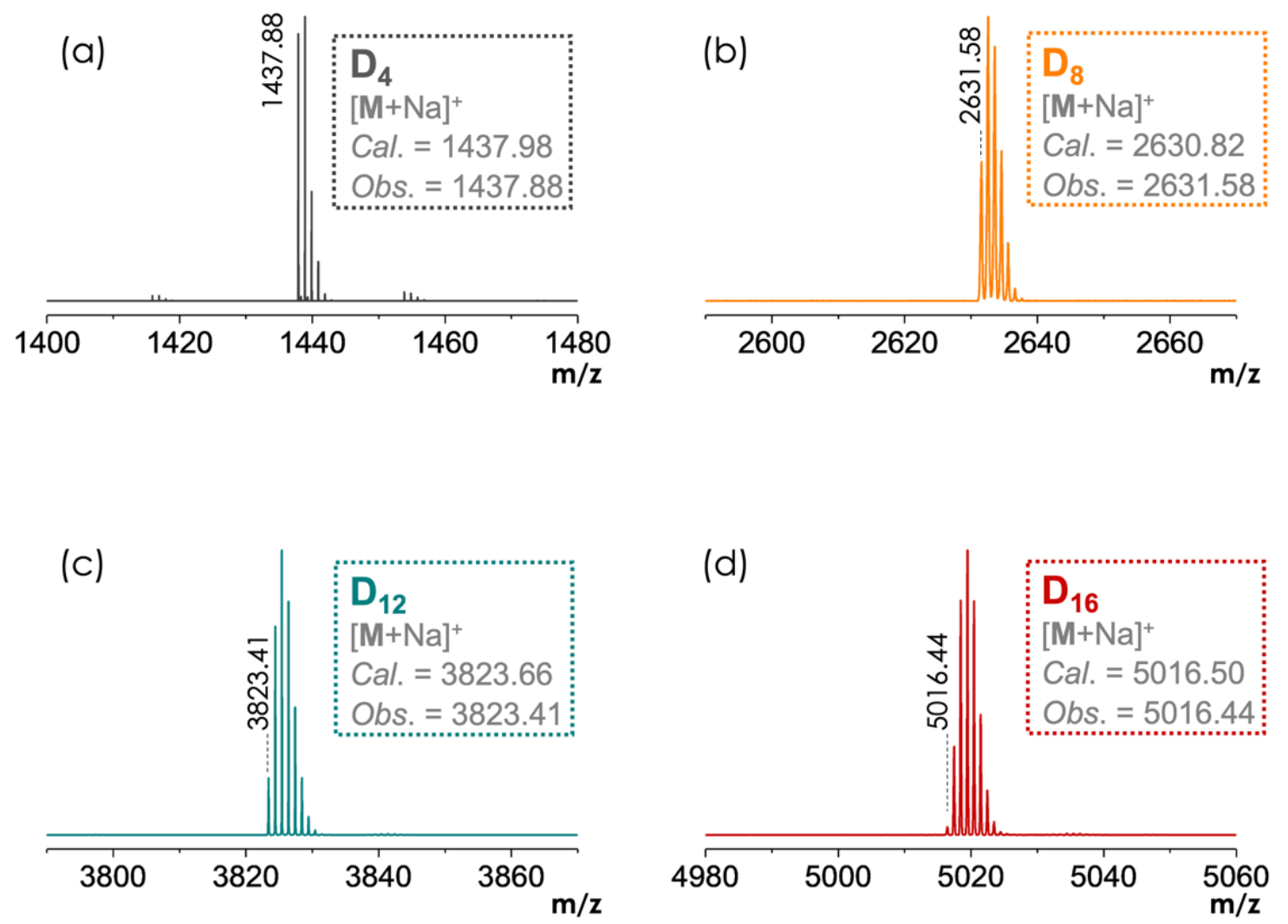

Figure S4. Enlarged MALDI-ToF MS of $\mathrm{D}_{4}(\mathrm{a}), \mathrm{D}_{8}(\mathrm{~b}), \mathrm{D}_{12}(\mathrm{c})$, and $\mathrm{D}_{16}(\mathrm{~d})$.

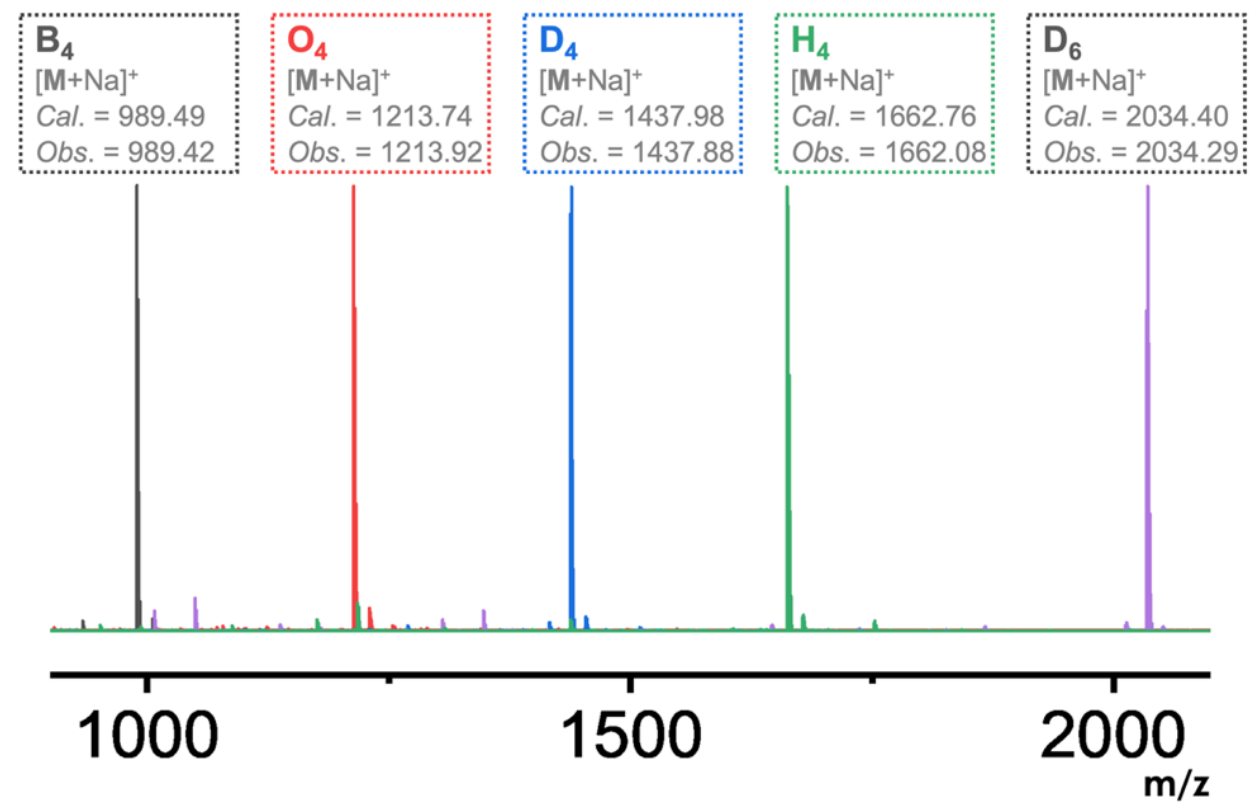

Figure S5. MALDI-ToF MS of fragments. From left to right: $\mathrm{B}_{4}, \mathrm{O}_{4}, \mathrm{D}_{4}, \mathrm{H}_{4}$, and $\mathrm{D}_{6}$. 


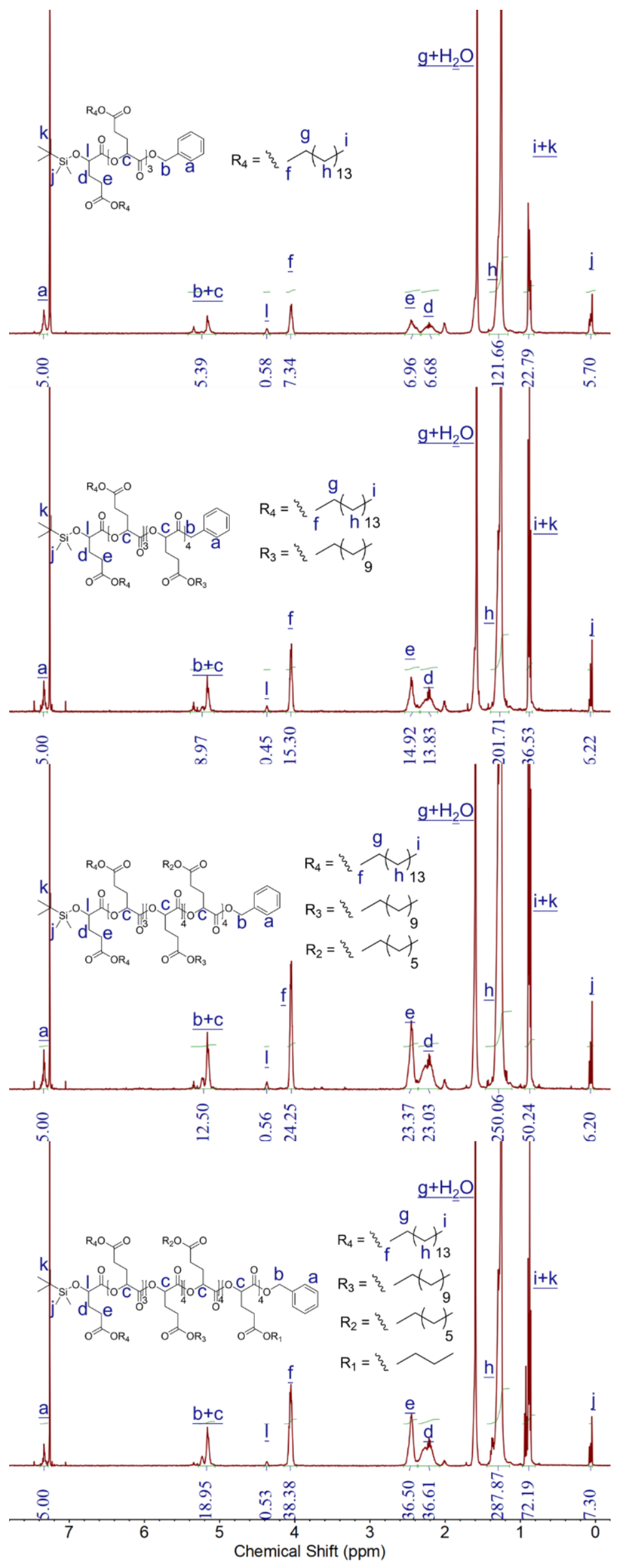

Figure S6. ${ }^{1} \mathrm{H}$ NMR spectra of $\mathrm{H}_{4}, \mathrm{H}_{4} \mathrm{D}_{4}, \mathrm{H}_{4} \mathrm{D}_{4} \mathrm{O}_{4}$, and $\mathrm{H}_{4} \mathrm{D}_{4} \mathrm{O}_{4} \mathrm{~B}_{4}$ (from top to bottom). 

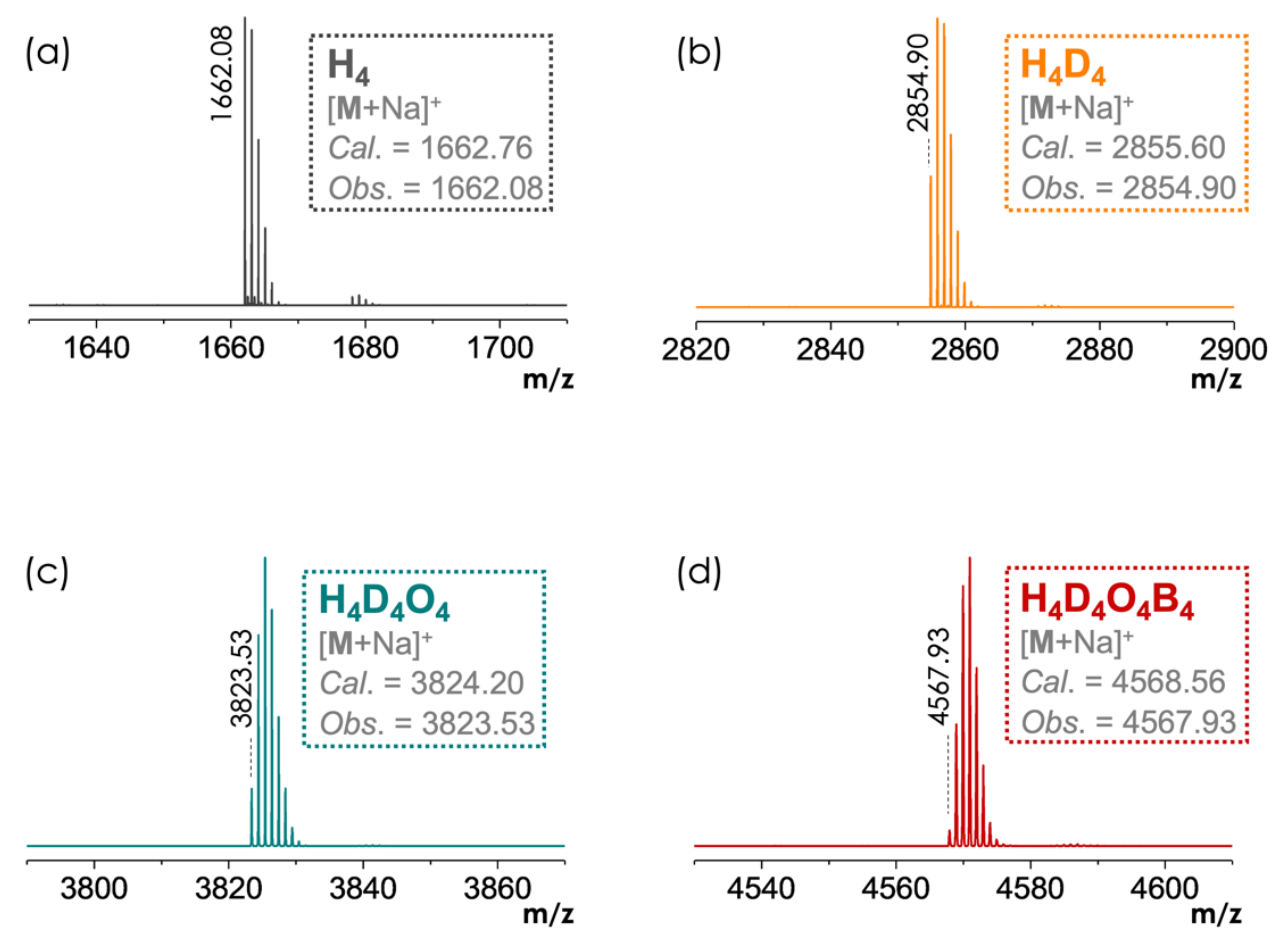

Figure S7. Enlarged MALDI-ToF MS of $\mathrm{H}_{4}(\mathrm{a}), \mathrm{H}_{4} \mathrm{D}_{4}(\mathrm{~b}), \mathrm{H}_{4} \mathrm{D}_{4} \mathrm{O}_{4}$ (c), and $\mathrm{H}_{4} \mathrm{D}_{4} \mathrm{O}_{4} \mathrm{~B}_{4}$ (d).
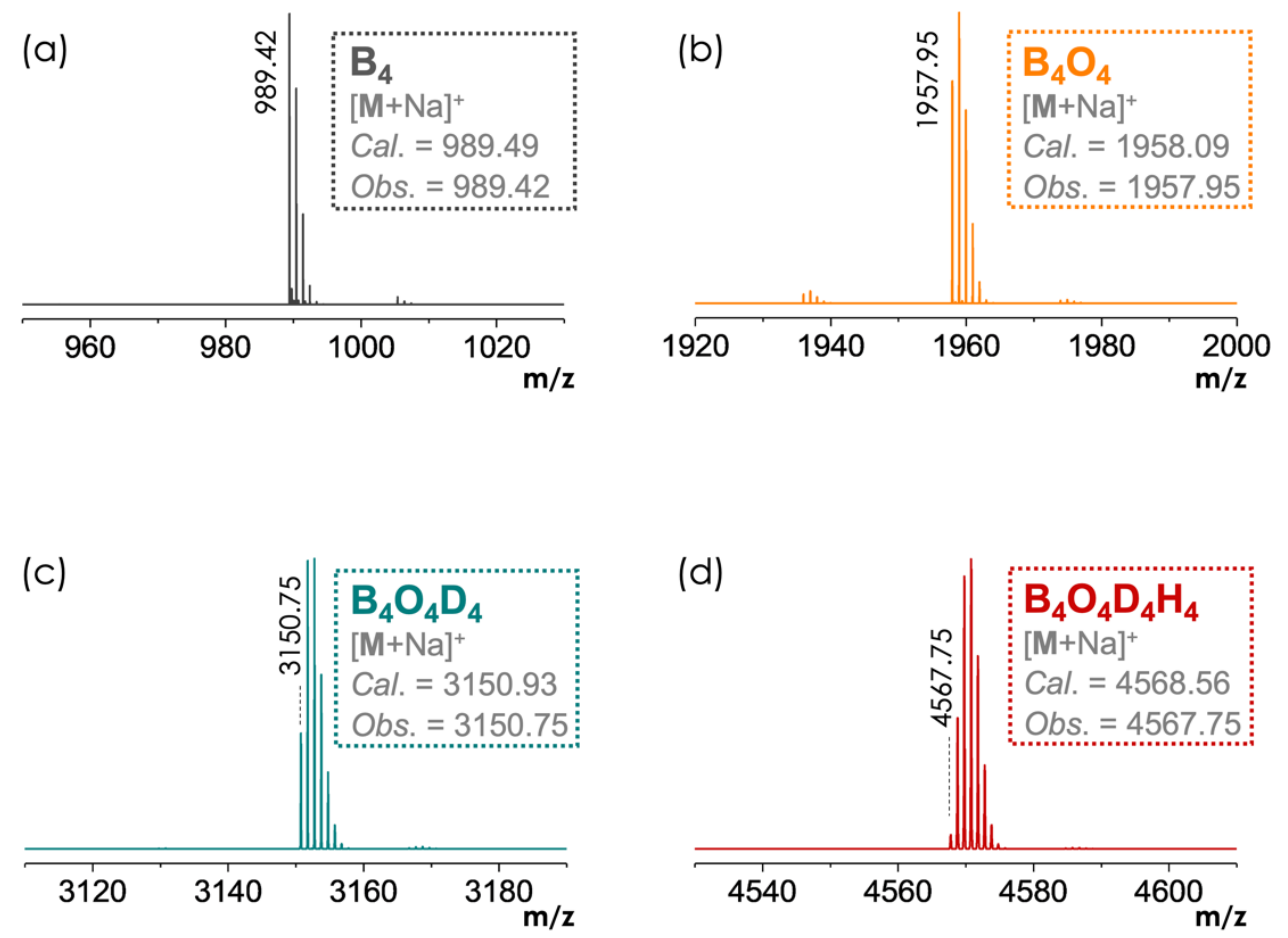

Figure S8. Enlarged MALDI-ToF MS of $\mathrm{B}_{4}(\mathrm{a}), \mathrm{B}_{4} \mathrm{O}_{4}(\mathrm{~b}), \mathrm{B}_{4} \mathrm{O}_{4} \mathrm{D}_{4}(\mathrm{c})$, and $\mathrm{B}_{4} \mathrm{O}_{4} \mathrm{D}_{4} \mathrm{H}_{4}(\mathrm{~d})$. 


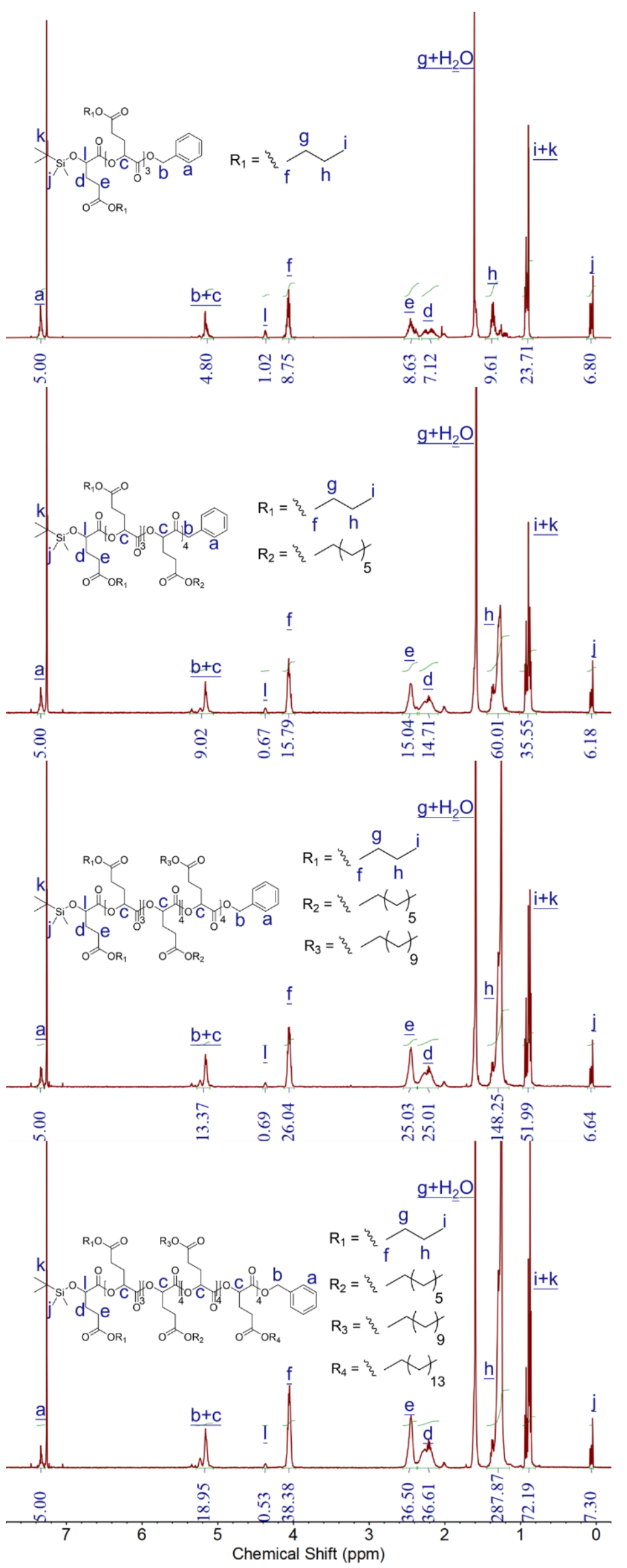

Figure S9. ${ }^{1} \mathrm{H}$ NMR spectra of $\mathrm{B}_{4}, \mathrm{~B}_{4} \mathrm{O}_{4}, \mathrm{~B}_{4} \mathrm{O}_{4} \mathrm{D}_{4}$, and $\mathrm{B}_{4} \mathrm{O}_{4} \mathrm{D}_{4} \mathrm{H}_{4}$ (from top to bottom). 
(a)

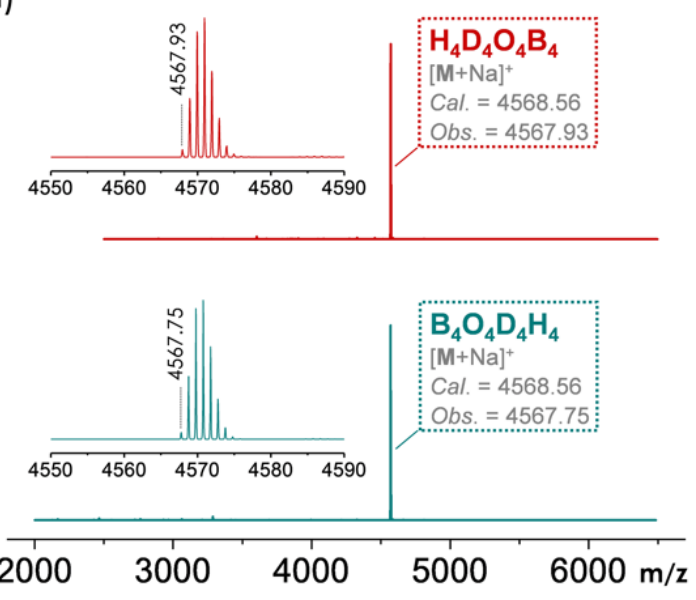

(b)

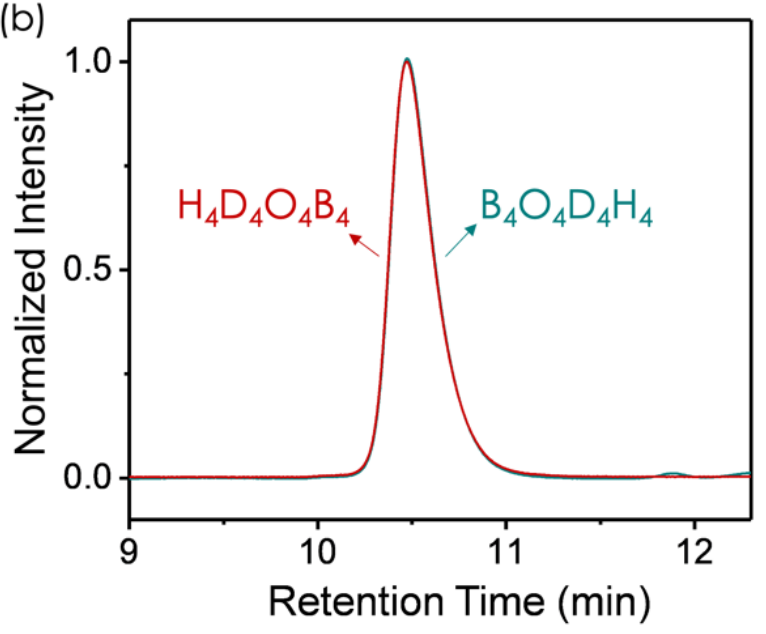

Figure S10. MALDI-ToF MS (a) and SEC traces (b) of $\mathrm{H}_{4} \mathrm{D}_{4} \mathrm{O}_{4} \mathrm{~B}_{4}$ and $\mathrm{B}_{4} \mathrm{O}_{4} \mathrm{D}_{4} \mathrm{H}_{4}$.

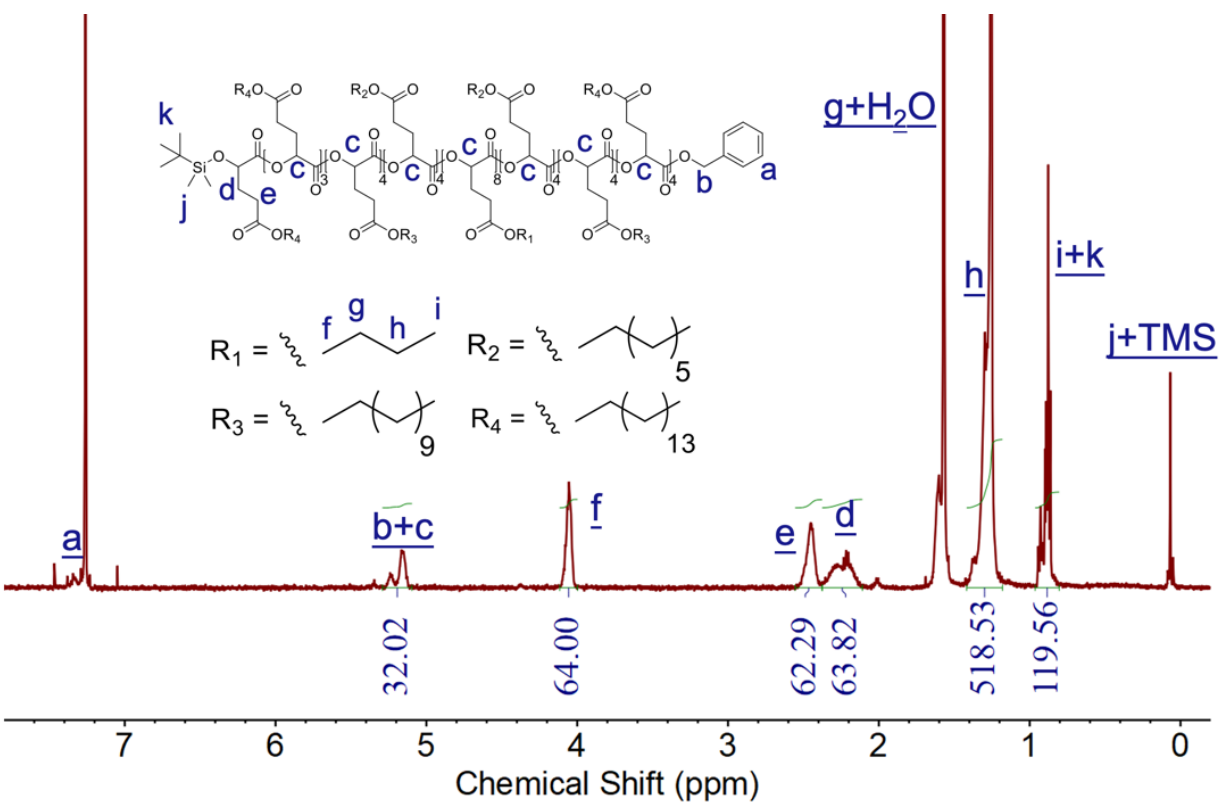

Figure S11. ${ }^{1} \mathrm{H}$ NMR of discrete polymer $\mathrm{H}_{4} \mathrm{D}_{4} \mathrm{O}_{4} \mathrm{~B}_{4} \mathrm{~B}_{4} \mathrm{O}_{4} \mathrm{D}_{4} \mathrm{H}_{4}$. 


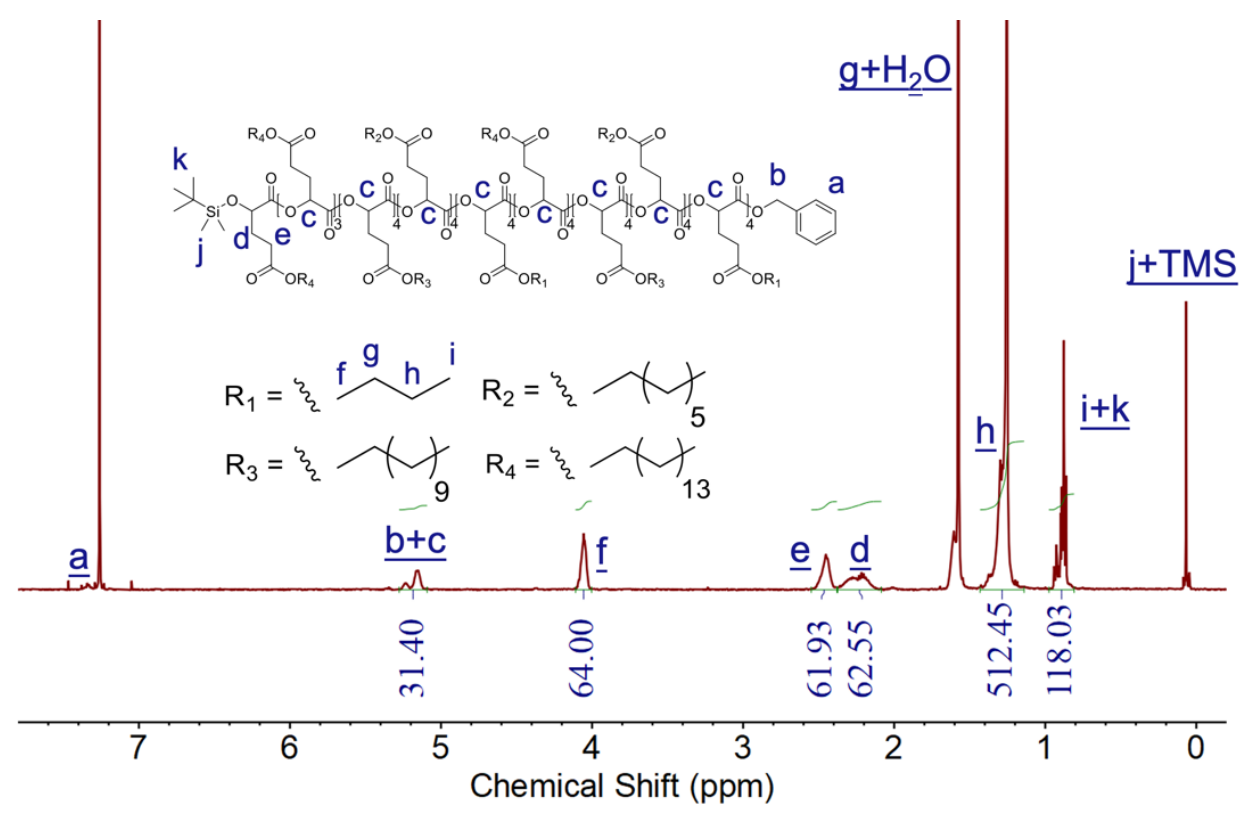

Figure S12. ${ }^{1} \mathrm{H}$ NMR of discrete polymer $\mathrm{H}_{4} \mathrm{D}_{4} \mathrm{O}_{4} \mathrm{~B}_{4} \mathrm{H}_{4} \mathrm{D}_{4} \mathrm{O}_{4} \mathrm{~B}_{4}$.

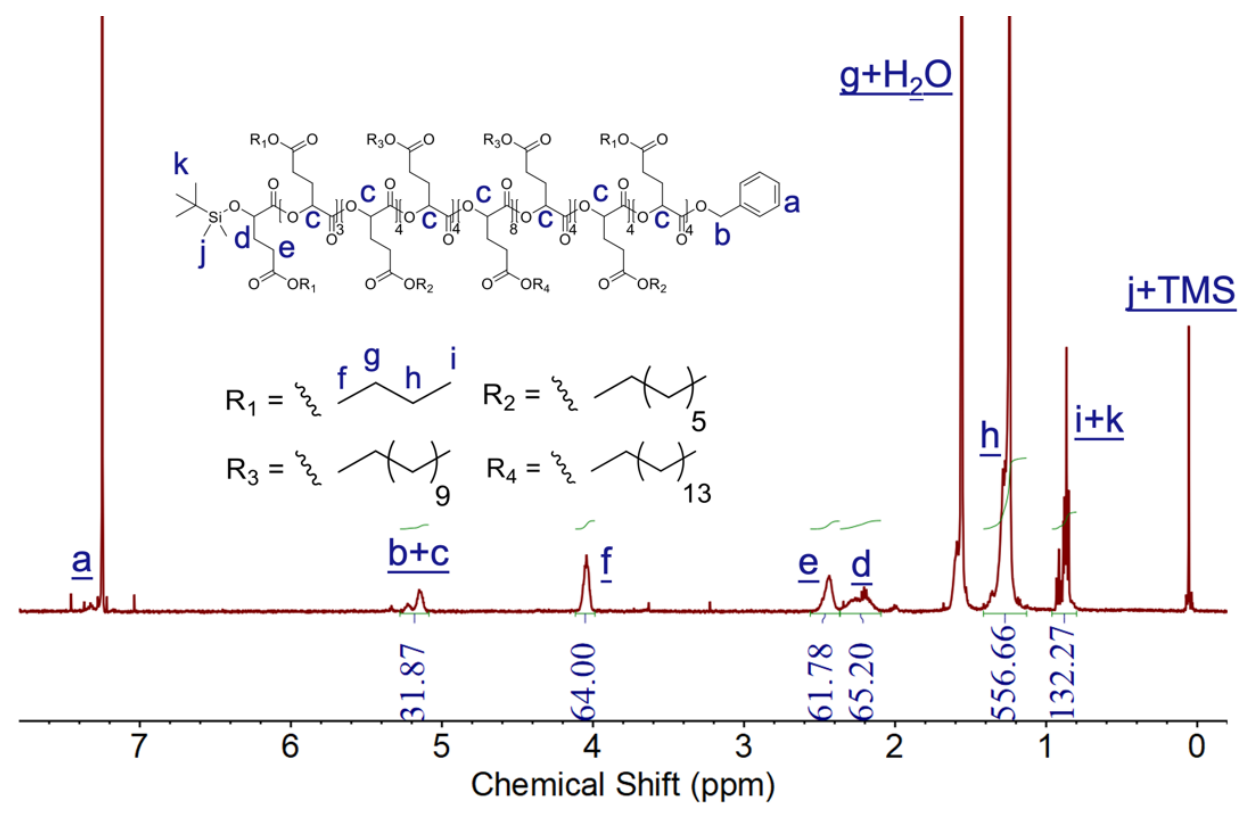

Figure S13. ${ }^{1} \mathrm{H}$ NMR of discrete polymer $\mathrm{B}_{4} \mathrm{O}_{4} \mathrm{D}_{4} \mathrm{H}_{4} \mathrm{H}_{4} \mathrm{D}_{4} \mathrm{O}_{4} \mathrm{~B}_{4}$. 


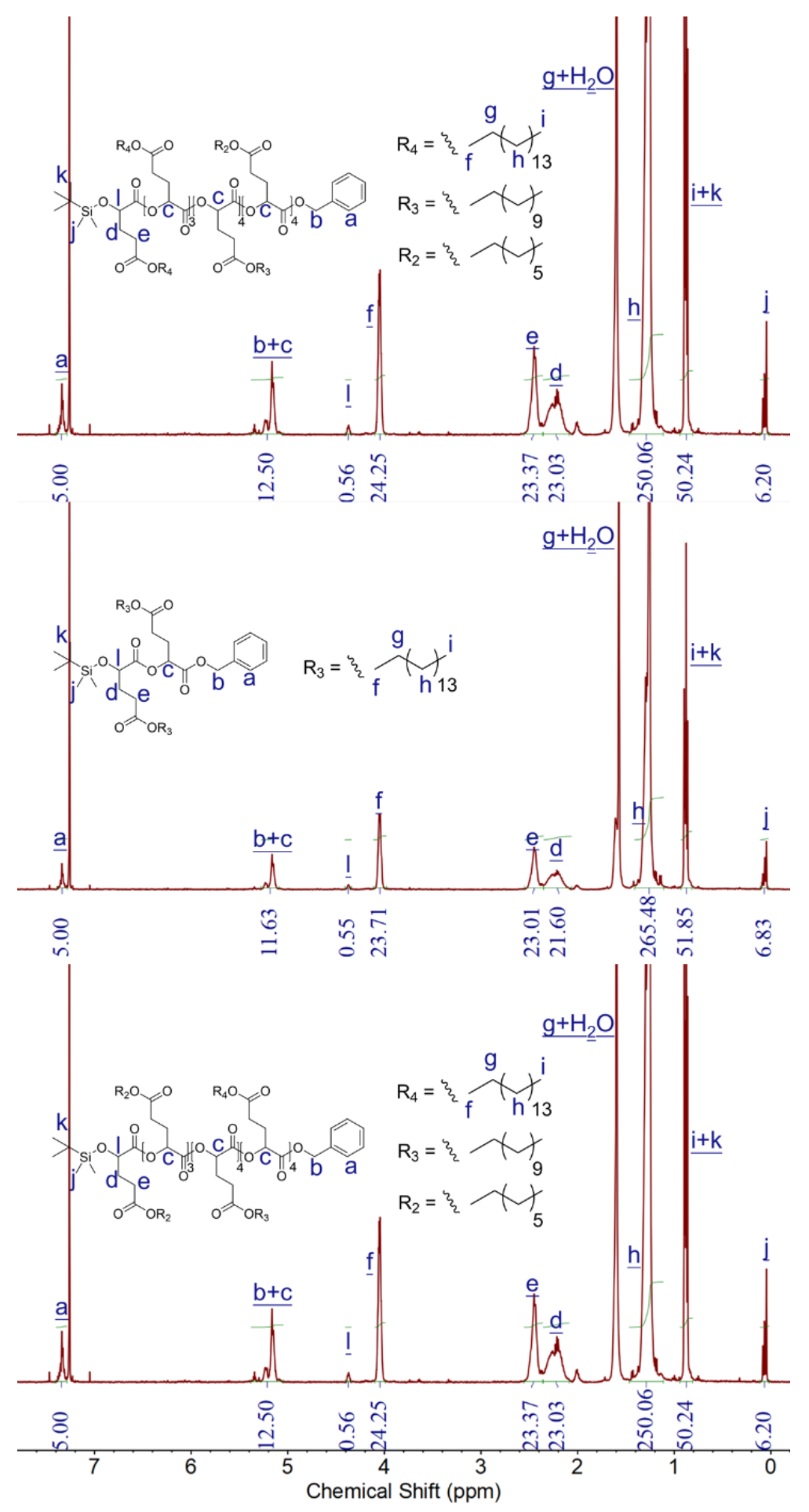

Figure S14. ${ }^{1} \mathrm{H}$ NMR spectra of discrete polymers $\mathrm{H}_{4} \mathrm{D}_{4} \mathrm{O}_{4}, \mathrm{D}_{12}$, and $\mathrm{O}_{4} \mathrm{D}_{4} \mathrm{H}_{4}$ (from top to bottom). 


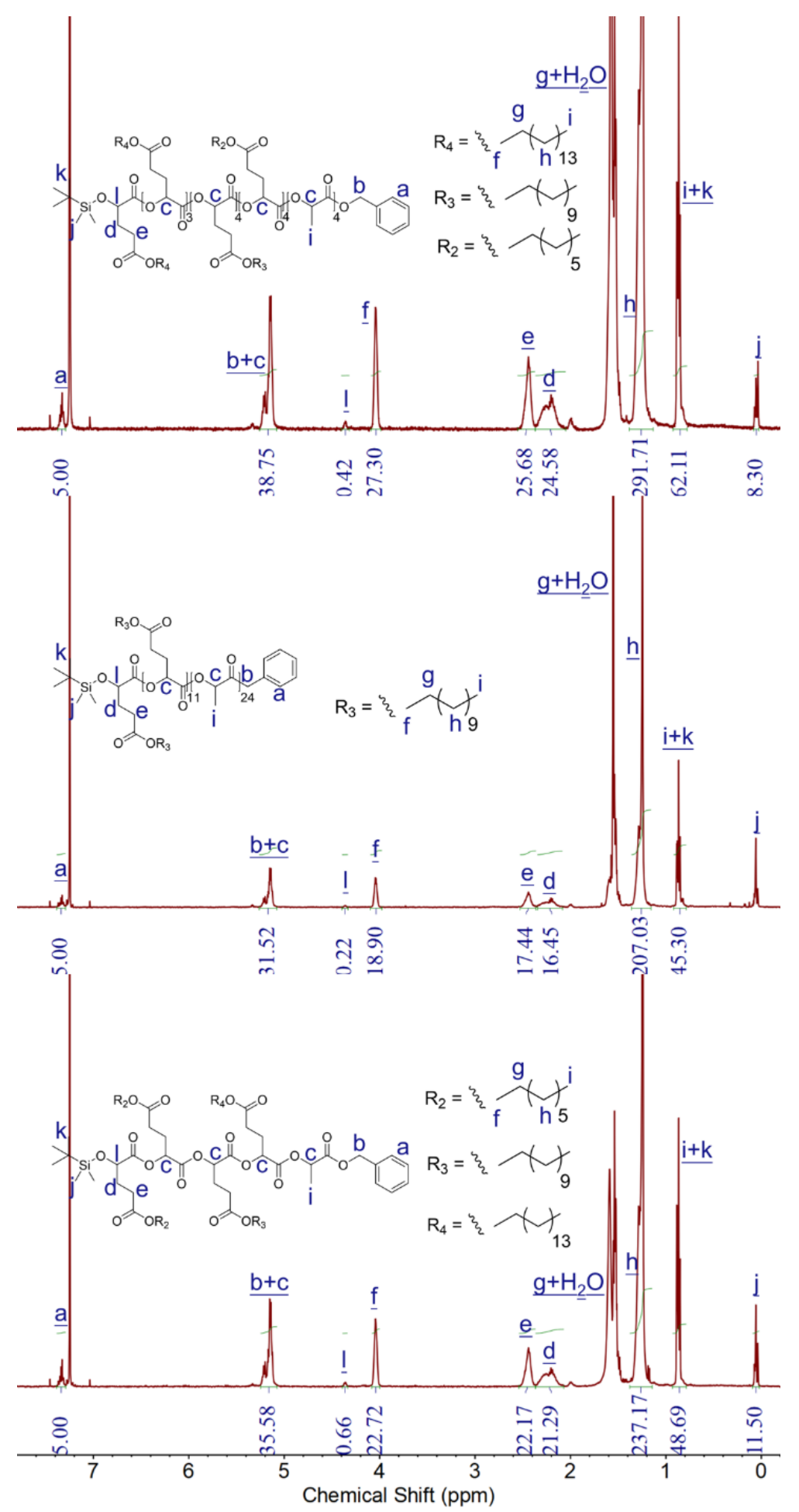

Figure S15. ${ }^{1} \mathrm{H}$ NMR spectra of discrete block copolymers $\mathrm{H}_{4} \mathrm{D}_{4} \mathrm{O}_{4}-\mathrm{L}_{24}, \mathrm{D}_{12}-\mathrm{L}_{24}$, and $\mathrm{O}_{4} \mathrm{D}_{4} \mathrm{H}_{4}-\mathrm{L}_{24}$ (from top to bottom). 

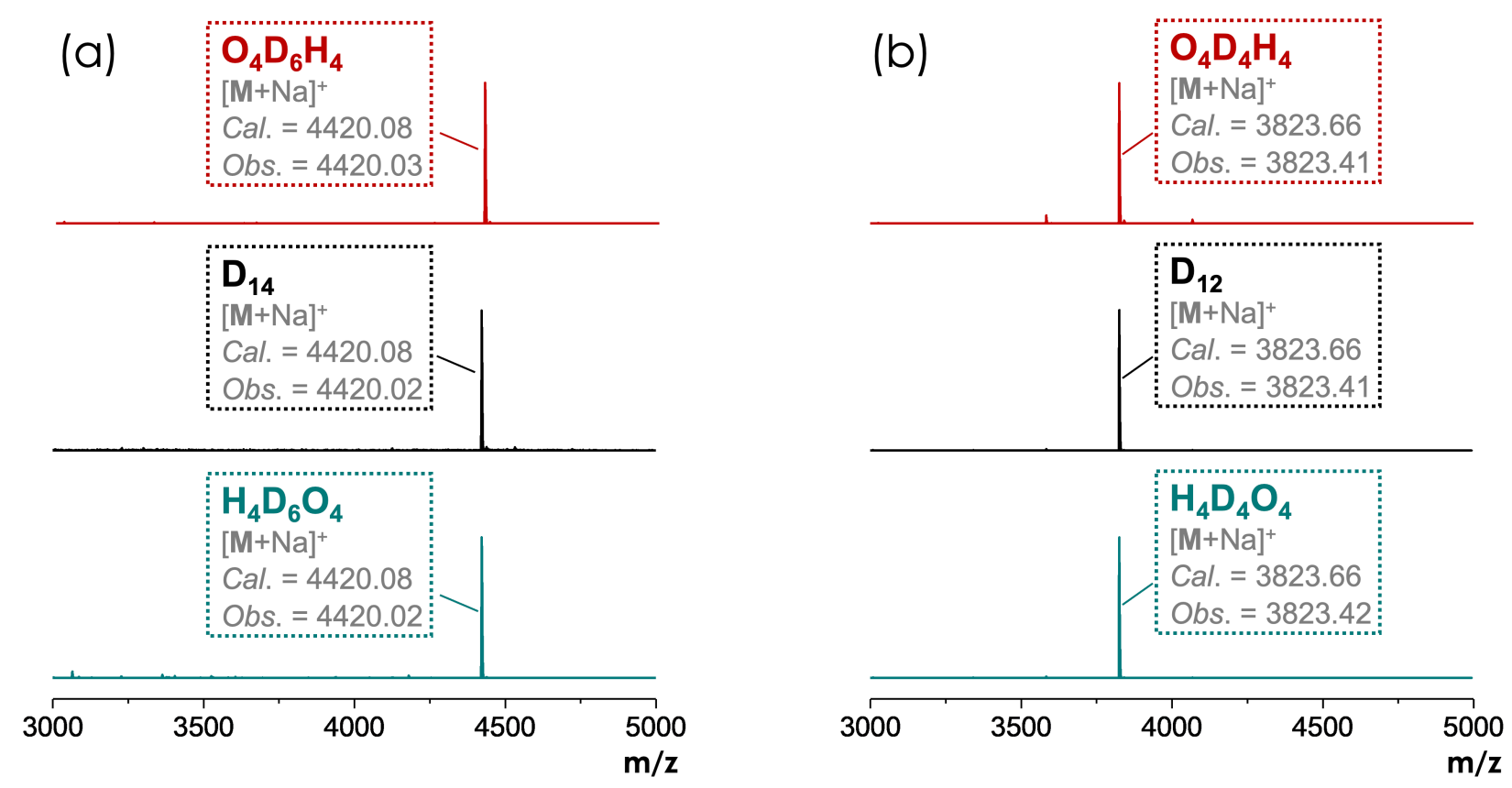

Figure S16. MALDI-ToF MS of $\mathrm{D}_{14}$ series (a) and $\mathrm{D}_{12}$ series (b).
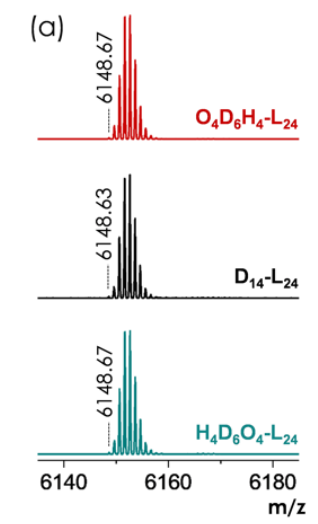

(e)
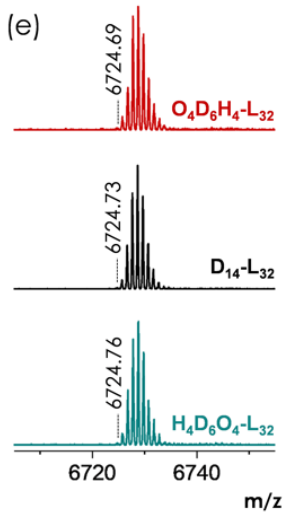
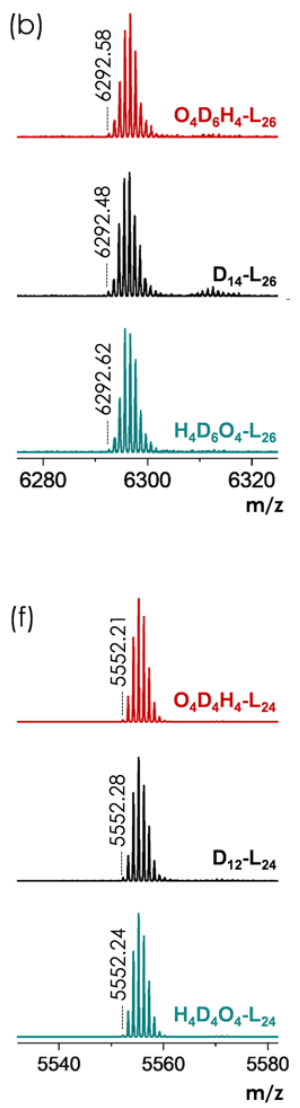

(c)
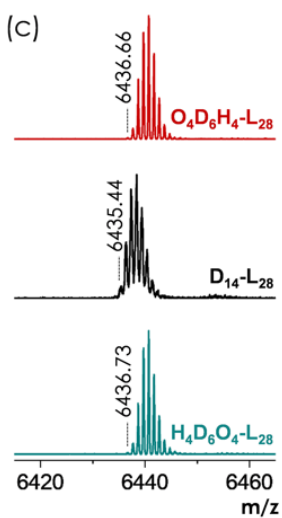

(g)
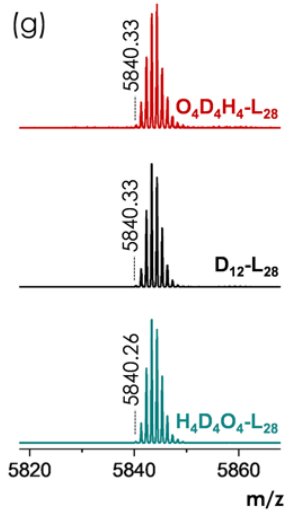

(d)
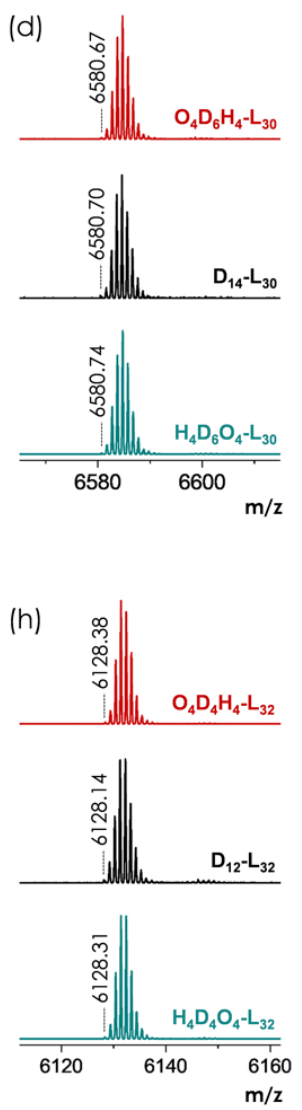

Figure S17. Enlarged MALDI-ToF MS of discrete block copolymers $\mathrm{D}_{14}-\mathrm{L}_{m}$ (a-e, black) and $\mathrm{D}_{12}-\mathrm{L}_{m}$ (f-h, black), and the corresponding isomers (dark red and dark cyan). Detailed sample information is listed in the figure. 

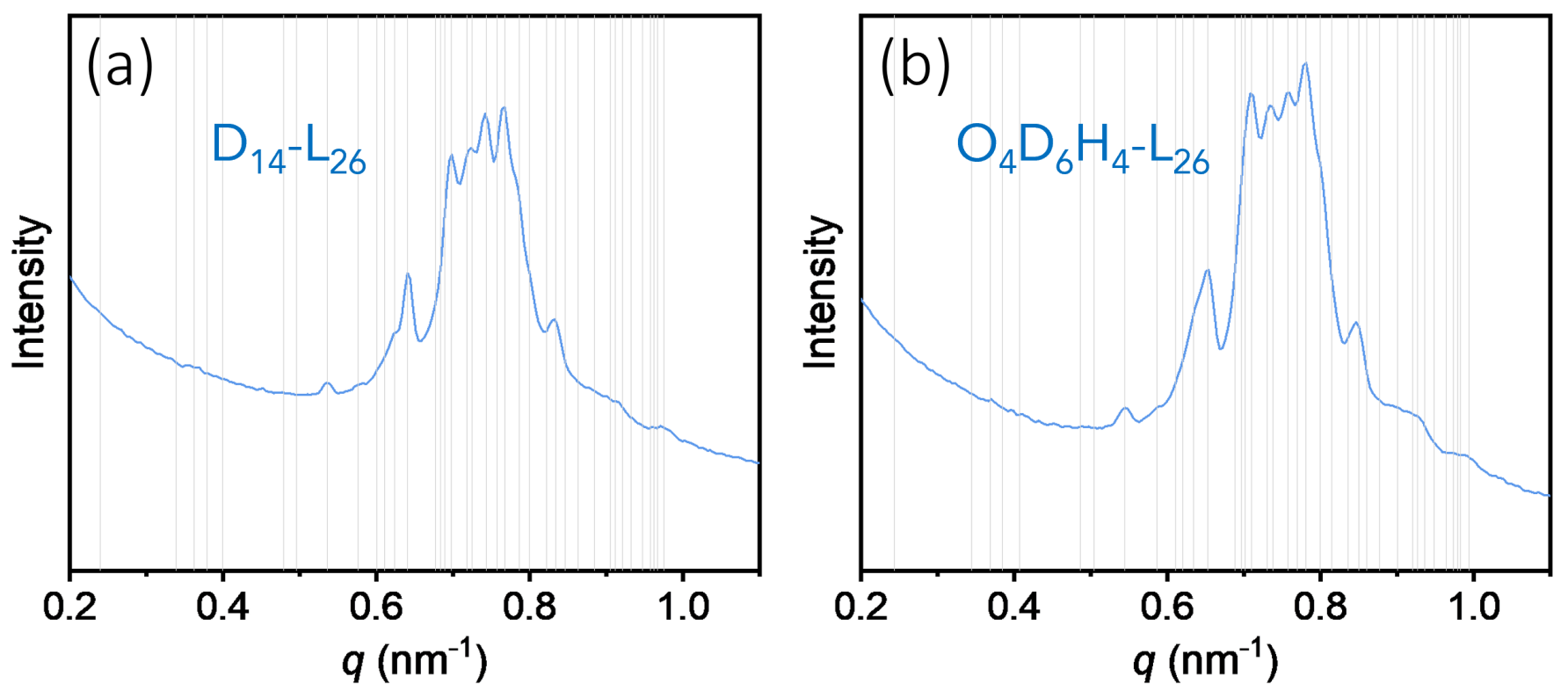

Figure S18. Peak indexing of SAXS profiles of $\mathrm{D}_{14}-\mathrm{L}_{26}$ (a) and $\mathrm{O}_{4} \mathrm{D}_{6} \mathrm{H}_{4}-\mathrm{L}_{28}$ (b). Droplines are the allowed reflections based on the calculated lattice parameter.

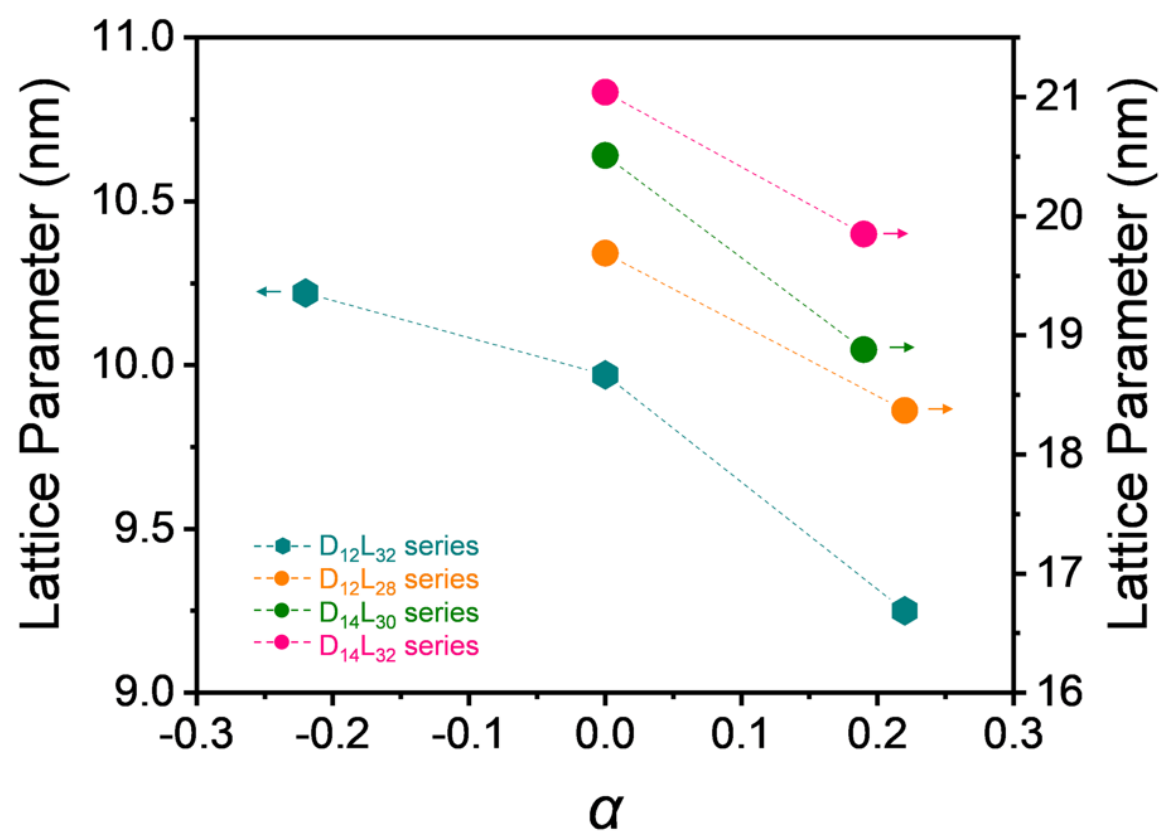

Figure S19. Variation of lattice dimensions as a function of $\alpha$. Solid circles represent A15 phase while hexagons represent HEX phase. Block copolymer series are labeled by different colors. Data are adopted from Table 2. 


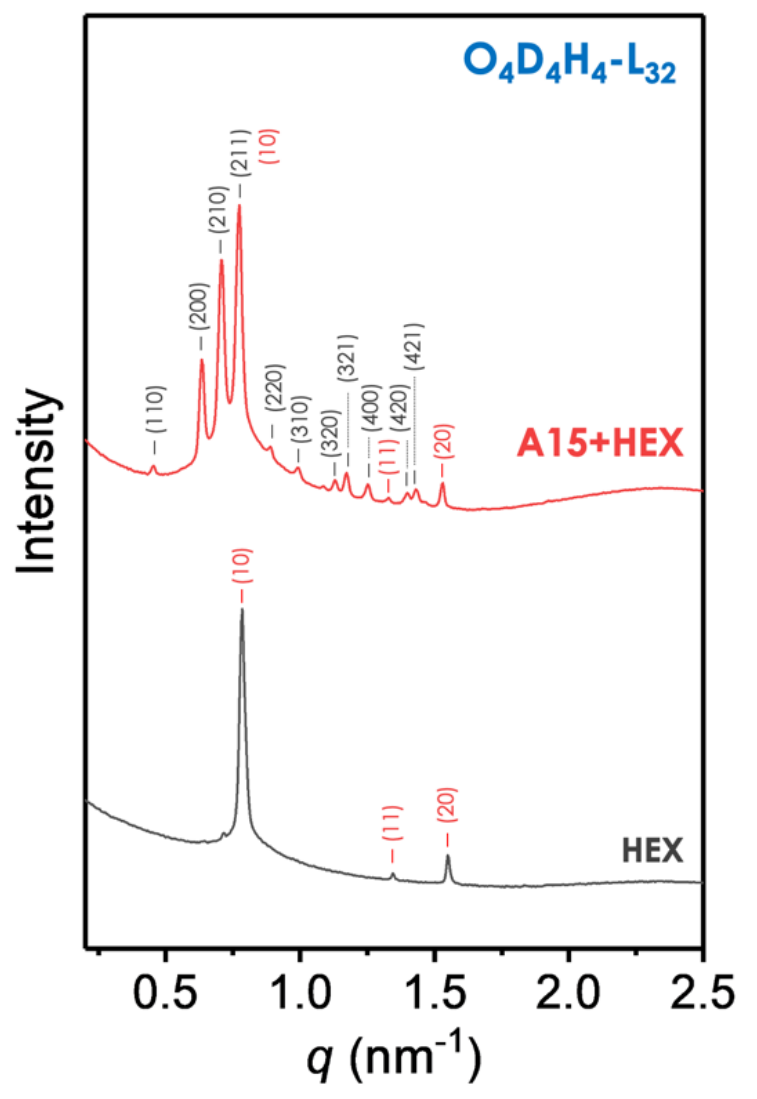

Figure S20. SAXS profiles of sample $\mathrm{O}_{4} \mathrm{D}_{4} \mathrm{H}_{4}-\mathrm{L}_{32}$ annealed at 35 (black) and $40{ }^{\circ} \mathrm{C}$ (red).
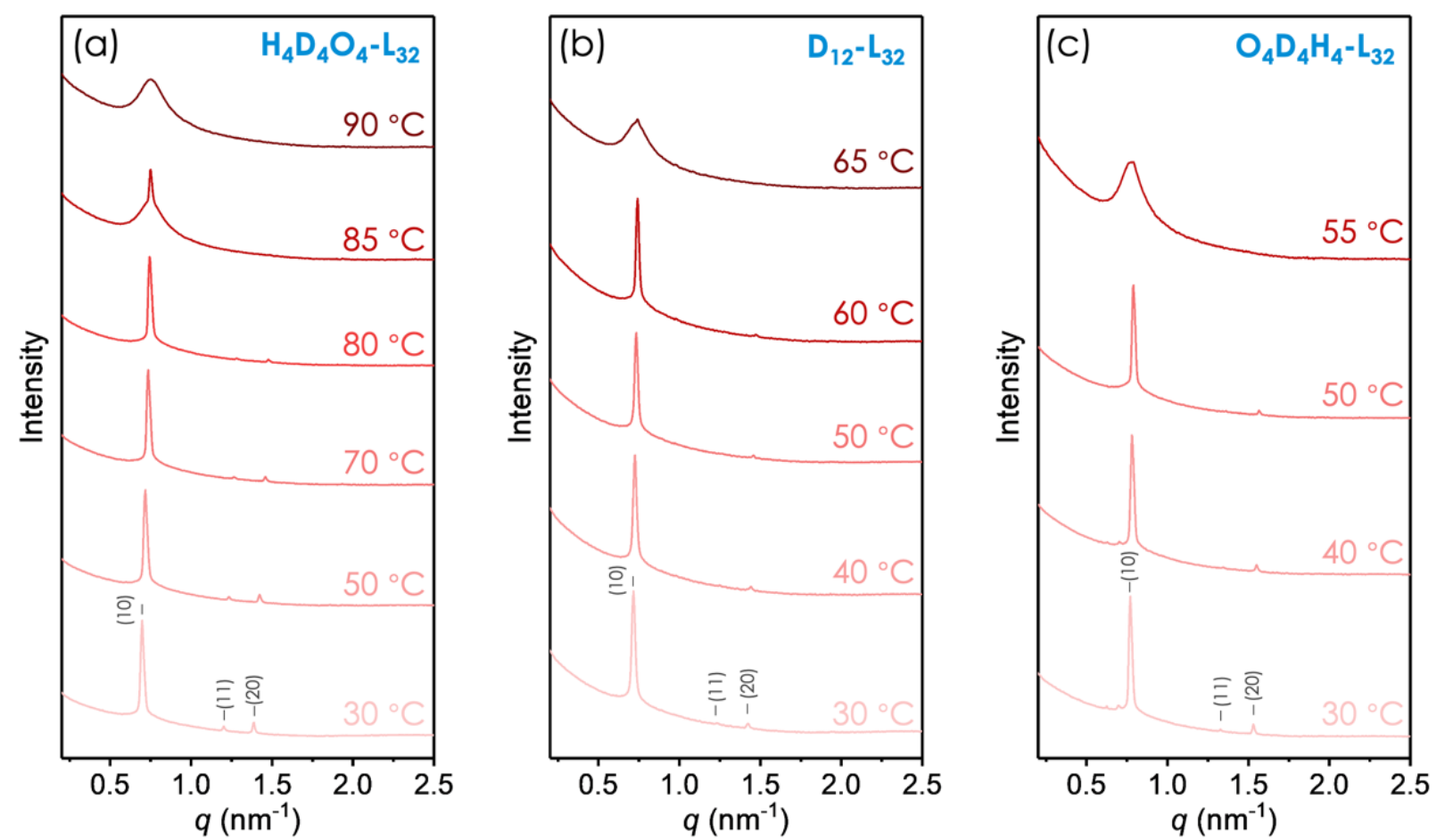

Figure S21. Temperature dependent SAXS profiles of $\mathrm{H}_{4} \mathrm{D}_{4} \mathrm{O}_{4}-\mathrm{L}_{32}(\mathrm{a}), \mathrm{D}_{12}-\mathrm{L}_{32}$ (b), and $\mathrm{O}_{4} \mathrm{D}_{4} \mathrm{H}_{4}-\mathrm{L}_{32}$ (c) (heating rate $\left.10{ }^{\circ} \mathrm{C} / \mathrm{min}\right)$. 


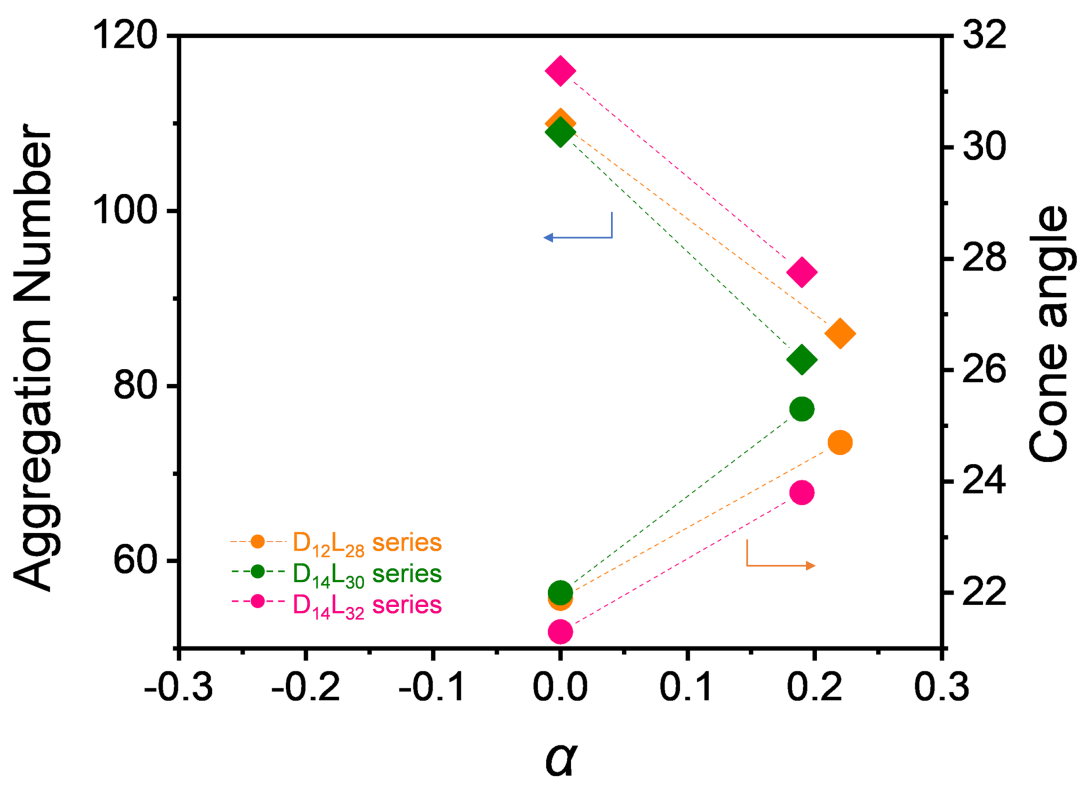

Figure S22. Variation of aggregation number (solid diamond) and cone angle (solid circle) as a function of $\alpha$. Block copolymer series are labeled by different colors. Data are adopted from Table 2.

\section{References}

(1) Tan, R.; Zhou, D.; Liu, B.; Sun, Y.; Liu, X.; Ma, Z.; Kong, D.; He, J.; Zhang, Z.; Dong, X.-H. Precise Modulation of Molecular Weight Distribution for Structural Engineering. Chem Sci 2019, 10, 10698-10705.

(2) Sun, Y.; Tan, R.; Ma, Z.; Zhou, D.; Li, J.; Kong, D.; and Dong, X.-H. Quantify the contribution of chain length heterogeneity on block copolymer self-assembly. Giant 2020, 4, 100037. 